\title{
The nature of the Class I population in Ophiuchus as revealed through gas and dust mapping
}

\author{
T. A. van Kempen ${ }^{1,2}$, E. F. van Dishoeck ${ }^{1,3}$, D. M. Salter ${ }^{1}$, M. R. Hogerheijde ${ }^{1}$, J. K. Jørgensen ${ }^{4}$, and A. C. A. Boogert ${ }^{5}$ \\ 1 Leiden University, Leiden Observatory, PO Box 9513, 2300 RA Leiden, The Netherlands \\ 2 Center for Astrophysics, 60 Garden Street, Cambridge, MA 02138, USA \\ e-mail: tvankempen@cfa.harvard.edu \\ 3 Max-Planck Institut für Extraterrestrische Physik (MPE), Giessenbachstr. 1, 85748 Garching, Germany \\ 4 Argelander-Institut für Astronomie, University of Bonn, Auf dem Hügel 71, 53121 Bonn, Germany \\ 5 Infrared Processing and Analysis Center (IPAC), NASA Herschel Science Center, Mail Code 100-22, California Institute of \\ Technology, Pasadena, CA 91125, USA
}

Received 23 June 2008 / Accepted 22 February 2009

\section{ABSTRACT}

\begin{abstract}
Context. The Ophiuchus clouds, in particular L 1688, are an excellent region to study the embedded phases of star formation, due to the relatively large number of protostars. However, the standard method of finding and characterizing embedded young stellar objects (YSOs) through just their infrared spectral slope does not yield a reliable sample. This may affect the age determinations, often derived from the statistics on the total number of embedded YSOs and pre-main sequence stars within a cloud.

Aims. Our aim is to characterize the structure of protostellar envelopes on an individual basis and to correctly identify the embedded YSO population of L 1688.

Methods. Spectral maps of the $\mathrm{HCO}^{+} J=4-3$ and $\mathrm{C}^{18} \mathrm{O} J=3-2$ lines, using the HARP-B array on the James Clerk Maxwell Telescope and SCUBA $850 \mu \mathrm{m}$ dust maps, are obtained of all sources in the L 1688 region with infrared spectral slopes consistent with, or close to, that of embedded YSOs. Selected $350 \mu \mathrm{m}$ maps obtained with the Caltech Submillimeter Observatory are presented as well. The properties, extent and variation of dense gas, column density and dust up to $1^{\prime}(\sim 7500 \mathrm{AU})$ are probed at $15^{\prime \prime}$ resolution. Using the spatial variation of the gas and dust, together with the intensity of the $\mathrm{HCO}^{+} J=4-3$ line, we are able to accurately identify the truly embedded YSOs and determine their properties.

Results. The protostellar envelopes range from 0.05 to $0.5 M_{\odot}$ in mass. The concentration of $\mathrm{HCO}^{+}$emission $(\sim 0.5$ to 0.9$)$ is generally higher than that of the dust concentration. Combined with absolute intensities, $\mathrm{HCO}^{+}$proves to be a better tracer of protostellar envelopes than dust, which can contain disk and cloud contributions. Our total sample of 45 sources, including all previously classified Class I sources, several flat-spectrum sources and some known disks, was re-classified using the molecular emission. Of these, only 17 sources are definitely embedded YSOs. Four of these embedded YSOs have little $\left(0.1-0.2 M_{\odot}\right)$ envelope material remaining and are likely at the interesting transitional stage from embedded YSO to T Tauri star. About half of the flat-spectrum sources are found to be embedded YSOs and about half are disks.

Conclusions. The presented classification method is successful in separating embedded YSOs from edge-on disks and confused sources. The total embedded population of the Ophiuchus L 1688 cloud is found almost exclusively in Oph-A, Oph-B2 and the Ophiuchus ridge with only three embedded YSOs not related to these regions. The detailed characterization presented will be necessary to interpret deep interferometric ALMA and future Herschel observations.
\end{abstract}

Key words. astrochemistry - circumstellar matter - stars: formation - submillimeter - ISM: clouds - ISM: molecules

\section{Introduction}

Low-mass young stellar objects (YSOs) have traditionally been classified using their observed infrared (IR) spectral slope, $\alpha_{\mathrm{IR}}$, from 2 to $\sim 20 \mu \mathrm{m}$ (Lada \& Wilking 1984; Adams et al. 1987) or their bolometric temperature, $T_{\text {bol }}$ (Myers \& Ladd 1993). Together with the subsequent discovery of the Class 0 stage (André et al. 1993) this led Greene et al. (1994) to identify 5 classes of YSOs:

- Class 0, no $\alpha_{2-14}$, high $L_{\text {sub-mm }} / L_{\text {bol }}$.

- Class I, $\alpha_{2-14}>0.3, T_{\text {bol }}<650 \mathrm{~K}$.

- Flat Spectrum, $-0.3<\alpha_{2-14}<0.3, T_{\text {bol }} \sim 400-800 \mathrm{~K}$.

- Class II, $-2<\alpha_{2-14}<-0.3,650<T_{\text {bol }}<2800 \mathrm{~K}$.

- Class III, $\alpha_{2-14}<-2, T_{\text {bol }}>2800 \mathrm{~K}$.

Each class is thought to be represent a different category, and probably evolutionary stage, of YSOs. Class 0 sources are the earliest, deeply embedded YSO stage; Class I sources are thought to be more evolved embedded YSOs, Class II the T Tauri stars with gas-rich circumstellar disks and Class III the pre-main sequence stars surrounded by tenuous or debris disks. Deep midIR photometry introduced the "flat-spectrum" (FS) sources (e.g. Greene et al. 1994), with IR spectral slope close to 0 and which may represent a stage intermediate between Class I and II. An accurate classification and physical characterization of YSOs is important for constraining the time scales of each of the phases and for determining the processes through which an object transitions from one phase to the next. In this study, we focus on the embedded YSO population and their transition to the T Tauri phase.

The $\rho$ Ophiuchus molecular clouds, part of the Gould Belt, are some of the nearest star-forming regions and contain many Class I and II sources. Consisting of two main clouds, L 1688 and L 1689, the star formation history and protostellar 
population of these regions have been studied extensively. Although the distance to Ophiuchus has long been debated (Knude \& Høg 1998), recent work constrains it to $120 \pm 4$ pc for L 1688 (Loinard et al. 2008). The large-scale structure of the Ophiuchus clouds at millimeter wavelengths was first mapped by Loren (1989) using the ${ }^{13} \mathrm{CO}$ molecular line emission with $2.4^{\prime}$ resolution. It was found that much of the cloud is in filamentary structures, but also that a diffuse foreground layer is present in Ophiuchus, resulting in a higher average extinction toward YSOs than in other clouds such as Taurus (Dickman \& Herbst 1990). Subsequent continuum observations at millimeter (mm) wavelengths mapped most of the large-scale structure present in detail, distinguishing the Oph-A through Oph-F regions within L 1688 (Mezger et al. 1992; Motte et al. 1998; Johnstone et al. 2000; Stanke et al. 2006; Young et al. 2006).

The YSO population was first identified by Elias (1978); Wilking \& Lada (1983); Wilking et al. (1989); Comeron et al. (1993) and Greene et al. (1994) using IR observations. With the arrival of (sub-)mm telescopes, VLA 1623 in L 1688 was identified as the first deeply embedded YSO (Wootten 1989; Loren et al. 1990; André et al. 1993). In more recent years, a large population of Class I and Class II sources has been found based on their IR spectral slopes, using space-based observatories such as the Infrared Space Observatory (ISO) (e.g. Liseau et al. 1999; Bontemps et al. 2001) and ground-based IR (e.g. Barsony et al. 1997, 2005). Most embedded sources in L 1688 are clustered around the filaments of the Oph-A, Oph-B2, Oph-E and $\mathrm{Oph}-\mathrm{F}$ regions, while the $\mathrm{Oph}-\mathrm{C}$ region only shows a single embedded source and Oph-D does not have any embedded YSO (Motte et al. 1998). In Oph-E and Oph-F most sources are lined up along a relatively small filament of material: for the purpose of this paper, we will adopt the name "Ophiuchus ridge" for this region.

With the launch of the Spitzer Space Telescope, the Ophiuchus cloud was included in the guaranteed time (GTO) and the "cores to disks" (c2d) Legacy program (Evans et al. 2003). Padgett et al. (2008) report on the results at 24, 70 and $160 \mu \mathrm{m}$ using the MIPS instrument, revealing the emission from the large-scale structure at mid and far-IR wavelengths. Jørgensen et al. (2008) compared the results from the c2d program with the COMPLETE $850 \mu \mathrm{m}$ SCUBA sub-millimeter dust mapping from Johnstone et al. (2000) and Ridge et al. (2006) to determine the association of YSOs with dense cores.

The stellar ages of the Class II and III sources in Ophiuchus were found to be $0.1-1$ Myr based on stellar spectroscopy compared with evolutionary tracks, indicating a relatively young age for the total cloud (Greene \& Meyer 1995; Luhman \& Rieke 1999). The Star-Formation Efficiency (SFE) was recently calculated with Spitzer and SCUBA photometry to be of the order of $13 \%$ within the cores and $4 \%$ in the cloud as found by Evans et al. (2009) and Jørgensen et al. (2008), lower than previous determinations (Wilking \& Lada 1983).

The relative timescales of the different phases are determined by the number of objects in each class of YSOs (e.g. Evans et al. 2009). Recent high resolution ground-based (near)IR imaging show that some of the Class I sources in Ophiuchus are physically different from an embedded YSO, confusing these timescales determinations. For example, the Class I source CRBR 2422.8-3423 was found to be an extincted edge-on disk from near-IR imaging (Brandner et al. 2000; Pontoppidan et al. 2005). The source OphE MM3, classified as a starless core by Motte et al. (1998), was also shown to be a edge-on disk in the same study (Brandner et al. 2000). The source IRS 46 has no associated protostellar envelope and was re-classified based on
Spitzer and sub-mm data as a disk (Lahuis et al. 2006). Much of the reddening seen in the IR originates from the nearby envelope associated with IRS 44.

Foreground material can also heavily influence the identification and subsequent analysis of embedded sources (e.g., Luhman \& Rieke 1999). An excellent example is provided by the Class I source Elias 29 in L 1688, which has two foreground layers in addition to the ridge of material in which the YSO is embedded (Boogert et al. 2002). Only a combination of molecular line emission at sub-millimeter and IR spectroscopy could constrain the protostellar envelope as well as the immediate environment around it (Boogert et al. 2000, 2002). Indeed, IR spectroscopy can be used as a complementary diagnostic and spectra of many of the YSOs in Ophiuchus have been taken, using ISO, Spitzer or groud-based telescopes (e.g. Alexander et al. 2003; Pontoppidan et al. 2003; Boogert et al. 2008). Ice absorption features such as the $3 \mu \mathrm{m} \mathrm{H}_{2} \mathrm{O}$ and $15.2 \mu \mathrm{m} \mathrm{CO}_{2}$ bands are usually associated with embedded sources whereas silicate emission at 10 and $20 \mu \mathrm{m}$ is characteristic of Class II sources, but foreground absorption and edge-on disks can confuse this classification (Boogert et al. 2002; Pontoppidan et al. 2005).

In recent years, several detailed modelling efforts have been carried out to study the relations between the observed spectral energy distribution (SED) and the physical structure of embedded YSOs (e.g. Jørgensen et al. 2002; Whitney et al. 2003b; Schöier et al. 2004; Young et al. 2004; Robitaille et al. 2006, 2007; Crapsi et al. 2008). Whitney et al. (2003b) show that it is possible for embedded YSOs with a face-on projection to be classified as Class II. Due to their orientation, these sources are viewed straight down the outflow cone, directly onto the central star and disk system. Crapsi et al. (2008) show that a significant fraction of the Class I sources may be edge-on flaring disks, that have already lost their protostellar envelope. The spectral slope is much steeper than expected due to their structure.

A prime characteristic and component of embedded YSOs is the presence of dense centrally condensed envelopes. While dust maps at sub-millimeter wavelengths have become very popular to trace the early stages of star formation (e.g. Motte et al. 1998; Shirley et al. 2000; Johnstone et al. 2000; Stanke et al. 2006), the continuum emission at these wavelengths is dominated by the cold outer envelope and cloud material, with disks starting to contribute as the envelope disperses (e.g. Hogerheijde et al. 1997; Looney et al. 2000; Young et al. 2003). Singledish dust continuum data by themselves are not able to distinguish between dense cores and envelope or foreground material, nor quantify any disk contributions. However, the dense gas $\left(\sim 10^{6} \mathrm{~cm}^{-3}\right)$ located in the inner regions of protostellar envelopes is uniquely probed by molecular lines with high critical densities at sub-millimeter wavelengths. Observations of deeply embedded Class 0 YSOs have indeed revealed strong sub-millimeter lines of various molecules (e.g. Blake et al. 1994, 1995; Schöier et al. 2002; Jørgensen 2004; Maret et al. 2004, 2005), but only a few studies have been carried out on more evolved Class I embedded YSOs (e.g. Hogerheijde et al. 1997).

A good high density tracer is the $\mathrm{HCO}^{+}$molecule, for which the $J=4-3$ line both has a high critical density of $>10^{6} \mathrm{~cm}^{-3}$ and is accessible from the ground. Dense gas is also found in the circumstellar disk on scales of a few tens to hundreds AU, but such regions are generally diluted by an order of magnitude in single-dish observations. In contrast, molecular lines with much lower critical densities, such as the low excitation $\mathrm{C}^{18} \mathrm{O}$ transitions, contain much higher contributions from low density material. This makes these lines well-suited as column density tracers for large-scale cloud material and the cold outer regions of 
the protostellar envelope (e.g. Jørgensen et al. 2002). Both the $\mathrm{HCO}^{+}$and $\mathrm{C}^{18} \mathrm{O}$ data have velocity resolutions of $0.1 \mathrm{~km} \mathrm{~s}^{-1}$ or better, thus allowing foreground clouds to be identified.

Most sub-millimeter line data so far have been single pixel spectra toward the YSO with at best a few positions around specific YSOs. The recently commissioned HARP-B instrument is a 16-pixel receiver, operating in the 320 to $370 \mathrm{GHz}$ atmospheric window allowing rapid mapping of small $\left(2^{\prime}\right)$ regions (Smith et al. 2003). HARP-B is mounted on the James Clerk Maxwell Telescope (JCMT) ${ }^{1}$.

We present here HARP-B maps of all Class I sources in the $\mathrm{L} 1688$ region in $\mathrm{C}^{18} \mathrm{O} 3-2$ and $\mathrm{HCO}^{+} 4-3$. The combination of these two molecular lines allows us to differentiate between protostellar envelopes, dense cores and foreground cloud material, as well as edge-on disks. The goal of this paper is to characterize the envelopes of the embedded source population of L 1688 , as well as present a new method for identifying truly embedded sources and separate them from (obscured) edge-on disks using the dense gas present in embedded YSOs. In Sect. 2, a sample of Class I sources in the L 1688 core is selected. In Sect. 3, we discuss the details of the heterodyne observations carried out at the JCMT and the Atacama Pathfinder EXperiment (APEX) ${ }^{2}$, as well as the supplementary observations obtained in a continuum i.e. wideband mode. Section 4 presents the maps and spectra and in Sect. 5 we analyze the properties of the gas and dust of the sources in the sample. The environment around the YSOs, column density, envelope gas and concentration of the $\mathrm{HCO}^{+}$are discussed. In Sect. 6, we present a new method for identifying embedded YSOs from (edge-on) disks and apply this method to the sample. This classification is then compared to traditional methods as well as other recently proposed methods. The main conclusions of the paper are given in Sect. 7.

\section{Sample selection}

Of the known (embedded) YSO population within L 1688, 45 objects were selected for our sample using several criteria. First, we require all sources be located within the $850 \mu \mathrm{m}$ dust continuum map made by the COMPLETE project of L 1688 using SCUBA on the JCMT (Johnstone et al. 2000).

Second, sources must be included in the area covered by the c2d program using IRAC and MIPS on Spitzer (Evans et al. 2003; Padgett et al. 2008). All sources classified as Class I in either André \& Montmerle (1994), Barsony et al. (1997), Bontemps et al. (2001) or the c2d delivery document (Evans et al. 2007) $)^{3}$ are included with luminosities $>0.04 L_{\odot}$. Although these 41 objects have been classified as Class I in one or more of these papers, only 6 of these have been classified consistently as Class I in all studies. Most other sources are classified as either Class II or Flat spectrum sources at least once. All such sources are included in the analysis of this paper, but conversely, our sample does not include all Flat spectrum or Class II sources listed in the c2d survey. Known edge-on disks such as 2MASS 16282, IRS 46, OphE MM3 and CRBR 2422.2-3423,

\footnotetext{
1 The James Clerk Maxwell Telescope is operated by The Joint Astronomy Centre on behalf of the Science and Technology Facilities Council of the United Kingdom, the Netherlands Organisation for Scientific Research, and the National Research Council of Canada.

2 This publication is based on data acquired with the Atacama Pathfinder Experiment (APEX). APEX is a collaboration between the Max-Planck-Institut fur Radioastronomie, the European Southern Observatory, and the Onsala Space Observatory.

${ }^{3}$ http://ssc.spitzer.caltech.edu/
}

are among these 41 sources and are included within the sample to illustrate the results of our method for such sources. The sample should not contain any reddened background main-sequence stars which are readily identified in the $\mathrm{c} 2 \mathrm{~d}$ analysis. However, other background sources, such as AGB stars or background infrared galaxies, may be present.

Four sources with $\alpha_{2-24 \mu \mathrm{m}}>0.0$ were found only in the recent Spitzer observations. These are SSTc2d J162527.6243648, SSTc2d J162741.6-244645, SSTc2d J162748.2-244225 and SSTc2d J162857.9-24405. The names C2D-162527.6, C2D-162741.6, C2D-162748.2 and C2D-162857.9 are adopted. VLA 1623-2418 is included as an embedded Class 0, but it is generally absent from the above studies. In addition, IRS 63 was included, although it is not part of the L 1688 core, due to its interesting characteristics as a Class I source as observed with the SubMillimeter Array (SMA). These recent interferometric results suggest that this source is an embedded YSO with little envelope material left, and, as such, presents an interesting test case for the proposed method of identifying truly embedded sources (Lommen et al. 2008). Four disk sources within or near to the L 1688 region, Haro 1-4, DoAr 25, SR 9 and SR 21, were included to serve as a sample of known disk sources.

The final source sample, which covers all potential Class I sources, can be found in Table 1. The positions as found in Bontemps et al. (2001) were used where available. If the source was not included in Bontemps et al. (2001), or if confusion exists due to nearby IR sources, the position as found in the c2d delivery document (Evans et al. 2007) was adopted. For sources in common, most positions agree within $3^{\prime \prime}$. Figure 1 shows the distribution of the sample as plotted on the SCUBA $850 \mu \mathrm{m}$ map of L 1688 (Johnstone et al. 2000; Ridge et al. 2006; Di Francesco et al. 2008).

The table includes the spectral index $\alpha_{2-24} \mu \mathrm{m}$ given in Evans et al. (2009) calculated from the 2MASS, IRAC and MIPS (24 $\mu \mathrm{m}$ only) fluxes. No $\alpha_{\mathrm{IR}}$ could be determined from Spitzer data for VLA 1623 (too faint).

\section{Observations}

\subsection{Gas line maps}

The majority of the sample was observed in $\mathrm{HCO}^{+}$4-3 $(356.7341 \mathrm{GHz})$ and $\mathrm{C}^{18} \mathrm{O} 3-2(329.3305 \mathrm{GHz})$ using the recently commissioned 16-pixel heterodyne array receiver HARP-B on the James Clerk Maxwell Telescope (JCMT). The high spectral resolution mode of $0.05 \mathrm{~km} \mathrm{~s}^{-1}$ available with the ACSIS back-end was used to disentangle foreground material as well as any contributions from outflowing material. Spectra were subsequently binned to $0.15 \mathrm{~km} \mathrm{~s}^{-1}$.

HARP-B observations of 30 sources in 21 fields were carried out during July and August 2007 in $\mathrm{C}^{18} \mathrm{O} 3-2$ and $\mathrm{HCO}^{+} 4-3$ under weather conditions with an atmospheric optical depth, $\tau_{225 \mathrm{GHz}}$ ranging from 0.035 to 0.08 (precipitable water vapor of 0.7 to $1.6 \mathrm{~mm}$ ). The fields were observed down to a rms noise of $0.1 \mathrm{~K}$ in a $0.5 \mathrm{~km} \mathrm{~s}^{-1}$ bin. The HARP-B pixels have typical single side-band system temperatures of 300-350 K. The 16 receivers are arranged in a $4 \times 4$ pattern, separated by $30^{\prime \prime}$. This gives a total foot print of $2^{\prime}$ with a spatial resolution of $15^{\prime \prime}$, the beam of the JCMT at $345 \mathrm{GHz}$. The $2^{\prime} \times 2^{\prime}$ fields were mapped using the specifically designed jiggle mode HARP4 4 . A position switch of typically $30^{\prime}$ in azimuth was used, with larger throws if needed. The Class 0 source IRAS 16293-2422 was used as a line

\footnotetext{
${ }^{4}$ See JCMT website http : //www . jcmt . jach. hawai i . edu/
} 
Table 1. Sample of embedded sources in L 1688, with additional known disks included.

\begin{tabular}{|c|c|c|c|c|c|}
\hline \multirow[t]{2}{*}{ Source } & \multirow[t]{2}{*}{ Other names } & \multicolumn{2}{|c|}{ Coordinates (J2000) } & \multirow[t]{2}{*}{ Ref. $^{a}$} & \multirow[t]{2}{*}{$\alpha_{2-24 \mu \mathrm{m}^{b}}$} \\
\hline & & RA & Dec & & \\
\hline C2D-162527.6 & SSTc2d J162527.6-243648 & $16: 25: 27.6$ & $-24: 36: 48.4$ & 2 & 0.36 \\
\hline GSS 26 & & $16: 26: 10.4$ & $-24: 20: 58$ & 1 & -0.46 \\
\hline CRBR 2315.8-1700 & & $16: 26: 17.2$ & $-24: 23: 45.1$ & 2 & 0.69 \\
\hline CRBR 2317.3-1925.3 & SKS $1-10$ & $16: 26: 18.8$ & $-24: 26: 13$ & 1 & -0.56 \\
\hline VSSG 1 & Elias 20 & $16: 26: 18.9$ & $-24: 28: 22$ & 1 & -0.73 \\
\hline GSS 30 & Elias 21/GY 6/GSS 30-IRS1 & $16: 26: 21.4$ & $-24: 23: 04.1$ & 2 & 1.46 \\
\hline LFAM 1 & GSS 30-IRS3 & $16: 26: 21.7$ & -242251.4 & 2 & 0.73 \\
\hline CRBR 2324.1-1619 & & $16: 26: 25.5$ & $-24: 23: 01.6$ & 2 & 0.87 \\
\hline VLA 1623 & VLA $1623.4-2418$ & $16: 26: 26.4$ & $-24: 24: 30.3$ & 2 & no \\
\hline GY 51 & VSSG 27 & $16: 26: 30.5$ & $-24: 22: 59$ & 1 & 0.05 \\
\hline CRBR 2339.1-2032 & GY 91 & $16: 26: 40.5$ & $-24: 27: 14.3$ & 2 & 0.45 \\
\hline WL 12 & GY 111 & $16: 26: 44.0$ & $-24: 34: 48$ & 1 & 2.49 \\
\hline WL 2 & GY 128 & $16: 26: 48.6$ & $-24: 28: 39$ & 1 & 0.02 \\
\hline LFAM 26 & CRBR 2403.7/GY 197 & $16: 27: 05.3$ & $-24: 36: 29.8$ & 2 & 1.27 \\
\hline WL 17 & GY 205 & $16: 27: 07.0$ & $-24: 38: 16.0$ & 1 & 0.61 \\
\hline Elias 29 & WL15/GY 214 & $16: 27: 09.6$ & $-24: 37: 21.0$ & 1 & 1.69 \\
\hline GY 224 & & $16: 27: 11.4$ & $-24: 40: 46$ & 1 & -0.05 \\
\hline WL 19 & GY 227 & $16: 27: 11.9$ & $-24: 38: 31.0$ & 1 & -0.43 \\
\hline WL 20S & GY 240 & $16: 27: 15.9$ & $-24: 38: 46$ & 1 & 2.75 \\
\hline IRS 37 & GY 244 & $16: 27: 17.6$ & $-24: 28: 58$ & 1 & 0.25 \\
\hline WL 3 & GY 249 & $16: 27: 19.3$ & $-24: 28: 45$ & 1 & -0.03 \\
\hline IRS 42 & GY 252 & $16: 27: 21.6$ & $-24: 41: 42$ & 1 & -0.03 \\
\hline WL 6 & GY 254 & $16: 27: 21.8$ & $-24: 29: 55$ & 1 & 0.72 \\
\hline GY 256 & & $16: 27: 22.0$ & $-24: 29: 39.9$ & 2 & -0.05 \\
\hline IRS 43 & GY 265 & $16: 27: 27.1$ & $-24: 40: 51$ & 1 & 1.17 \\
\hline IRS 44 & GY 269 & $16: 27: 28.3$ & $-24: 39: 33.0$ & 1 & 2.29 \\
\hline Elias 32 & IRS 45/GY 273/VSSG 18 & $16: 27: 28.6$ & $-24: 27: 19.8$ & 2 & -0.03 \\
\hline Elias 33 & IRS 47/GY 279/VSSG 17 & $16: 27: 30.1$ & $-24: 27: 43$ & 1 & -0.12 \\
\hline IRS 48 & GY 304 & $16: 27: 37.2$ & $-24: 30: 34$ & 1 & 0.88 \\
\hline GY 312 & & $16: 27: 38.9$ & $-24: 40: 20.5$ & 2 & 0.64 \\
\hline IRS 51 & GY3 15 & $16: 27: 40.0$ & $-24: 43: 13$ & 1 & -0.15 \\
\hline C2D-162741.6 & SSTc2d J162741.6-244645 & $16: 27: 41.6$ & $-24: 46: 44.6$ & 2 & 0.32 \\
\hline C2D-162748.2 & SSTc2d J162748.2-244225 & $16: 27: 48.2$ & $-24: 42: 35.6$ & 2 & 1.55 \\
\hline IRS 54 & GY 378 & $16: 27: 51.7$ & $-24: 31: 46.0$ & 1 & 0.03 \\
\hline IRAS 16285-2355 & & $16: 28: 21.6$ & $-24: 36: 23.7$ & 2 & 1.23 \\
\hline C2D-162857.9 & SSTc2d J162857.9-244055 & $16: 28: 57.9$ & $-24: 40: 54.9$ & 2 & 0.67 \\
\hline IRS 63 & & $16: 31: 35.7$ & $-24: 01: 29.5$ & 2 & 0.14 \\
\hline & Known Disks & & & & \\
\hline Haro 1-4 & DoAr 16 & $16: 25: 10.5$ & $-23: 19: 14.5$ & 2 & -0.89 \\
\hline DoAR 25 & GY 17 & $16: 26: 24.0$ & $-24: 43: 09.0$ & 1 & -1.12 \\
\hline OphE MM3 & & $16: 27: 05.9$ & $-24: 37: 08.2$ & 2 & -0.33 \\
\hline SR 21 & Elias 30 & $16: 27: 10.2$ & $-24: 19: 16.0$ & 1 & -0.79 \\
\hline CRBR 2422.8-3423.8 & CRBR 85 & $16: 27: 24.8$ & $-24: 41: 03.0$ & 1 & 1.01 \\
\hline IRS 46 & GY 274 & $16: 27: 29.7$ & $-24: 39: 16.0$ & 1 & 0.18 \\
\hline SR 9 & IRS 52/Elias 34 & $16: 27: 40.5$ & $-24: 22: 07.0$ & 1 & -1.07 \\
\hline 2MASS 16282 & & $16: 28: 13.7$ & $-24: 31: 39.0$ & 2 & -1.55 \\
\hline
\end{tabular}

${ }^{a}$ References for positions: 1:Bontemps et al. (2001); 2: c2d delivery document.

${ }^{b}$ From Evans et al. (2009). See also Sect. 5.5.

calibrator and pointing source. Calibration errors are expected to dominate the flux uncertainties, and are estimated at $20 \%$. Pointing was checked every two hours and was generally found to be within $2-3^{\prime \prime}$. The map was re-sampled with a pixel size of $5^{\prime \prime}$, which is significantly larger than the pointing error. The main-beam efficiency was taken to be 0.67 . Data were reduced using the STARLINK package GAIA and the CLASS reduction package.

\subsection{Gas single pixel spectra}

Supplementary data of $\mathrm{HCO}^{+} 4-3$ were taken at the APEX telescope during July 2007, using the APEX-2a receiver. All sources were observed for which no HARP-B data were taken, except GSS 26 and GY 51. The APEX observations were done in excellent weather conditions with $\tau_{225 \mathrm{GHz}}$ ranging from 0.01 to 0.04 (PWV 0.2 to $0.8 \mathrm{~mm}$ ). Single spectra were taken with a spectral 


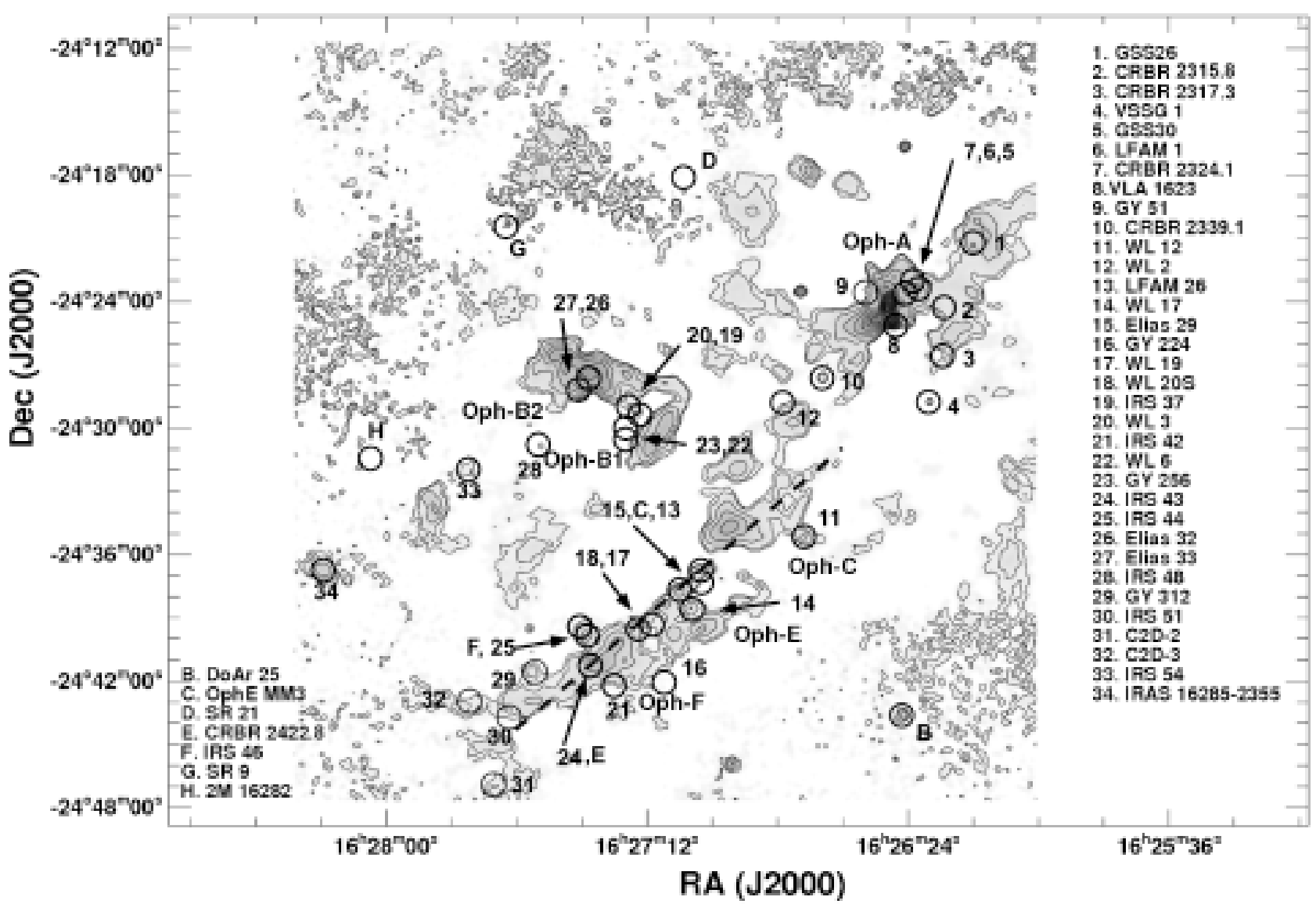

Fig. 1. The L 1688 core in Ophiuchus. In grey-scale and contours the $850 \mu \mathrm{m}$ SCUBA map as published by Johnstone et al. (2000) and Di Francesco et al. (2008) is shown. The locations of all the sources as observed in this study are shown, except for C2D-162527.6, Haro 1-4, C2D-162748.2 and IRS 63. C2D-16274.1 is marked as C2D-2 and C2D-162748.2 as C2D-3. The dashed line indicates the Oph ridge.

resolution of $0.4 \mathrm{~km} \mathrm{~s}^{-1}$ down to an rms of $0.3 \mathrm{~K}$. Calibration errors are estimated to be $\sim 20 \%$. The APEX $12-\mathrm{m}$ dish is slightly smaller than the JCMT 15-m dish, producing a beam of $18^{\prime \prime}$ instead of $15^{\prime \prime}$. Pointing errors are $<4^{\prime \prime}$. Beam efficiency is 0.70 .

For the sources GSS 30, VLA 1623, WL 20S, IRS 42, IRS 43, Elias 32 and Elias 33, $\mathrm{C}^{18} \mathrm{O} 3-2$ spectra were obtained from the CADC archive ${ }^{5}$. These spectra were taken with the $\mathrm{RxB}$ receiver during September 2005.

\subsection{Dust maps}

The $850 \mu \mathrm{m}$ continuum data of the Ophiuchus region, obtained within the scope of the COMPLETE project using the SCUBA instrument on the JCMT, were used to characterize the dust in the environments around all sources of the sample (Johnstone et al. 2000; Ridge et al. 2006; Di Francesco et al. 2008). The most recent map, version 3 , was used to extract the information (see Fig. 1). This version includes a correction for the chopped out-emission. Integrated fluxes within regions with radii ranging from $25^{\prime \prime}$ to $40^{\prime \prime}$ were extracted from the map (see Table 4). The exact radii were selected by calculating the FWHM to the peak flux of each source. Comparison with the map and published fluxes by Johnstone et al. (2000) within similar radii found by a clumpfind routine showed that fluxes agreed within $5 \%$, significantly better than the calibration error of $20 \%$

\footnotetext{
5 See

http://www. cadc-ccda.hia-iha.nrc-cnrc.gc.ca/jcmt/
}

for SCUBA. The sensitivity of the SCUBA map is such that sources down to $90 \mathrm{mJy}(3 \sigma)$ can be detected.

In addition, 11 sources were observed using the SHARC-II $350 \mu \mathrm{m}$ continuum instrument at the Caltech Submillimeter Observatory (CSO) ${ }^{6}$ during April 2003 (Dowell et al. 2003). The array has $12 \times 32$ pixels, spanning $4.85^{\prime \prime}$ per pixel, which results in a footprint of $1^{\prime} \times 2.6^{\prime}$ and a beam size of $9^{\prime \prime}$. The sources observed with the CSO were GSS 30, WL 12, Elias 29, VLA 1623, OphE MM3, WL 20S, WL 6, IRS 43, VSSG 17, IRS 51, 2MASS 16282. The data were reduced with the CRUSH package and calibrated using observations of Mars and Saturn. If no planets were available during the specific night, IRAS 162932422 was used to calibrate the data. The calibration errors are estimated to be on the order of $30-40 \%$ and assumed to dominate the error in the flux estimate over other instrumental errors or $S / N$ considerations. The sensivity of the SHARC-II maps is such that sources down to $400 \mathrm{mJy}(3 \sigma)$ can be detected. For a typical dust flux law $\propto v^{3.5}$, this implies that the SHARC-II data are a factor of 4 more sensitive to low-mass sources than the SCUBA data.

\subsection{SED and IRS spectra}

The L 1688 core has been targeted by a large number of continuum surveys, covering wavelengths from 2 to $1300 \mu \mathrm{m}$. 2MASS $(1.25,1.66$ and $2.2 \mu \mathrm{m})$ and Spitzer-IRAC (3.6, 4.5, 5.8 and $8 \mu \mathrm{m})$ and Spitzer-MIPS $(24 \mu \mathrm{m})$ fluxes were obtained from the

\footnotetext{
${ }^{6}$ http://www. submm. caltech.edu/cso/
} 


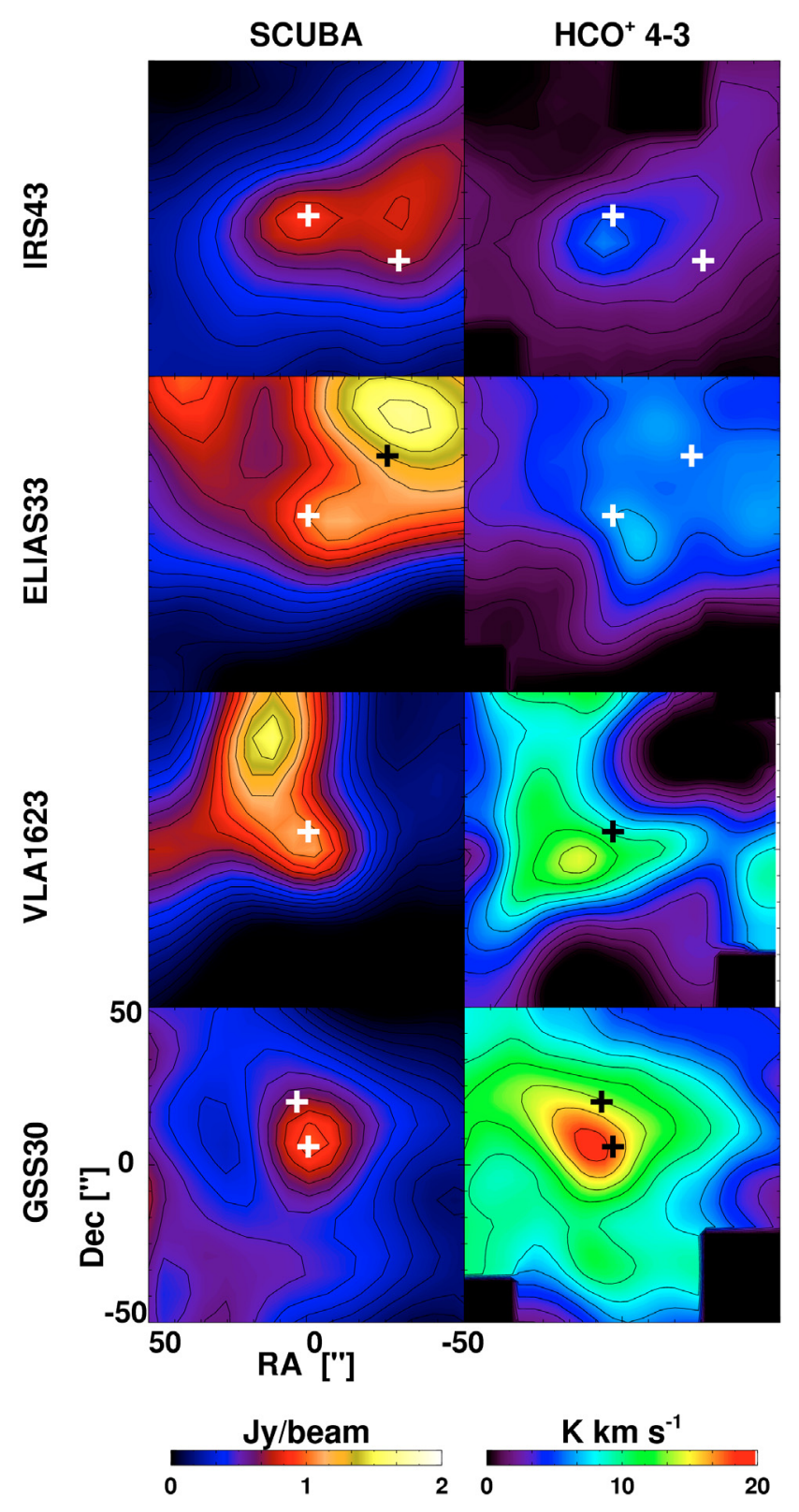

Fig. 2. SCUBA $850 \mu \mathrm{m}$ continuum (left) and $\mathrm{HCO}^{+} 4-3$ (right) integrated intensity maps plotted from bottom to top for GSS 30 IRS1, VLA 1623, Elias 33 and IRS 43. The IR source positions are marked in the SCUBA maps with white crosses. Note that the absolute scale of these maps is a factor 4 higher than those in Figs. 3 and 4. The $850 \mu \mathrm{m}$ continuum flux for VLA 1623 has been scaled down by a factor 4 . In the map of GSS 30, LFAM 1 is located north of the GSS 30 IRS1 position. Elias 32 is located at the north-west side of the Elias 33 map. CRBR 2422.8-3423 is located to the south-west of IRS 43. All four main targets show strong $\mathrm{HCO}^{+}$and SCUBA peaking within $5^{\prime \prime}$ of the IR position at $(0,0)$, characteristic of truly embedded sources.

c2d delivery document (Evans et al. 2007). Recently, Padgett et al. (2008) presented Spitzer-MIPS 70 and $160 \mu$ m fluxes. The MIPS $160 \mu \mathrm{m}$ data were not used in our analysis since a large part of the map was confused by either striping or saturation. For the MIPS $70 \mu \mathrm{m}$ data, we chose to go back to the original data and retrieve the $70 \mu \mathrm{m}$ fluxes source-by-source in a typical $10^{\prime \prime}$ radius using PSF photometry without the extended contribution subtracted. This avoids potential errors in the flux estimates from automatic extraction in crowded or confused regions such as the Ophiuchus core. In addition to the SCUBA
$(850 \mu \mathrm{m})$ and/or SHARC-II (350 $\mu \mathrm{m})$ fluxes, a limited number of sources was also observed using SHARC-II and/or SCUBA at $450 \mu \mathrm{m}$ in a recent survey of disks in the L 1688 core, with fluxes given in the central 15" beam (Andrews \& Williams 2007). Where available, archival data at millimeter wavelengths were used from SEST and IRAM $30 \mathrm{~m}$, as reported in Andrews \& Williams (2007) and originally obtained by André \& Montmerle (1994); Jensen et al. (1996); Nuernberger et al. (1998); Motte et al. (1998) and Stanke et al. (2006).

A number of sources in our sample have also been observed with various observing programs using the IRS instrument on Spitzer at 5-40 $\mu \mathrm{m}(\mathrm{PID}=2,172$ and 179). These spectra are included in this study to confirm the continuum fluxes in the wavelength range of IRS, and to investigate the presence of ice absorption and silicate absorption or emission.

\section{Results}

\subsection{Gas maps}

Figures 2 to 5 present the 21 observed HARP-B fields for $\mathrm{HCO}^{+} 4-3$ and $\mathrm{C}^{18} \mathrm{O} 3-2$, together with the SCUBA $850 \mu \mathrm{m}$ continuum map. Note that the sources in Fig. 2 are much brighter and have different intensity scales. The integrated intensity and peak temperatures for all sources are listed in Table 2, Cols. 2 to 5 . Column 7 states in which figure(s) the sources are mapped or in which figure(s) its spectra are plotted. Figures $6\left(\mathrm{HCO}^{+}\right)$ and $7\left(\mathrm{C}^{18} \mathrm{O}\right)$ show spectra extracted from the maps at the positions of the sources. About $70 \%$ of the sample was observed in $\mathrm{C}^{18} \mathrm{O} 3-2$. The sources for which APEX-2a $\mathrm{HCO}^{+}$spectra were taken can be found in Fig. 8 .

Using the molecular line emission maps, the sample can be divided roughly into three groups. In the first (see Figs. 2 and 3), $\mathrm{HCO}^{+}$peaks at or close to the source positions, with peak intenstities up to a few K. For these sources, GSS 30 IRS1, LFAM 1, VLA 1623, WL 12, LFAM 26, IRS 37, WL 3, WL 17, Elias 33, Elias 32, IRS 44, IRAS 16285 and IRS 54, $\mathrm{HCO}^{+}$seems to be extended on scales of a few $10^{\prime \prime} . \mathrm{C}^{18} \mathrm{O}$ is always present in these maps, and also extended on scales of a few $10^{\prime \prime}$ to $1^{\prime}$.

Note that the $\mathrm{HCO}^{+}$maps in some case show minor offsets w.r.t. the IR positions. For offsets $<8^{\prime \prime}$ this can be attributed to either the pointing accuracy of the JCMT or the differences between IR positions from Bontemps et al. (2001) and c2d. For a few sources, in particular VLA 1623, IRS 44, larger offsets up to $12^{\prime \prime}$ were found. These can be accounted for by potentially three effects. First, self-absorption of $\mathrm{HCO}^{+}$. Second, these sources show strong outflow emission, which peaks off-source and significantly influences the spectrally integrated maps. Third, molecular emission can be suppressed by the freeze-out of molecules in the cold part of the cloud.

A second group (Figs. 4 and 5) lacks detections in $\mathrm{HCO}^{+}$, down to a limit $0.1 \mathrm{~K}$. The sources, C2D-162527.6, WL 19, IRS 46, IRS 48, GY 312 and C2D-162741.6 belong to this group. The third group (Figs. 4 and 5) of sources contains CRBR 2315.8-1700, CRBR 2339.1-2032, WL 2, OphE MM3, IRS 51 and IRS 42. These show $\mathrm{HCO}^{+}$detections at the source position. The $\mathrm{HCO}^{+}$is extended, but there is no sign of a peak at the source positions. For IRS 63 and WL 6, detections of $\mathrm{HCO}^{+}$ are marginal at $\sim 3 \sigma$.

The $\mathrm{HCO}^{+} 4-3$ integrated intensities $\int T_{\mathrm{MB}} \mathrm{d} V$ in protostellar envelopes range from as high as $18.4 \mathrm{~K} \mathrm{~km} \mathrm{~s}^{-1}$ for LFAM 1 to $0.75 \mathrm{~K} \mathrm{~km} \mathrm{~s}^{-1}$ for IRS 63. In Sect. 5.3 the $\mathrm{HCO}^{+} 4-3$ integrated intensities are compared with the column densities derived from the $\mathrm{C}^{18} \mathrm{O} 3-2$. This shows that most embedded sources with 

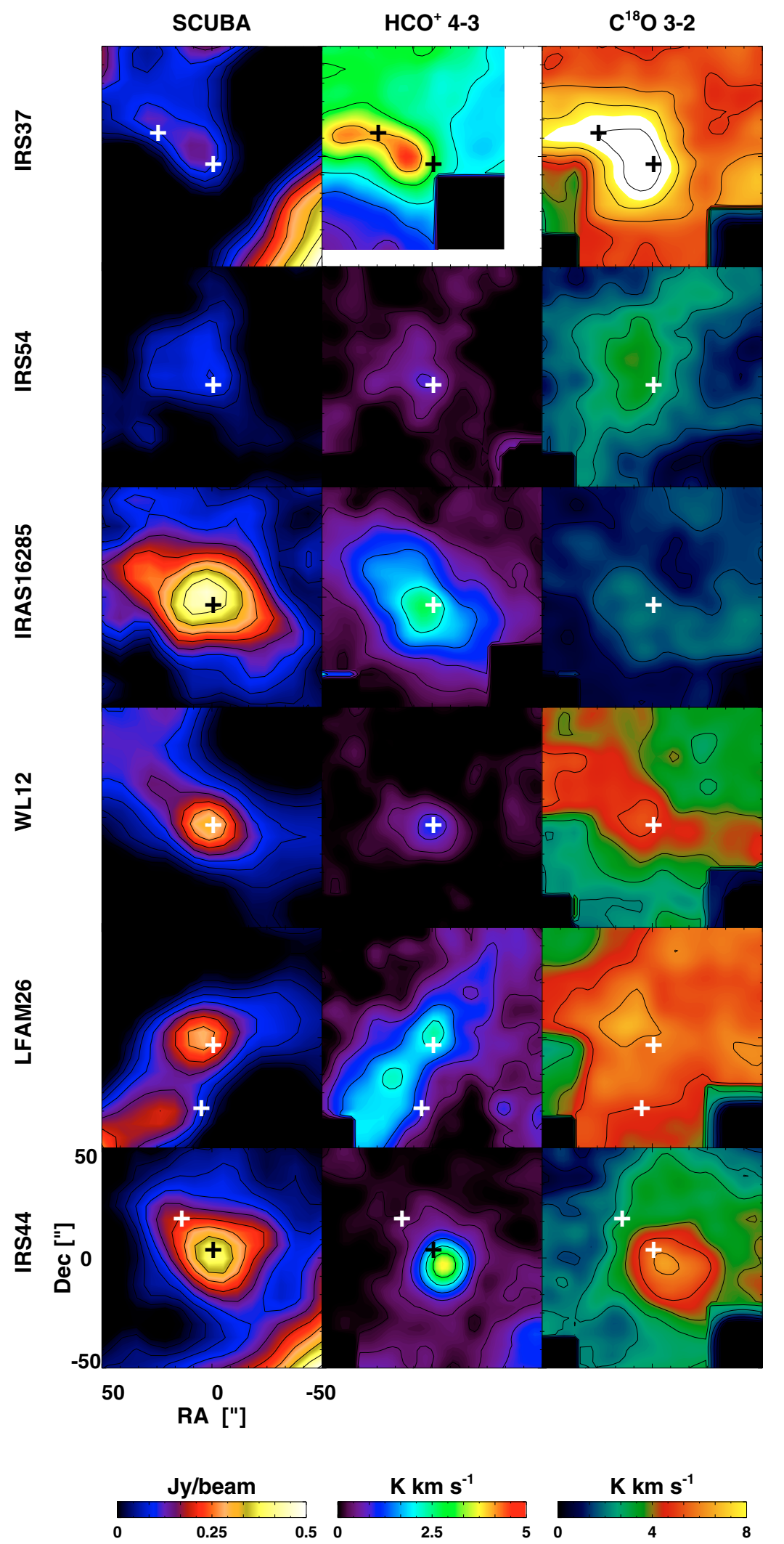

Fig. 3. SCUBA $850 \mu \mathrm{m}$ continuum (left), $\mathrm{HCO}^{+} 4-3$ (middle) and $\mathrm{C}^{18} \mathrm{O}$ 3-2 (right) integrated intensity maps plotted for (from bottom to top) IRS 44, LFAM 26, WL 12, IRAS 16285, IRS 54 and IRS 37. The locations of the IR sources are indicated in the SCUBA maps with white crosses. In the IRS 44 map, IRS 44 is indicated with a black cross, and IRS 46 with a white one. In the LFAM 26 field, OphE MM3 is located south of LFAM 26. In the IRS 37 field, WL 3 is located west of IRS 37. The sources at $(0,0)$ in this figure show weaker $\mathrm{HCO}^{+}$images than in Fig. 2, but are still peaking on the IR positions in both SCUBA and $\mathrm{HCO}^{+}$, and are thus embedded YSOs according to our classification. 

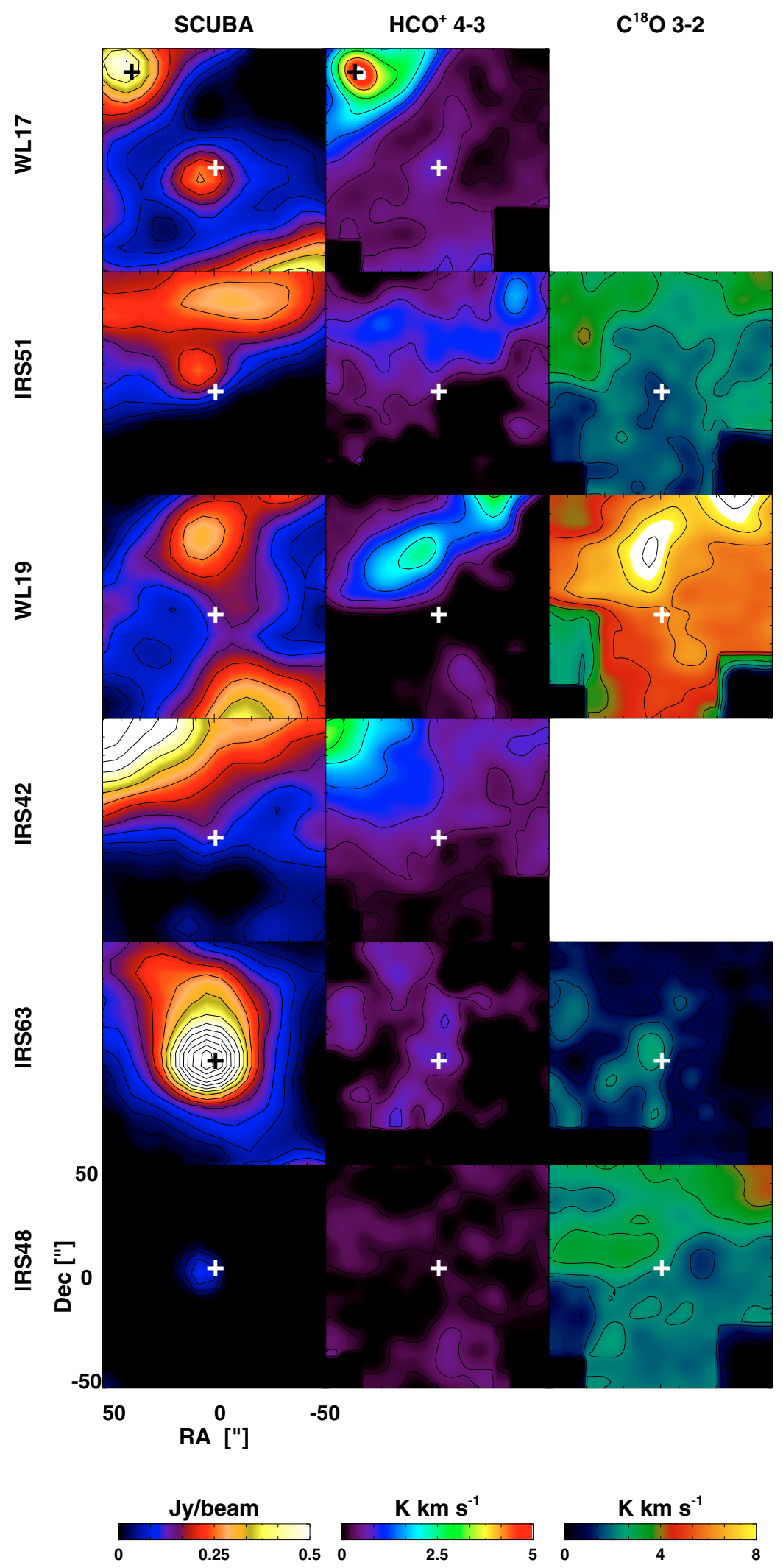

Fig. 4. SCUBA $850 \mu \mathrm{m}$ continuum (left), $\mathrm{HCO}^{+} 4-3$ (middle) and $\mathrm{C}^{18} \mathrm{O} 3-2$ (right) plotted for (from bottom to top) IRS 48, IRS 63, IRS 42, WL 19, IRS 51 and WL17. The locations of the IR sources are indicated in the SCUBA maps with white crosses. The embedded source Elias 29 is shown with a black cross in the field of WL 17. The peak $850 \mu \mathrm{m}$ flux density for IRS 63 is $1.1 \mathrm{Jy} \mathrm{beam}^{-1}$; it was not scaled to accentuate the extended dust emission. The sources at $(0,0)$ in this image are mostly unresolved in SCUBA and show little or no $\mathrm{HCO}^{+}$emission related to the source. They are either transitional or confused sources or (edge-on) disks in our new classidfication, with the exception of IRS 63, which is classified as embedded (transitional). 

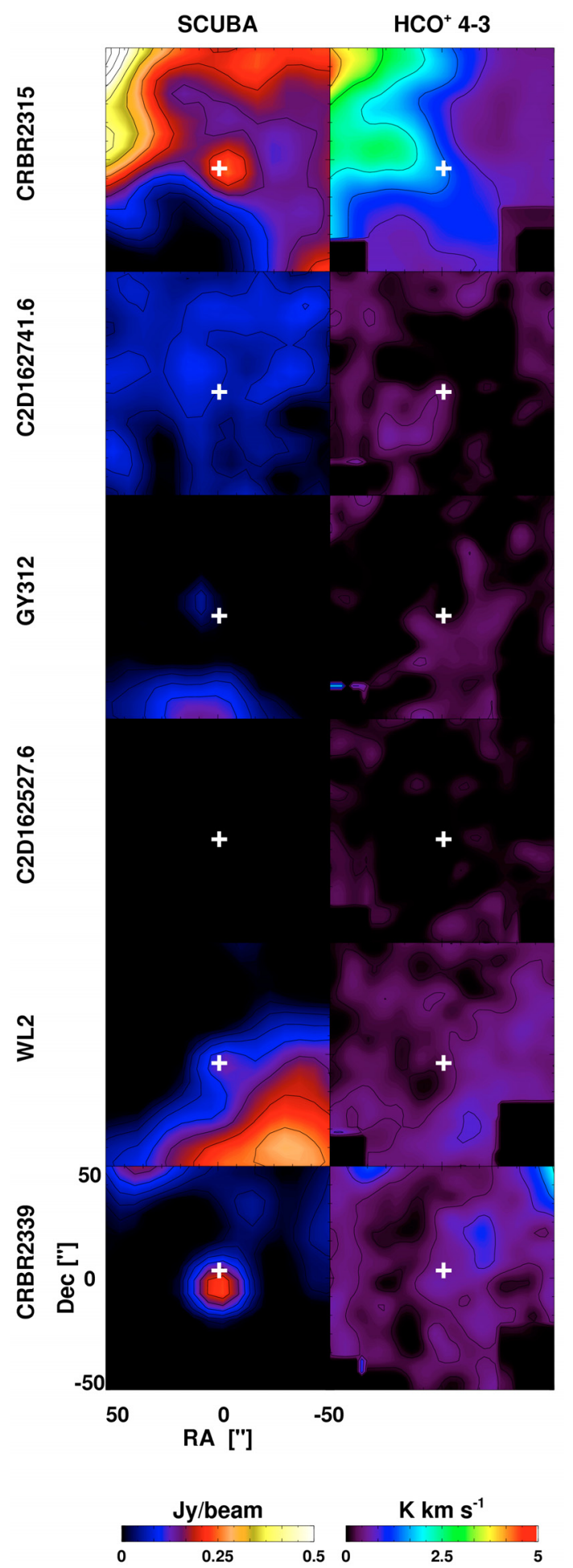

Fig. 5. SCUBA $850 \mu \mathrm{m}$ continuum (left) and $\mathrm{HCO}^{+} 4-3$ (right) plotted for (from bottom to top) CRBR 2339.1, WL 2, C2D 162527.6, GY 312, C2D 162741.6 and CRBR 2315.8. The locations of the sources are indicated in the SCUBA maps with white crosses. These sources have either unresolved or little or no emission in the SCUBA $850 \mu \mathrm{m}$ and $\mathrm{HCO}^{+} 4-3$ maps at $(0,0)$, making them disk sources in our classification.

strong $\mathrm{HCO}^{+}$emission are located in regions with high column densities. The sole exception is IRAS 16285-2355, which is not located in a region with high column density (see Figs. 1 and 3). However, it is possible to find sources with little or no

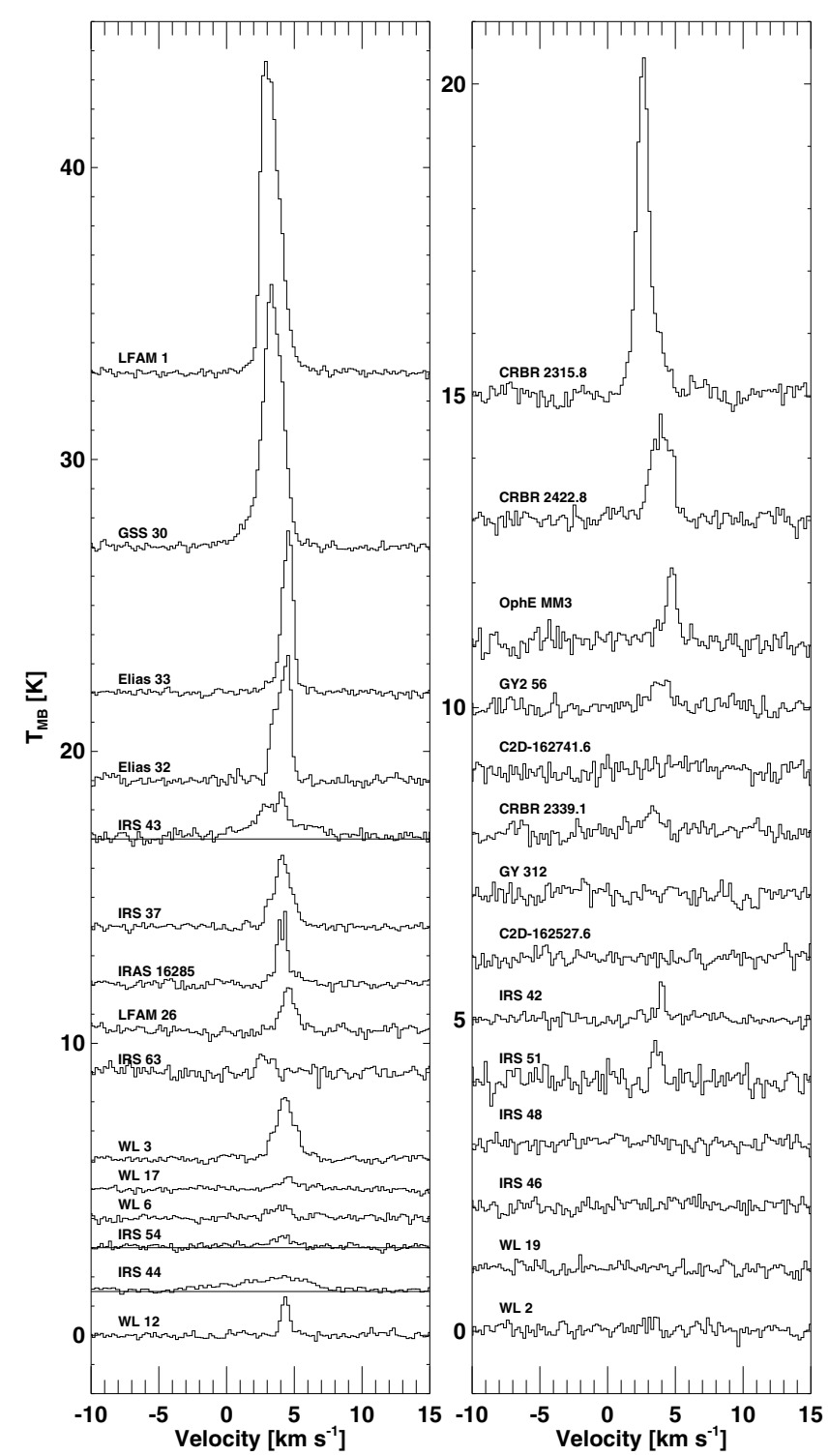

Fig. 6. $\mathrm{HCO}^{+} 4-3$ spectra extracted from the HARP-B maps in a $15^{\prime \prime}$ around the IR positions. Sources classified as embedded in Sect. 6.2 are shown in the left column. In the right column, the sources classified as confused or disks are plotted.

$\mathrm{HCO}^{+}$4-3 emission, such as WL 19, in regions with similarly high column density.

\subsection{Dust maps}

For sources for which molecular emission was taken with the HARP-B array, the spatial extent of the dust is shown in the left column in Figs. 2-5. The sources for which no HARP-B maps were obtained can be found in Fig. 9. The SHARC-II maps are shown in Fig. 10. In these maps, the white contours show the 850 SCUBA emission. The integrated fluxes in a $25^{\prime \prime}$ beam are given in Tables 3 and 4 .

Both the SHARC-II and SCUBA images clearly show that the dust is extended on scales of at least a few arcminutes at many positions in Ophiuchus. The smaller beam and higher frequency of the SHARC-II observations is able to both resolve smaller envelopes (e.g., Elias 33) or confirm that other sources (e.g., WL 20S) are not resolved at $350 \mu \mathrm{m}$ down to $9^{\prime \prime}$. The 
Table 2. Results from HARP-B $\left(\mathrm{HCO}^{+} 4-3\right.$ and $\left.\mathrm{C}^{18} \mathrm{O} 3-2\right)$, APEX-2a $\left(\mathrm{HCO}^{+} 4-3\right)$ and $\mathrm{RxB}\left(\mathrm{C}^{18} \mathrm{O} 3-2\right)$ observations at the source position.

\begin{tabular}{|c|c|c|c|c|c|}
\hline Source & $\begin{array}{c}\mathrm{HCO}^{+} 4-3^{a} \\
\int T_{\mathrm{MB}} \mathrm{d} V \\
{\left[\mathrm{~K} \mathrm{~km} \mathrm{~s}^{-1}\right]}\end{array}$ & $\begin{array}{l}T_{\mathrm{MB}} \\
{[\mathrm{K}]}\end{array}$ & $\begin{array}{c}\mathrm{C}^{18} \mathrm{O} 3-2 \\
\int T_{\mathrm{MB}} \mathrm{d} V \\
{\left[\mathrm{~K} \mathrm{~km} \mathrm{~s}^{-1}\right]}\end{array}$ & $\begin{array}{c}T_{\mathrm{MB}} \\
{[\mathrm{K}]}\end{array}$ & Notes \\
\hline C2D-162527.6 & - & $<0.09$ & - & - & See Fig. 5 \\
\hline GSS 26 & - & - & - & - & See Fig. 9 \\
\hline CRBR 2315.8-1700 & 6.3 & $5.2 *$ & - & - & See Fig. 5 \\
\hline CRBR 2317.3-1925 & $1.5^{b}$ & $2.0^{b}$ & - & - & See Fig. 9 \\
\hline VSSG 1 & - & $<0.4$ & - & - & See Fig. 9 \\
\hline GSS 30 & 16.7 & 9.1 & $10.7^{c}$ & $6.3^{c}$ & See Figs. 2 and 10 \\
\hline LFAM 1 & 18.4 & 10.8 & - & - & See Figs. 2 and 10, GSS 30 maps \\
\hline CRBR 2324.1-1619 & - & - & 14.5 & 12.2 & See Figs. 2 and 10, VLA1623 maps \\
\hline VLA 1623 & 16.2 & 9.0 & 10.4 & $17.9^{d}$ & See Figs. 2 and 10 \\
\hline GY51 & - & - & - & - & See Fig. 9 \\
\hline CRBR 2339.1-2032 & 0.55 & $0.45^{*}$ & - & - & See Fig. 5 \\
\hline WL 12 & 0.87 & 1.4 & 4.8 & 3.9 & See Figs. 3 and 10 \\
\hline WL 2 & 0.3 & $0.18^{*}$ & - & - & See Fig. 5 \\
\hline LFAM 26 & 1.95 & 1.8 & 6.4 & 3.3 & See Fig. 3 \\
\hline WL 17 & 0.6 & 0.45 & - & - & See Fig. 4 \\
\hline Elias 29 & $4.5^{b}$ & $2^{b}$ & $10.4^{e}$ & $4.3^{e}$ & See Fig. 4, WL17 map and Figs. 9 and 10 \\
\hline GY 224 & - & $<0.27^{b}$ & - & - & See Fig. 9 \\
\hline WL19 & - & $<0.1$ & 6.3 & 4.5 & See Fig. 4 \\
\hline WL 20S & - & $<0.26^{b}$ & $5.0^{c}$ & $2.3^{c}$ & See Fig. 10 \\
\hline IRS 37 & 3.7 & 2.5 & 9.55 & 5.7 & See Fig. 3 \\
\hline WL 3 & 3.7 & 2.2 & 8.8 & 6.3 & See Fig. 3, IRS 37 map \\
\hline IRS 42 & 0.45 & $0.7 *$ & 4.0 & $1.2 *$ & See Fig. 4 \\
\hline WL 6 & 0.92 & 0.6 & 5.25 & $4.5^{*}$ & See Figs. 9 and 10 \\
\hline GY 256 & 0.75 & $0.45^{*}$ & 5.4 & $4.5^{*}$ & See Figs. 9 and 10, WL6 maps \\
\hline IRS 43 & $4.5^{d}$ & $1.6^{d}$ & $6.5^{c}$ & $3.2^{c}$ & See Figs. 2, 9 and 10 \\
\hline IRS 44 & $3.4^{d}$ & $0.5^{d}$ & 5.8 & 2.8 & See Fig. 3 \\
\hline Elias 32 & 5.6 & 4.3 & $3.1^{c}$ & $4.5^{c}$ & See Figs. 2 and 10, Elias 33 maps \\
\hline Elias 33 & 6.3 & 5.7 & $6.0^{c}$ & $3.0^{c}$ & See Figs. 2 and 10 \\
\hline IRS 48 & - & $<0.09$ & 2.6 & 2.3 & See Fig. 4 \\
\hline GY 312 & - & $<0.1$ & - & - & See Fig. 5 \\
\hline IRS 51 & 0.75 & 0.75 & 2.0 & $2.8^{*}$ & See Figs. 4 and 10 \\
\hline C2D-162741.6 & - & $<0.1$ & - & - & See Fig. 5 \\
\hline C2D-162748.1 & - & $<0.34^{b}$ & - & - & See Fig. 9 \\
\hline IRS 54 & 0.53 & 0.45 & 3.4 & 3.3 & See Fig. 3 \\
\hline IRAS 16285-2355 & 2.1 & 3.0 & 2.3 & 3.0 & See Fig. 3 \\
\hline C2D-162857.9 & - & $<0.34^{b}$ & - & - & See Fig. 9 \\
\hline IRS 63 & 0.75 & 1.2 & 1.7 & 3.3 & See Fig. 4 \\
\hline \multicolumn{6}{|c|}{ Disks } \\
\hline Haro 1-4 & - & $<0.29^{b}$ & - & - & See Fig. 9 \\
\hline DoAR 25 & - & $<0.28^{b}$ & - & - & See Fig. 9 \\
\hline OphE MM3 & 1.7 & $1.6^{*}$ & 5.5 & $3.0^{*}$ & See Fig. 3, LFAM 26 map \\
\hline SR 21 & - & $<0.28^{b}$ & - & - & See Fig. 9 \\
\hline CRBR 2422.8-3423 & 1.9 & $1.4^{*}$ & - & - & See Fig. 2, IRS 43 map, and Fig. 4, NW corner of IRS 42 \\
\hline IRS 46 & - & $<0.09$ & 2.9 & 2.1 & See Fig. 3, IRS 44 map \\
\hline SR 9 & - & $<0.24^{b}$ & - & - & See Fig. 9 \\
\hline 2Mass 16282 & - & $<0.35^{b}$ & - & - & See Figs. 9 and 10 \\
\hline
\end{tabular}

${ }^{a}$ Intensities marked with a $*$ do not peak at source position.

${ }^{b}$ APEX-2a receiver. Upper limits $(2 \sigma)$ in $0.4 \mathrm{~km} \mathrm{~s}^{-1}$ bin.

${ }^{c}$ JCMT RxB data.

${ }^{d}$ Outflowing gas detected (width $\sim 20 \mathrm{~km} \mathrm{~s}^{-1}$ ).

${ }^{e} \mathrm{C}^{18} \mathrm{O}$ data taken from Boogert et al. (2002), obtained with the CSO.

extended emission originates from cold dust in the parental cloud, as mapped by Motte et al. (1998); Johnstone et al. (2000) and Stanke et al. (2006). This cloud material can exist close to or in the line of sight of many of the Class I sources, but is not necessarily associated with a protostellar envelope, as is often assumed. A good example is the source WL 19 (Fig. 4), where dust emission is found close to the position of the source, but does not peak at the position of WL 19. This emission comes from a prestellar core within the Ophiuchus ridge and not a protostellar envelope.

Based on the spatial extent of the dust, the sample can be divided into four groups. First there are sources with spatially extended dust emission profiles, peaking at the source position. Examples are GSS 30 IRS1, Elias 29 and IRS 43. A second 

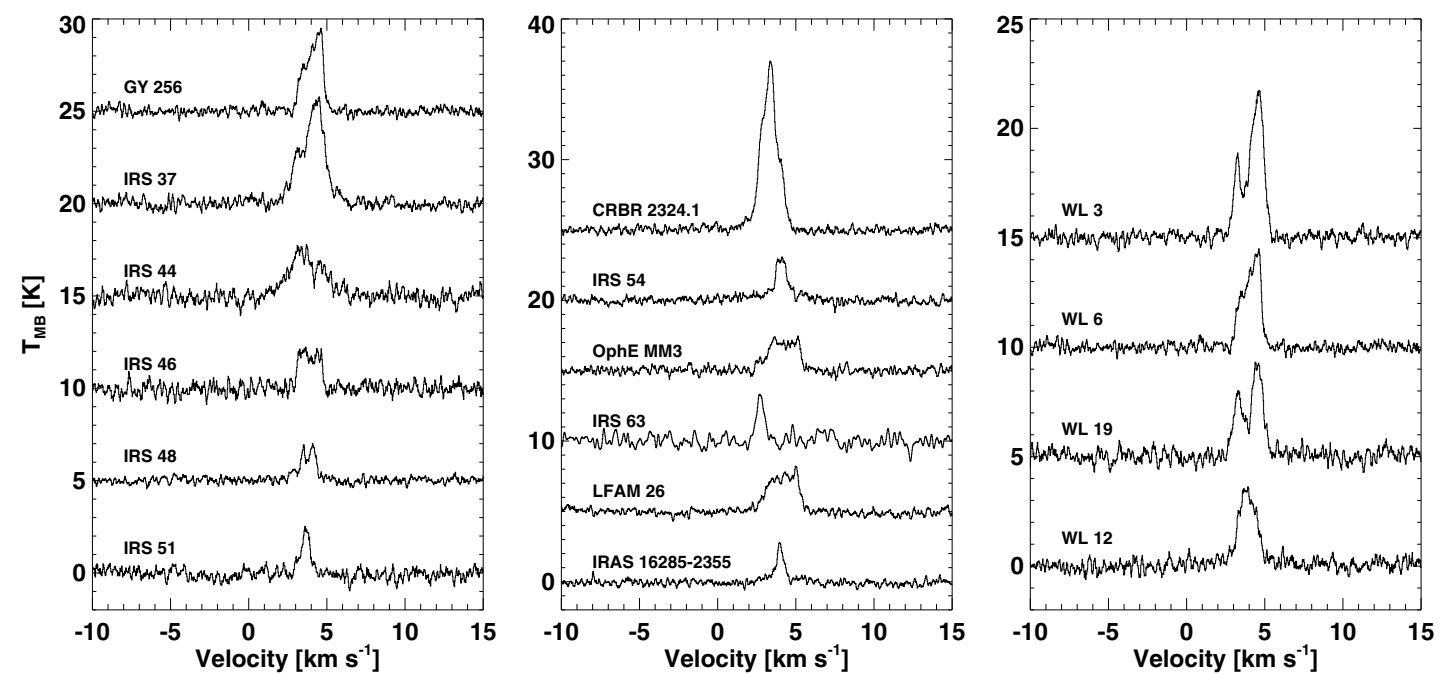

Fig. 7. $\mathrm{C}^{18} \mathrm{O} 3-2$ spectra extracted from the HARP-B maps at the source positions. Spectra are arranged in random order to illustrate the independence of $\mathrm{C}^{18} \mathrm{O}$ to the environment instead of the protostellar envelope.
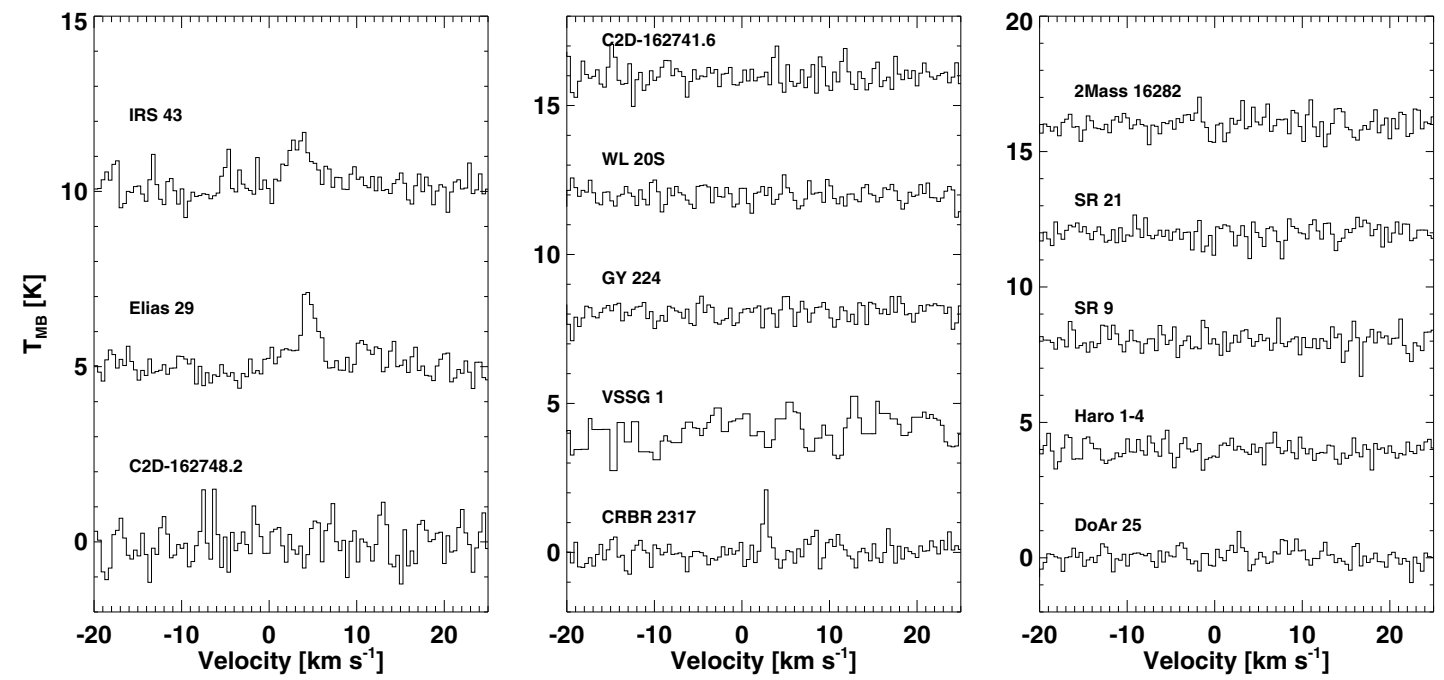

Fig. 8. $\mathrm{HCO}^{+} 4-3$ spectra at the source positions obtained at APEX. Elias 29 and IRS 43, with clear detections, are the only sources found to be embedded in Sect. 6.2. The detection seen for CRBR 2317 is attributed to the cloud material and is classified as a disk.

group shows extended dust emission, but with no central peak at the position of the source. A good example is GY 51 (see Fig. 9), located close to the Oph-A core. Some Class I sources only show unresolved dust emission at the source position, such as IRS 51 (see Fig. 10). A final group shows no emission at 350 or $850 \mu \mathrm{m}$ down to our sensitivity limits. Good examples is Haro 1-4.

Cloud material can contribute significantly or even dominate the emission on the scales probed by SCUBA and SHARC-II. Two fluxes at $850 \mu \mathrm{m}$ are therefore given in Table 4. The first flux is within the $15^{\prime \prime}$ beam obtained from Andrews \& Williams (2007). If no value was given by them, it was extracted from the COMPLETE map for the central beam. Our extracted fluxes agree to within $15 \%$ for sources also listed in Andrews \& Williams (2007), even though we include estimates to reconstruct the chopped-out extended emission. The second flux is extracted from the COMPLETE map for larger apertures encompassing the envelopes, up to a $25^{\prime \prime}$ ( $\left.\sim 3000 \mathrm{AU}\right)$ radius, the typical envelope extent where the temperature and density drop to that of the surrounding cloud. The first number should thus be considered a lower limit on the total envelope and disk emission, while the second is an equivalent upper limit. For disk sources, all emission originating from the source is located within $15^{\prime \prime}$ (<1800 AU).

\subsection{SED and IRS spectra}

Table 4 summarizes all the Spitzer and (sub-)millimeter fluxes. The $2 \mu \mathrm{m}$ to $1.3 \mathrm{~mm}$ flux densities for all 45 sources are plotted in Fig. 11. In addition, low-resolution IRS spectra are overplotted between $\sim 5-30 \mu \mathrm{m}$, where available. In Fig. 12, blowups of the IRS spectra are presented.

\section{Analysis}

\subsection{Concentration}

One method to distinguish embedded YSOs from edge-on disks, starless cores or background sources such as AGB stars and galaxies, is to use a measure of how centrally concentrated the emission is. This method has been used previously in the analysis of SCUBA data (Johnstone et al. 2001; 

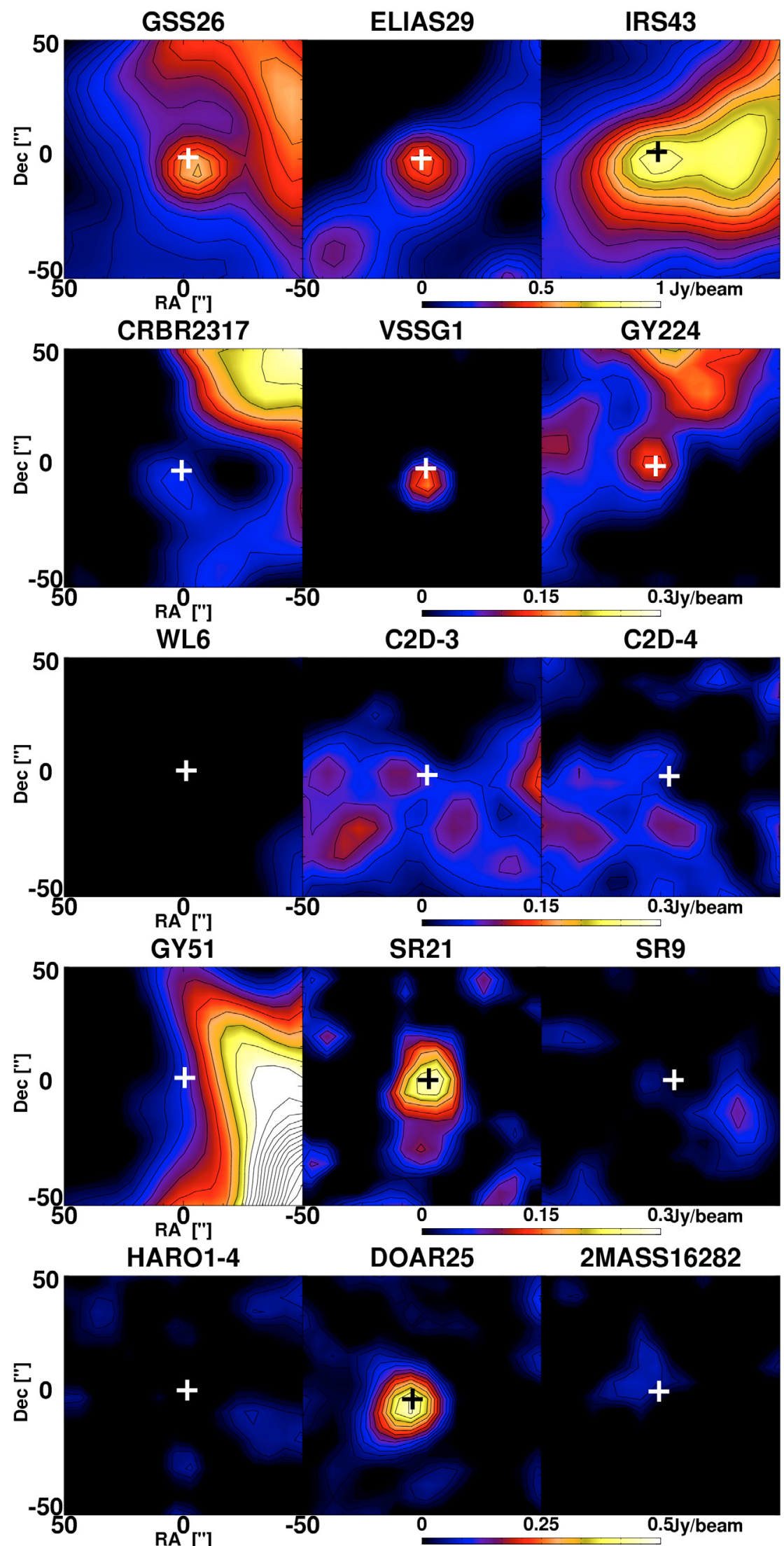

Fig. 9. Dust maps at $850 \mu \mathrm{m}$ extracted from the JCMT-SCUBA COMPLETE map of GSS 26, Elias 29 and IRS 43; CRBR 2317.3-1925, VSSG 1 and GY 224; WL 6, C2D-162748.2 (C2D-3) and C2D-162857.9 (C2D-4); GY 51, SR 21 and SR 9; Haro 1-4, DoAr 25 and 2MASS 16282. Note that the emission toward GY 51 is scaled down by a factor of 4 . 


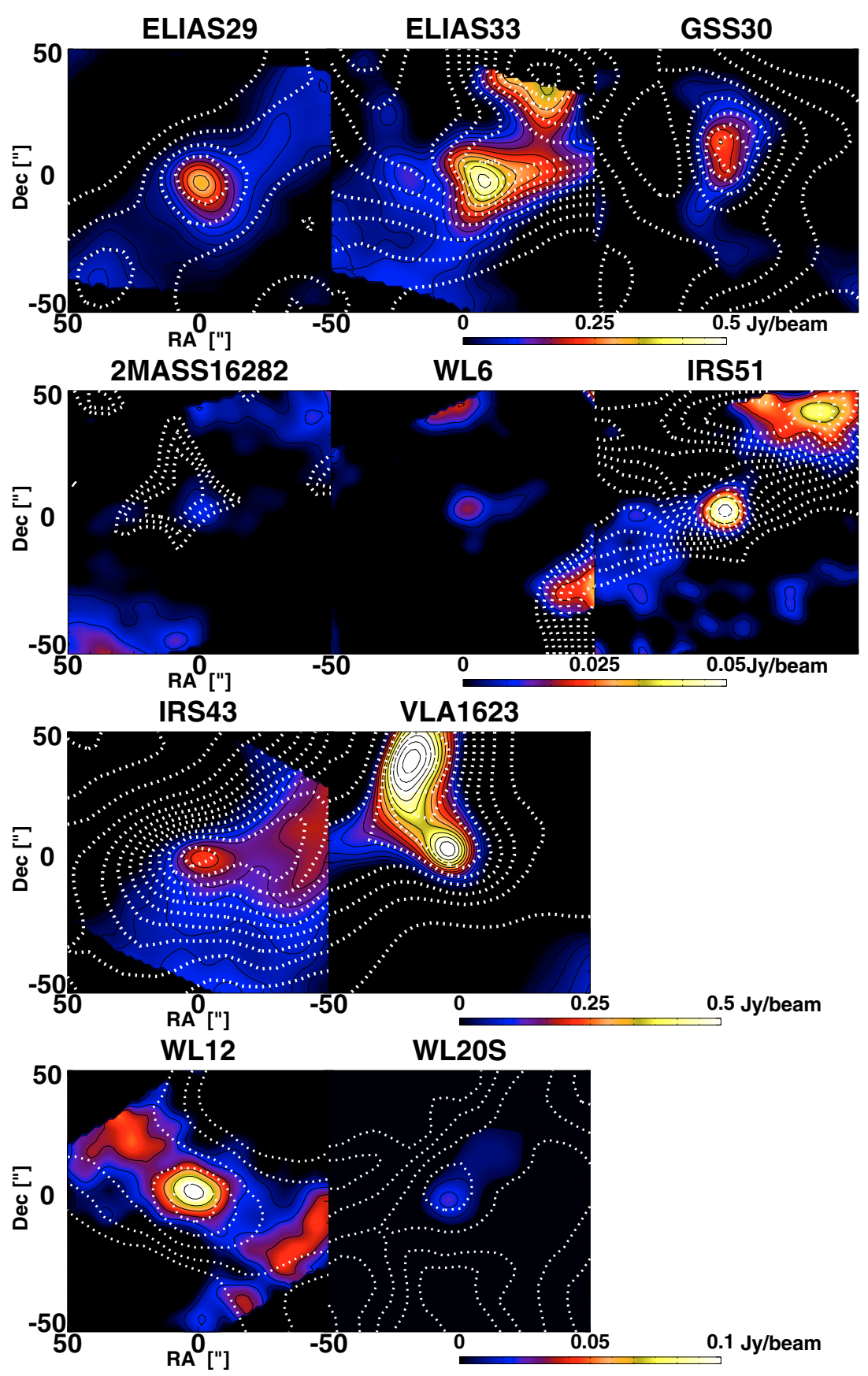

Fig. 10. Dust maps at $350 \mu \mathrm{m}$ observed with the SHARC-II instrument at the CSO. In white, the contours for the JCMT-SCUBA $850 \mu \mathrm{m}$ data are over-plotted, normalized for the maximum emission in the map in contours of $10 \%, 30 \%, 50 \%$ and $70 \%$. The shape of the contour levels at $850 \mu \mathrm{m}$ agrees well with those found at $350 \mu \mathrm{m}$.

Walawender et al. 2005; Jørgensen et al. 2007) by defining a concentration parameter:

$C=1-\frac{1.13 B^{2} S_{850}}{\pi R_{\mathrm{obs}}^{2} F_{0}}$

where $B$ is the beam-size, $S_{850}$ is the total flux at $850 \mu \mathrm{m}$ within the envelope, $R_{\text {obs }}^{2}$ is the observed radius of the envelope and $F_{0}$ is the peak flux of the envelope. The observed radii were selected by calculating the FWHM to the peak flux of each source. The level of concentration potentially provides an indication of the evolutionary stage. Highly concentrated cores $(C>0.75)$ have a very high probability to contain protostars, while cores with low concentration $(C<0.4)$ are often starless, as indicated by the absence of near-IR emission. The observed radii in the $850 \mu \mathrm{m}$ maps were defined by Kirk et al. (2006) as $R=(A / \pi)^{1 / 2}$, with $\mathrm{A}$ the area of the core within the lowest contour of their clumpfind routine. The SHARC-II maps also allow a concentration at $350 \mu \mathrm{m}$ to be made, as seen in Table 3 . These concentrations are found to be within $10 \%$ to those found for the $850 \mu \mathrm{m}$ data, with the exception of WL12 which is more than a factor 2 higher in the SHARC-II $350 \mu \mathrm{m}$ data.

A similar concentration parameter can also be calculated for the distribution of $\mathrm{HCO}^{+}$, using integrated intensities between $V_{\mathrm{LSR}}-5$ to $+5 \mathrm{~km} \mathrm{~s}^{-1}$ :

$C_{\mathrm{HCO}^{+}}=1-\frac{1.13 B^{2} S_{\mathrm{HCO}^{+}}}{\pi R_{\mathrm{obs}}^{2} T_{\mathrm{MB}}}$, 


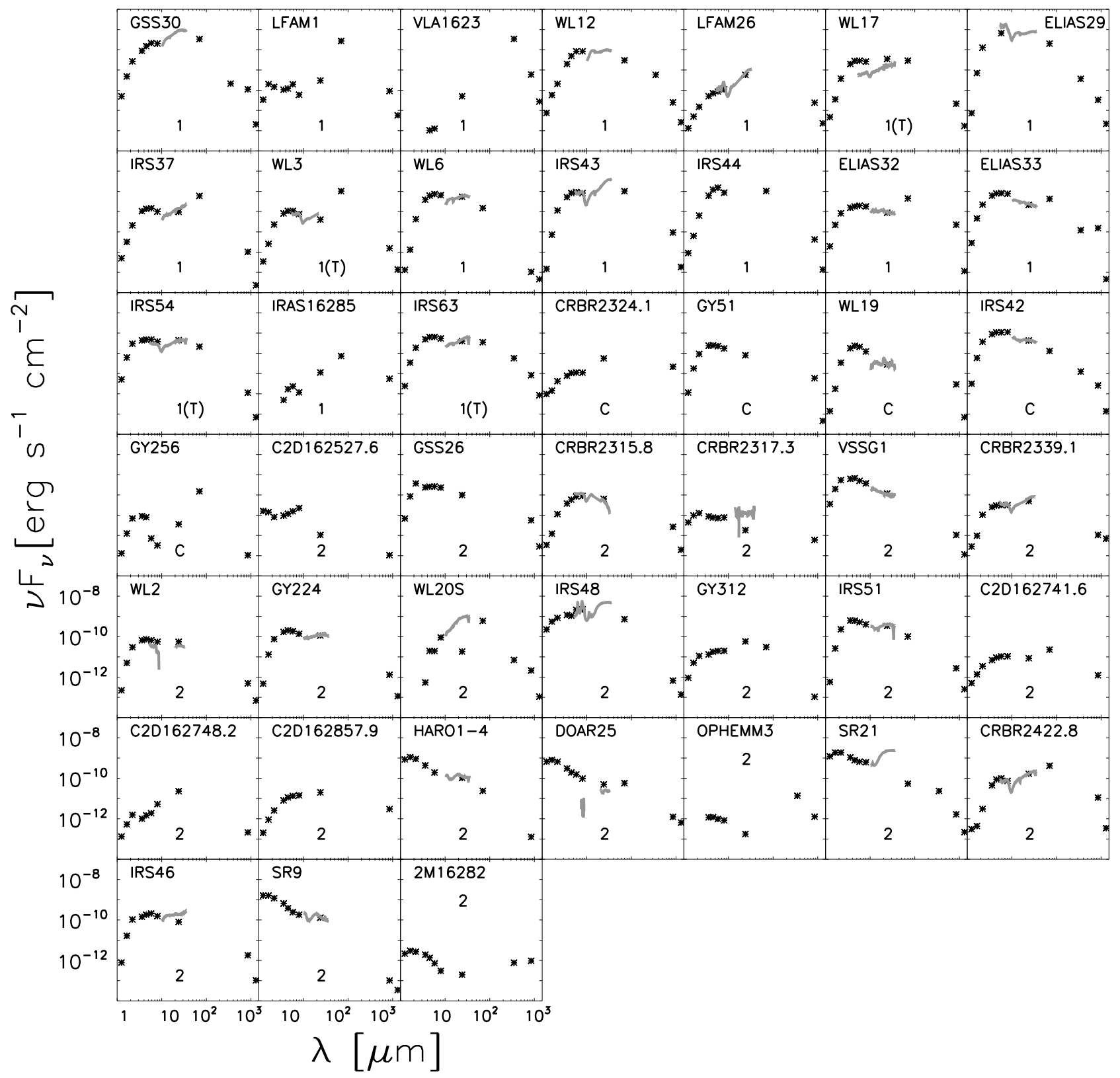

Fig. 11. The spectral energy distributions of all sources, supplemented by spectra taken with IRS. Our classification can be found near the center and bottom of each SED. 1 = Embedded Stage 1, 1(T) = Transitional from embedded to pre-main sequence, $\mathrm{C}=$ confused and $2=($ edge-on $)$ disk.

with $S_{\mathrm{HCO}^{+}}$the spatially and spectrally integrated intensity $\int T_{\mathrm{MB}} \mathrm{d} V$ of $\mathrm{HCO}^{+}$over the entire envelope with radius $R_{\mathrm{obs}}$, and $T_{\mathrm{MB}}$ the peak intensity in the central beam. Since no clumpfind routine was used to characterize the spectral maps, the observed radii of the $\mathrm{HCO}^{+}$concentration were set equal to the FWHM of each spectrally integrated core map. Although different methods to define radii result in different values, tests showed that the corresponding changes in concentration factor are less than $5 \%$.

Tables 3 and 5 list the concentrations of both the $850 \mu \mathrm{m}$ data SCUBA maps and the $\mathrm{HCO}^{+}$HARP-B maps, where available. Although the number of sources with a concentration parameter determined by SCUBA maps is greater than those for $\mathrm{HCO}^{+}$, it is clear that $\mathrm{HCO}^{+}$is more concentrated than the continuum. Figure 13 shows the distribution of the concentration parameters for spatially resolved sources.
Table 3. Source fluxes at $350 \mu \mathrm{m}$ in a $25^{\prime \prime}$ beam observed with SHARC-II at the CSO.

\begin{tabular}{lll}
\hline \hline Source & $\begin{array}{l}\text { Flux } \\
\text { (Jy) }\end{array}$ & Concentration $^{a}$ \\
\hline GSS 30 & 2.5 & 0.58 \\
WL12 & 6.7 & 0.59 \\
Elias 29 & 4.3 & 0.72 \\
VLA1623 & 40 & 0.8 \\
WL20S & 0.82 & 0.64 \\
WL6 & $<0.4$ & $\mathrm{U}$ \\
IRS 43 & 2.1 & 0.62 \\
Elias 33 & 4.4 & 0.55 \\
IRS 51 & 1.4 & 0.64 \\
2MASS 16282 & $<0.2$ & $\mathrm{U}$ \\
\hline
\end{tabular}

${ }^{a}$ See Sect. 5.1; $\mathrm{U}=$ not determined since only flux upper limits obtained. 
Table 4. Source fluxes including IRAC 1-4, MIPS 1-2, SHARC-II, SCUBA and $1.3 \mathrm{~mm}$ data.

\begin{tabular}{|c|c|c|c|c|c|c|c|c|c|c|c|}
\hline Source & $\begin{array}{l}3.6 \mu \mathrm{m}^{e} \\
\text { IRAC I }\end{array}$ & $\begin{array}{l}4.5 \mu \mathrm{m}^{e} \\
\text { IRAC II }\end{array}$ & $\begin{array}{l}5.8 \mu \mathrm{m}^{e} \\
\text { IRACIII }\end{array}$ & $\begin{array}{c}8.0 \mu \mathrm{m}^{e} \\
\text { IRAC IV }\end{array}$ & $\begin{array}{l}24 \mu \mathrm{m}^{e} \\
\text { MIPS I }\end{array}$ & $\begin{array}{c}70 \mu \mathrm{m} \\
\text { MIPS II } \\
\sim 10^{\prime \prime} \\
\text { mJy }\end{array}$ & $\begin{array}{c}350 \mu \mathrm{m} \\
\text { SHARC } \\
9-25^{\prime \prime} b \\
\text { mJy }\end{array}$ & $\begin{array}{c}450 \mu \mathrm{m} \\
\text { SCUBA } \\
10^{\prime \prime b} \\
\mathrm{mJy}\end{array}$ & $\begin{array}{c}850 \mu \mathrm{m} \\
\text { SCUBA } \\
15^{\prime \prime} \\
\mathrm{mJy}\end{array}$ & $\begin{array}{c}850 \mu \mathrm{m} \\
\text { SCUBA } \\
25^{\prime \prime} \\
\mathrm{mJy}\end{array}$ & $\begin{array}{l}15^{\prime \prime} \\
\mathrm{mJy}\end{array}$ \\
\hline C2D-162527.6 & $1.15 \mathrm{e} 1$ & $1.77 \mathrm{e} 1$ & $3.00 \mathrm{e} 1$ & $6.03 \mathrm{e} 1$ & $8.51 \mathrm{e} 1$ & - & - & - & $<3 \mathrm{e} 1$ & $<3 \mathrm{e} 1$ & \\
\hline GSS 26 & $2.86 \mathrm{e} 2 \mathrm{~K}$ & $3.86 \mathrm{e} 2 \mathrm{~K}$ & $5.09 \mathrm{e} 2$ & $6.17 \mathrm{e} 2$ & $7.98 \mathrm{e} 2$ & - & & $6.5 \mathrm{e} 2$ & $3.0 \mathrm{e} 2^{b}$ & $1.6 \mathrm{e} 3$ & 1.e2 \\
\hline CRBR 2315.8-1700 & $4.53 \mathrm{e} 1$ & $8.77 \mathrm{e} 1$ & $1.61 \mathrm{e} 2$ & $2.37 \mathrm{e} 2$ & $5.16 \mathrm{e} 2$ & - & - & & $2.1 \mathrm{e} 2$ & $7.5 \mathrm{e} 2$ & $8.5 \mathrm{e} 1)$ \\
\hline CRBR 2317.3-1925 & $1.05 \mathrm{e} 1$ & $1.15 \mathrm{e} 1$ & $1.39 \mathrm{e} 1$ & $2.05 \mathrm{e} 1$ & $1.46 \mathrm{e} 1$ & - & & & $1.5 \mathrm{e} 2^{b}$ & $1.7 \mathrm{e} 2$ & \\
\hline VSSG 1 & $7.57 \mathrm{e} 2$ & $1.01 \mathrm{e} 3$ & $9.67 \mathrm{e} 2$ & $9.96 \mathrm{e} 2$ & $9.48 \mathrm{e} 2$ & - & & $<6.7 \mathrm{e} 2$ & $2.4 \mathrm{e} 2^{b}$ & $3.0 \mathrm{e} 2$ & $5 \mathrm{e} 1$ \\
\hline GSS 30 & $1.06 \mathrm{e} 3$ & $2.27 \mathrm{e} 3$ & $4.1 \mathrm{e} 3$ & $5.4 \mathrm{e} 3$ & - & $7.83 \mathrm{e} 4$ & $2.5 \mathrm{e} 3^{a}$ & $1.4 \mathrm{e} 3$ & $2.2 \mathrm{e} 2^{b}$ & $3.2 \mathrm{e} 2$ & $9 \mathrm{e} 1$ \\
\hline LFAM 1 & $1.28 \mathrm{e} 1$ & $1.82 \mathrm{e} 1 *$ & $3.78 \mathrm{e} 1$ & $1.59 \mathrm{e} 1 *$ & $2.41 \mathrm{e} 2 *$ & $6.31 \mathrm{e} 4$ & & $2.7 \mathrm{e} 3$ & $5.7 \mathrm{e} 2^{b}$ & $2.6 \mathrm{e} 3$ & $1.1 \mathrm{e} 3$ \\
\hline CRBR 2324.1-1619 & $9.37 \mathrm{e} 0$ & $1.59 \mathrm{e} 1$ & $2.17 \mathrm{e} 1$ & $2.95 \mathrm{e} 1$ & $4.47 \mathrm{e} 2$ & - & & & $8.4 \mathrm{e} 2$ & $6.1 \mathrm{e} 3$ & \\
\hline VLA 1623 & - & $1.57 \mathrm{e}-1 *$ & $2.45 \mathrm{e}-1 *$ & - & $4.04 \mathrm{e} 1$ & - & $4 \mathrm{e} 5^{c}$ & $3.9 \mathrm{e} 5^{c}$ & $5.0 \mathrm{e} 3$ & $1.7 \mathrm{e} 4$ & $1.2 \mathrm{e} 3$ \\
\hline GY51 & $2.86 \mathrm{e} 2$ & $3.71 \mathrm{e} 2$ & $4.38 \mathrm{e} 2$ & $4.66 \mathrm{e} 2$ & $6.39 \mathrm{e} 2 *$ & - & & & $3.3 \mathrm{e} 2$ & $1.7 \mathrm{e} 3$ & $2 \mathrm{e} 1$ \\
\hline CRBR 2339.1-2032 & $3.04 \mathrm{e} 1$ & $4.48 \mathrm{e} 1$ & $6.05 \mathrm{e} 1$ & $8.28 \mathrm{e} 1$ & $3.93 \mathrm{e} 2$ & - & & & $8 \mathrm{e} 1$ & $3 \mathrm{e} 2$ & $3 e 2$ \\
\hline WL 12 & $2.39 \mathrm{e} 2$ & $7.44 \mathrm{e} 2$ & $1.61 \mathrm{e} 3$ & $2.24 \mathrm{e} 3$ & - & $7.0 \mathrm{e} 3$ & $6.7 \mathrm{e} 3^{a}$ & $7.5 \mathrm{e} 2$ & $2.2 \mathrm{e} 2^{b}$ & 7.1e2 & $1.2 \mathrm{e} 2$ \\
\hline WL 2 & $7.92 \mathrm{e} 1$ & $1.11 \mathrm{e} 2$ & $1.28 \mathrm{e} 2$ & $1.48 \mathrm{e} 2$ & $4.46 \mathrm{e} 2$ & - & & & $4 \mathrm{e} 1$ & $1.4 \mathrm{e} 2$ & $3 \mathrm{e} 1$ \\
\hline LFAM 26 & $6.11 \mathrm{e} 0$ & $1.07 \mathrm{e} 1$ & $1.66 \mathrm{e} 1$ & $2.96 \mathrm{e} 1$ & $4.59 \mathrm{e} 2$ & - & & & $2.2 \mathrm{e} 2$ & $6.9 \mathrm{e} 2$ & $1 \mathrm{e} 2$ \\
\hline WL 17 & $2.4 \mathrm{e} 2$ & $4.16 \mathrm{e} 2$ & $5.53 \mathrm{e} 2$ & $6.95 \mathrm{e} 2$ & $2.79 \mathrm{e} 3$ & $6.8 \mathrm{e} 3$ & & $<8.7 \mathrm{e} 2$ & $1 \mathrm{e} 2^{b}$ & $6.1 \mathrm{e} 2$ & $7.5 \mathrm{e} 1$ \\
\hline Elias 29 & - & - & $1.28 \mathrm{e} 4$ & - & - & $<4.7 \mathrm{e} 4$ & $4.3 \mathrm{e} 3^{a}$ & $2.0 \mathrm{e} 3$ & $4.1 \mathrm{e} 2^{b}$ & $9.4 \mathrm{e} 2$ & $9.5 \mathrm{e} 1$ \\
\hline GY 224 & $2.03 \mathrm{e} 2$ & $3.05 \mathrm{e} 2 \mathrm{~K}$ & $3.58 \mathrm{e} 2$ & $3.67 \mathrm{e} 2$ & $9.07 \mathrm{e} 2$ & & & & $1.3 \mathrm{e} 2$ & $3.7 \mathrm{e} 2$ & $5 \mathrm{e} 1$ \\
\hline WL19 & $2.15 \mathrm{e} 2$ & $3.54 \mathrm{e} 2 \mathrm{~K}$ & $4.06 \mathrm{e} 2$ & $3.28 \mathrm{e} 2$ & $2.23 \mathrm{e} 2$ & - & & & $2.0 \mathrm{e} 2$ & $8.3 \mathrm{e} 2$ & $3 \mathrm{e} 1$ \\
\hline WL $20 \mathrm{~S}$ & $6.53 \mathrm{e}-1 * d$ & $2.97 \mathrm{e} 1$ & $3.74 \mathrm{e} 1 *$ & $2.43 \mathrm{e} 2$ & $1.46 \mathrm{e} 2 *$ & $1.4 \mathrm{e} 4$ & $8.2 \mathrm{e} 2^{a}$ & $4.0 \mathrm{e} 2$ & $1.8 \mathrm{e} 2^{b}$ & $6.0 \mathrm{e} 2$ & $4.7 \mathrm{e} 1$ \\
\hline IRS 37 & $1.27 \mathrm{e} 2$ & $2.06 \mathrm{e} 2$ & $2.86 \mathrm{e} 2$ & $2.68 \mathrm{e} 2$ & $7.8 \mathrm{e} 2$ & $1.4 \mathrm{e} 4$ & & $<5.9 \mathrm{e} 2$ & $9.3 \mathrm{e} 1^{b}$ & $3.0 \mathrm{e} 2$ & $<1 \mathrm{e} 1$ \\
\hline WL 3 & $9.86 \mathrm{e} 1$ & $1.63 \mathrm{e} 2$ & $2.08 \mathrm{e} 2$ & $2.04 \mathrm{e} 2$ & $3.25 \mathrm{e} 2$ & $<2.4 \mathrm{e} 4$ & & $<5.8 \mathrm{e} 2$ & $1.2 \mathrm{e} 2^{b}$ & $4.4 \mathrm{e} 2$ & $<6 \mathrm{e} 1$ \\
\hline IRS 42 & $1.06 \mathrm{e} 3$ & $1.63 \mathrm{e} 3$ & $2.1 \mathrm{e} 3$ & $2.98 \mathrm{e} 3$ & $3.45 \mathrm{e} 3$ & $3.0 \mathrm{e} 3$ & $1.5 \mathrm{e} 3^{a}$ & & $1.7 \mathrm{e} 2$ & $7.3 \mathrm{e} 2$ & $<6 \mathrm{e} 1$ \\
\hline WL 6 & $4.67 \mathrm{e} 2 \mathrm{~K}$ & $9.25 \mathrm{e} 2$ & $1.44 \mathrm{e} 3$ & $1.73 \mathrm{e} 3$ & $4.36 \mathrm{e} 3$ & $3.5 \mathrm{e} 3$ & & $1.4 \mathrm{e} 2$ & $<3 \mathrm{e} 1$ & $<3 \mathrm{e} 1$ & $<2 \mathrm{e} 1$ \\
\hline GY 256 & $1.09 \mathrm{e} 1$ & $1.19 \mathrm{e} 1$ & $1.38 \mathrm{e} 0 *$ & $8.58 \mathrm{e}-1^{*}$ & $2.86 \mathrm{e} 1 *$ & $3.5 \mathrm{e} 3$ & & & $<3 \mathrm{e} 1$ & $<3 \mathrm{e} 1$ & \\
\hline IRS 43 & $6.29 \mathrm{e} 2$ & $1.24 \mathrm{e} 3$ & $1.79 \mathrm{e} 3$ & $2.19 \mathrm{e} 3$ & - & $2.4 \mathrm{e} 4$ & $2.1 \mathrm{e} 3^{b}$ & $1.8 \mathrm{e} 3$ & $2.5 \mathrm{e} 2^{b}$ & $2.7 \mathrm{e} 3$ & $8 \mathrm{e} 1$ \\
\hline IRS 44 & 7.31e2 & $1.83 \mathrm{e} 3$ & $2.94 \mathrm{e} 3$ & $2.32 \mathrm{e} 3$ & - & $2.5 \mathrm{e} 4$ & & & $3.6 \mathrm{e} 2$ & $1.2 \mathrm{e} 3$ & $6 \mathrm{e} 1$ \\
\hline Elias 32 & $1.87 \mathrm{e} 2$ & $2.72 \mathrm{e} 2$ & $3.82 \mathrm{e} 2$ & $4.81 \mathrm{e} 2$ & $7.12 \mathrm{e} 2$ & $1.1 \mathrm{e} 4$ & & & $1.7 \mathrm{e} 3$ & $6.4 \mathrm{e} 3$ & $<5 \mathrm{e} 1$ \\
\hline Elias 33 & $7.4 \mathrm{e} 2$ & $1.19 \mathrm{e} 3$ & $1.58 \mathrm{e} 3$ & $2.04 \mathrm{e} 3$ & $1.72 \mathrm{e} 3$ & $<1.0 \mathrm{e} 4$ & $4.4 \mathrm{e} 3^{a}$ & & $1.2 \mathrm{e} 3$ & $4.4 \mathrm{e} 3$ & $<2 \mathrm{e} 1$ \\
\hline IRS 48 & $1.41 \mathrm{e} 3$ & $1.6 \mathrm{e} 3$ & $4.06 \mathrm{e} 3$ & $6.0 \mathrm{e} 3$ & - & $1.7 \mathrm{e} 4$ & & $<1.6 \mathrm{e} 3$ & $1.8 \mathrm{e} 2^{b}$ & $1.9 \mathrm{e} 2$ & $6 \mathrm{e} 1$ \\
\hline GY 312 & $1.58 \mathrm{e} 1$ & $2.67 \mathrm{e} 1$ & $3.82 \mathrm{e} 1$ & $5.32 \mathrm{e} 1$ & $4.61 \mathrm{e} 2$ & 7.1e 2 & & & $<3 \mathrm{e} 1$ & $<3 \mathrm{e} 1$ & \\
\hline IRS 51 & $7.52 \mathrm{e} 2$ & $9.16 \mathrm{e} 2$ & $1.00 \mathrm{e} 3$ & $1.07 \mathrm{e} 3$ & $2.73 \mathrm{e} 3$ & $2.4 \mathrm{e} 3$ & $1.4 \mathrm{e} 3^{a}$ & $<6.8 \mathrm{e} 2$ & $2.2 \mathrm{e} 2$ & $7.8 \mathrm{e} 2$ & $1.1 \mathrm{e} 2$ \\
\hline C2D-162741.6 & $8.31 \mathrm{e} 0$ & $1.39 \mathrm{e} 1$ & $2.04 \mathrm{e} 1$ & $2.86 \mathrm{e} 1$ & $6.89 \mathrm{e} 1$ & $5.3 \mathrm{e} 2$ & & & $1 \mathrm{e} 2$ & $3.5 \mathrm{e} 2$ & \\
\hline 62748.2 & $1.21 \mathrm{e} 0$ & $2.21 \mathrm{e} 0$ & $3.64 \mathrm{e} 0$ & $1.41 \mathrm{e} 1$ & $1.84 \mathrm{e} 2$ & & & & $5 \mathrm{e} 1$ & $6 \mathrm{e} 1$ & \\
\hline IRS 54 & $5.25 \mathrm{e} 2 \mathrm{~K}$ & $7.12 \mathrm{e} 2$ & $9.31 \mathrm{e} 2$ & $1.01 \mathrm{e} 3$ & $3.56 \mathrm{e} 3$ & $5.0 \mathrm{e} 3$ & & & $1 \mathrm{e} 2$ & $3.2 \mathrm{e} 2$ & $3 \mathrm{e} 1$ \\
\hline IRAS 16285 & $5.89 \mathrm{e}-1$ & $2.55 \mathrm{e} 0$ & $4.49 \mathrm{e} 0 \mathrm{~K}$ & $3.13 \mathrm{e} 0$ & $9.01 \mathrm{e} 1$ & $1.7 \mathrm{e} 3$ & & & $5 \mathrm{e} 2$ & $1.6 \mathrm{e} 3$ & \\
\hline C2D-162857.9 & $9.73 \mathrm{e} 0$ & $1.7 \mathrm{e} 1$ & $2.57 \mathrm{e} 1$ & $3.83 \mathrm{e} 1$ & $1.61 \mathrm{e} 2$ & - & & & $5 \mathrm{e} 1$ & $8.5 \mathrm{e} 2$ & \\
\hline IRS 63 & $5.89 \mathrm{e} 2$ & $9.57 \mathrm{e} 2$ & $1.25 \mathrm{e} 3$ & $1.45 \mathrm{e} 3$ & $3.23 \mathrm{e} 3$ & $8.3 \mathrm{e} 3$ & $6.7 \mathrm{e} 3^{b}$ & $4.7 \mathrm{e} 3$ & $1.0 \mathrm{e} 3^{b}$ & $2.4 \mathrm{e} 3$ & $3.7 \mathrm{e} 2$ \\
\hline \multicolumn{12}{|c|}{ Disks } \\
\hline Haro 1-4 & $5.19 \mathrm{e} 2$ & - & $3.73 \mathrm{e} 2$ & - & $8.39 \mathrm{e} 2$ & $5.6 \mathrm{e} 2$ & & & - & - & \\
\hline DoAR 25 & $3.67 \mathrm{e} 2$ & $2.92 \mathrm{e} 2$ & $2.99 \mathrm{e} 2$ & $2.58 \mathrm{e} 2$ & $3.99 \mathrm{e} 2$ & $1.4 \mathrm{e} 3$ & & & $4.6 \mathrm{e} 2^{b}$ & $5.2 \mathrm{e} 2$ & $2.8 \mathrm{e} 2$ \\
\hline OphE MM3 & $1.42 \mathrm{e} 0$ & $1.79 \mathrm{e} 0$ & $1.88 \mathrm{e} 0 *$ & $2.22 \mathrm{e} 0$ & $1.41 \mathrm{e} 0 *$ & - & $1.6 \mathrm{e} 3^{a}$ & $5.4 \mathrm{e} 2$ & $1.6 \mathrm{e} 2$ & $3.6 \mathrm{e} 2$ & \\
\hline SR 21 & $1.3 \mathrm{e} 3$ & $1.17 \mathrm{e} 3$ & $1.28 \mathrm{e} 3$ & $1.66 \mathrm{e} 3$ & - & $1.3 \mathrm{e} 3$ & $2.8 \mathrm{e} 3^{b}$ & $1.9 \mathrm{e} 3$ & $4.0 \mathrm{e} 2^{b}$ & $4.7 \mathrm{e} 2$ & $9.5 \mathrm{e} 1$ \\
\hline CRBR 2422.8-3423 & $5.4 \mathrm{e} 1$ & $1.31 \mathrm{e} 2$ & $1.91 \mathrm{e} 2$ & $1.98 \mathrm{e} 2$ & $1.34 \mathrm{e} 3$ & $9.8 \mathrm{e} 3$ & & & $1.3 \mathrm{e} 3$ & $3.2 \mathrm{e} 3$ & $1.5 \mathrm{e} 2$ \\
\hline IRS 46 & $1.72 \mathrm{e} 2 \mathrm{~K}$ & $2.71 \mathrm{e} 2$ & $4.02 \mathrm{e} 2$ & $4.11 \mathrm{e} 2$ & $6.39 \mathrm{e} 2 *$ & - & $1.4 \mathrm{e} 3^{a}$ & & $1.8 \mathrm{e} 2$ & $5.1 \mathrm{e} 2$ & $4.5 \mathrm{e} 1$ \\
\hline SR 9 & $7.84 \mathrm{e} 2$ & $5.75 \mathrm{e} 2$ & $4.65 \mathrm{e} 2$ & $4.84 \mathrm{e} 2$ & $1.04 \mathrm{e} 3$ & - & & & $<25^{b}$ & $2.9 \mathrm{e} 1$ & $1.5 \mathrm{e} 1$ \\
\hline 2Mass 16282 & $2.32 \mathrm{e} 0$ & $2.00 \mathrm{e} 0$ & $1.39 \mathrm{e} 0$ & $8.15 \mathrm{e}-1$ & $1.58 \mathrm{e} 0$ & - & $9 \mathrm{e} 1^{b}$ & $8 \mathrm{e} 1$ & $5.8 \mathrm{e} 1$ & $2.7 \mathrm{e} 2$ & \\
\hline
\end{tabular}

${ }^{a}$ This paper; ${ }^{b} 350 / 450 \mu \mathrm{m}$ flux taken from Andrews \& Williams (2007). Fluxes obtained with SHARC-II are given either in a $9^{\prime \prime}$ beam (Andrews \& Williams 2007) or in a 25" radius (This work); ${ }^{c}$ data taken from André et al. (1993); ${ }^{d}$ two sources within $4^{\prime \prime}$ with a total flux of $2 \mathrm{e} 2 \mathrm{mJy}$; ${ }^{e}$ IRAC and MIPS-24 Fluxes marked with “*” are band-filled sources with a $S / N<5$ or sources with $S / N<5$. Such limits are caused either by saturation or a very low emission. A "K" added to the flux indicates that the obtained flux was flagged as "Complex", with multiple detection within 2-3".

\subsection{Environment}

In contrast with deeply embedded Class 0 YSOs, the environment around the embedded Class I YSOs has a large influence on the analysis of the generally weaker source data. Figure 7 in Boogert et al. (2002) illustrates how complex the environment can become. Elias 29, an embedded YSO, is located in a dense ridge of material, but in front of that ridge, two foreground layers were identified using the emission from a dozen different molecules. Continuum emission and ice absorption originate in all these layers. For most of our sources, however, the situation appears to be less complex. Figures 6 and 7 show that the $\mathrm{HCO}^{+} 4-3$ profiles are mostly single peaked. However, $\mathrm{C}^{18} \mathrm{O} 3-2$ often shows a more complex profile with multiple peaks. 
Table 5. Source properties derived from the gas and dust. The table has been rearranged compared with Table 1 to reflect the classification in Sect. 6.2

\begin{tabular}{|c|c|c|c|c|c|c|c|c|}
\hline Source & $\alpha_{\mathrm{IR}}$ & $\begin{array}{l}N_{\mathrm{H}_{2}}^{a} \\
\left(10^{22} \mathrm{~cm}^{-2}\right)\end{array}$ & $\Delta \alpha^{b}$ & $\begin{array}{l}\text { Mass }^{c} \\
\left(10^{-2} M_{\odot}\right)\end{array}$ & $\begin{array}{l}L_{\mathrm{bol}} \\
\left(10^{-1} L_{\odot}\right)\end{array}$ & $\begin{array}{l}T_{\text {bol }} \\
(\mathrm{K}) \\
\end{array}$ & $C_{850^{d}}$ & $C_{\mathrm{HCO}^{+}}{ }^{d}$ \\
\hline & \multicolumn{8}{|c|}{ Embedded sources } \\
\hline $\operatorname{GSS} 30^{e}$ & 1.46 & $23.3 / 19.4 *$ & 2.6 & 20.5 & 33 & 123 & 0.55 & 0.69 \\
\hline LFAM $1^{e}$ & 0.73 & $50 *$ & 2.6 & 17.1 & 8.3 & 86 & 0.59 & 0.69 \\
\hline VLA 1623 & no & $441 *$ & - & 111 & 2.7 & 12 & 0.7 & 0.72 \\
\hline WL 12 & 2.49 & $10.5 / 15.2 *$ & 3.1 & 4.6 & 34 & 155 & 0.26 & 0.81 \\
\hline LFAM 26 & 1.27 & $14.0 / 18.5^{*}$ & 4.4 & 4.5 & 0.44 & 238 & 0.40 & 0.69 \\
\hline WL 17 & 0.61 & $8.4^{*}$ & 1.0 & 4.0 & 6.7 & 323 & 0.22 & 0.71 \\
\hline Elias 29 & 1.69 & $22.7 / 33.6^{*}$ & 1.5 & 6.2 & 25 & 424 & 0.49 & $-^{f}$ \\
\hline IRS $37^{e}$ & 0.25 & 20.8 & 1.0 & 1.2 & 3.8 & 243 & 0.58 & 0.72 \\
\hline WL $3^{e}$ & -0.03 & $19.2 / 9.7 *$ & 2.3 & 2.9 & 4.6 & 192 & 0.25 & 0.67 \\
\hline WL 6 & 0.72 & 11.4 & - & $<0.4$ & 8.5 & 394 & $\mathrm{U}$ & 0.77 \\
\hline IRS 43 & 1.17 & $14.2 / 20.7 *$ & 2.3 & 17.1 & 10 & 134 & 0.58 & 0.79 \\
\hline IRS 44 & 2.29 & $12.6 / 30.2 D^{*}$ & 2.9 & 8.0 & 11 & 140 & 0.33 & 0.80 \\
\hline Elias 32 & -0.03 & $6.8 / 140 *$ & 3.7 & 41.9 & 5 & 321 & 0.37 & 0.70 \\
\hline Elias 33 & -0.12 & $13.1 / 96^{*}$ & 3.1 & 28.2 & 12 & 460 & 0.6 & 0.64 \\
\hline IRS 54 & 0.03 & 7.4 & 1.2 & 3.1 & 7.8 & 486 & 0.8 & 0.80 \\
\hline IRAS 16285-2355 & 1.23 & $5.0 / 41.9 \mathrm{D}^{*}$ & 3.1 & 10.3 & 0.57 & 77 & 0.45 & 0.80 \\
\hline \multirow[t]{2}{*}{ IRS 63} & 0.14 & $3.7 / 84.0 \mathrm{D}^{*}$ & - & 16.4 & 13 & 363 & 0.75 & 0.60 \\
\hline & \multicolumn{8}{|c|}{ Confused sources } \\
\hline CRBR 2324.1-1619 & 0.87 & $31.6 / 70.5^{*}$ & 5.2 & 39.3 & 0.44 & 283 & $\mathrm{U}$ & $\mathrm{U}$ \\
\hline GY51 & 0.05 & $27.7 *$ & 4.5 & 11.1 & 2.3 & 706 & $\mathrm{U}$ & $\mathrm{U}$ \\
\hline WL19 & -0.43 & $13.7 / 16.8^{*}$ & 4.9 & 5.5 & 1.5 & 730 & 0.15 & 0.53 \\
\hline IRS 42 & -0.03 & $8.7 / 14.2^{*}$ & 1.3 & 4.7 & 14 & 540 & 0.44 & 0.60 \\
\hline \multirow{2}{*}{ GY 256} & -0.05 & 11.8 & - & $<0.2$ & 1.1 & 104 & $\mathrm{U}$ & 0.70 \\
\hline & \multicolumn{2}{|c|}{$\overline{\text { Disks }}$} & & & & & & \\
\hline C2D-162527.6 & 0.36 & - & - & $<0.4$ & 0.16 & 1051 & $\mathrm{U}$ & $\mathrm{U}$ \\
\hline GSS 26 & -0.46 & $24.3 D^{*}$ & 1.8 & 10.2 & 3.2 & 877 & 0.65 & $\mathrm{U}$ \\
\hline CRBR 2315.8-1700 & 0.69 & $17.6^{*}$ & 1.0 & 5.1 & 0.93 & 456 & 0.38 & 0.74 \\
\hline CRBR 2317.3-1925 & -0.56 & $12.6^{*}$ & 0.3 & 1.1 & 0.11 & 1105 & 0.85 & $\mathrm{~S}$ \\
\hline VSSG 1 & -0.73 & $20.1^{*}$ & - & 2.0 & 5.8 & 930 & 0.74 & SU \\
\hline CRBR 2339.1-2032 & 0.45 & $6.7 *$ & - & 2.0 & 0.55 & 426 & 0.55 & 0.58 \\
\hline WL 2 & 0.02 & - & - & 1.0 & 0.88 & 573 & 0.3 & 0.58 \\
\hline GY 224 & -0.05 & $10.9 *$ & 1.0 & 2.4 & 2.1 & 614 & 0.63 & SU \\
\hline WL 20S & 2.75 & $10.9 / 15.3 D^{*}$ & 1.0 & 3.9 & 4.5 & 85 & 0.57 & SU \\
\hline IRS 48 & 0.88 & $5.7 / 15.2 \mathrm{D}^{*}$ & 2.1 & 1.2 & 81 & 238 & 0.70 & $\mathrm{U}$ \\
\hline GY 312 & 0.64 & - & - & $<0.2$ & 0.73 & 285 & $\mathrm{U}$ & 0.3 \\
\hline IRS 51 & -0.15 & 4.4/18.4D* & 2.3 & 5.0 & 8 & 548 & 0.27 & 0.59 \\
\hline C2D-162741.6 & 0.32 & - & - & 2.3 & 0.28 & 279 & $\mathrm{U}$ & $\mathrm{U}$ \\
\hline C2D-162748.2 & 1.55 & - & - & 0.4 & 0.17 & 241 & $\mathrm{U}$ & SU \\
\hline C2D-162857.9 & 0.67 & - & - & 5.6 & 0.22 & 402 & $\mathrm{U}$ & SU \\
\hline Haro 1-4 & -0.89 & - & - & 0.45 & 5.6 & 762 & $\mathrm{U}$ & SU \\
\hline DoAR 25 & -1.12 & $38.6^{*}$ & - & 3.4 & 4.7 & 1422 & 0.90 & SU \\
\hline OphE MM3 & -0.33 & $12.0 / 13.4 *$ & 3.4 & 2.3 & 0.17 & 45 & $\mathrm{U}$ & 0.68 \\
\hline SR 21 & -0.79 & $33.6^{*}$ & - & 3.1 & 1.8 & 1070 & 0.89 & SU \\
\hline CRBR 2422.8-3423 & 1.01 & $106.6^{*}$ & 2.3 & 20.5 & 4.4 & 157 & $\mathrm{U}$ & $-^{f}$ \\
\hline IRS 46 & 0.18 & $6.3 / 15.1^{*}$ & 2.8 & 3.3 & 1.9 & 684 & $\mathrm{U}$ & SU \\
\hline SR 9 & -1.07 & - & - & $<0.4$ & 8.7 & 1588 & $\mathrm{U}$ & SU \\
\hline 2MASS 16282 & -1.55 & - & - & 1.7 & 0.84 & 66 & $\mathrm{U}$ & SU \\
\hline
\end{tabular}

${ }^{a}$ Column densities marked with * are calculated from the SCUBA dust maps. Embedded sources where a large disk contribution is likely are indicated with a D. Note that no column densities could be calculated from the dust below $1 \times 10^{23} \mathrm{~cm}^{-2}$.

${ }^{b} \Delta \alpha$ is an upper limit to change in 2-24 $\mu \mathrm{m}$ spectral slope due to the reddening by foreground clouds at this source. This is derived by considering the mean column density at $45^{\prime \prime}$ off the source position.

${ }^{c}$ Upper limit derived from the $850 \mu \mathrm{m}$ data using the relation from Shirley et al. (2000), with the assumption that all $850 \mu \mathrm{m}$ emission originates within a protostellar envelope.

${ }^{d} \mathrm{U}=$ Undetected or Unobserved, $\mathrm{S}=$ single position spectrum available only.

${ }^{e}$ Circumbinary envelope.

${ }^{f}$ Although both Elias 29 and CRBR 2422.8-3423 were observed, these sources are located near the edge of a field and do not have sufficient coverage to obtain a concentration parameter for the $\mathrm{HCO}^{+}$.

LFAM 26, located at the top of the Ophiuchus ridge only a few arcmin north-east of Elias 29 , is an especially complex case. Figure 14 shows the spectral map in $17^{\prime \prime}$ spatial bins for
$\mathrm{HCO}^{+} 4-3$ and $\mathrm{C}^{18} \mathrm{O} 3-2$ maps. It is clearly seen that besides the integrated emission (Fig. 3), the line profiles of $\mathrm{HCO}^{+}$and $\mathrm{C}^{18} \mathrm{O}$ both vary over the entire map. The $\mathrm{HCO}^{+}$spectra show mostly 

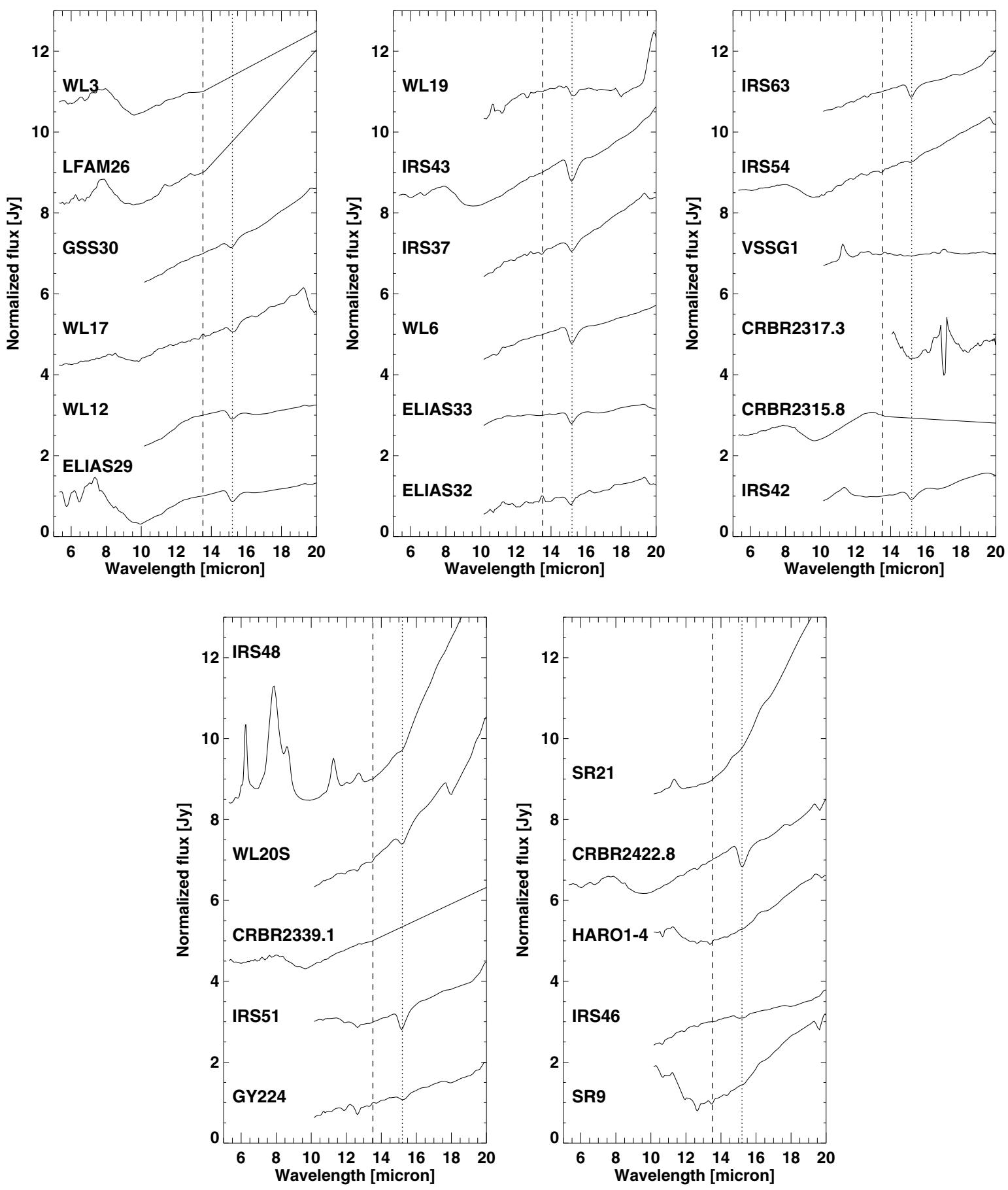

Fig. 12. The individual IRS spectra (where available). Each spectra is plotted from 5 to $20 \mu \mathrm{m}$. All spectra are normalised to their own emission at 13.5 micron, indicated with a vertical dashed line.

single gaussians. At the position of LFAM 26, the $\mathrm{HCO}^{+}$profile has a small outflow signature on the blue side of the spectrum. The $\mathrm{C}^{18} \mathrm{O}$ emission requires at least four distinct components (Fig. 15) at 2.7, 3.5, 4.4 and $5.0 \mathrm{~km} \mathrm{~s}^{-1}$. The three components at $2.7,3.5$ and $5.0 \mathrm{~km} \mathrm{~s}^{-1}$ are the same as found in Boogert et al. (2002) at the position of Elias 29. Most lines observed in Boogert et al. (2002) peak short-wards of $5.0 \mathrm{~km} \mathrm{~s}^{-1}$ component, which could be indicative of the $4.4 \mathrm{~km} \mathrm{~s}^{-1}$ component. However, the difference between these two components is very pronounced in the spectra in Figs. 14 and 15 at the position of LFAM 26 so that we treat them as separate. $\mathrm{HCO}^{+}$is aligned with the $4.4 \mathrm{~km} \mathrm{~s}^{-1}$ component.
Using the molecular excitation program RADEX (van der Tak et al. 2007), one can calculate the expected average contribution from cloud material to the $\mathrm{HCO}^{+} 4-3$ emission. Using a $\mathrm{HCO}^{+}$abundance of $10^{-8}$ with respect to $\mathrm{H}_{2}$, a density of $10^{4} \mathrm{~cm}^{-3}$, a cloud temperature of $15 \mathrm{~K}$, a width of $1 \mathrm{~km} \mathrm{~s}^{-1}$ and a $\mathrm{H}_{2}$ column density of $10^{22} \mathrm{~cm}^{-2}\left(A_{\mathrm{V}} \approx 10\right)$, a contribution of $\sim 0.1 \mathrm{~K} \mathrm{~km} \mathrm{~s}^{-1}$ is found. Only at higher column $\left(\sim 10^{23} \mathrm{~cm}^{-2}\right)$ or volume densities $\left(\sim 1 \times 10^{5} \mathrm{~cm}^{-3}\right)$ are contributions found from the cloud on the order of $1 \mathrm{~K} \mathrm{~km} \mathrm{~s}^{-1}$, comparable to the observed $4.4 \mathrm{~km} \mathrm{~s}^{-1}$ emission at off-positions such as $(-50,50)$. This is in agreement with the numbers given 


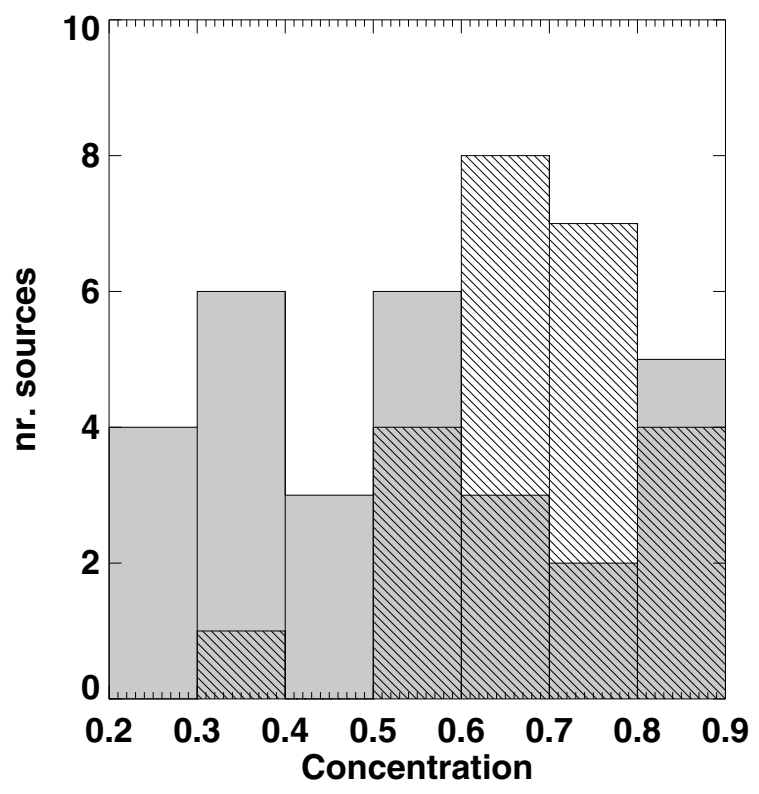

Fig. 13. The distribution of concentration of SCUBA (gray) and $\mathrm{HCO}^{+} 4-3$ (striped). Only sources with spatially resolved profiles have been taken into account. Note that the total number of sources is greater for SCUBA than for $\mathrm{HCO}^{+}$.

in Table 1 of Evans (1999). Thus, LFAM 26 is embedded in a high-density ridge.

Table 6 shows the results of gaussian fitting at other positions where multiple peaks in the $\mathrm{C}^{18} \mathrm{O} 3-2$ profiles were found. In the WL 19 field, south of the Elias 29 position, the same four components are again detected, although the $5.0 \mathrm{~km} \mathrm{~s}^{-1}$ layer is only seen north of WL 19. In the IRS 44 field along the same ridge to the south-west, the three layers at $2.6,3.5$ and $4.5 \mathrm{~km} \mathrm{~s}^{-1}$ are found.

Three layers are found in the Oph-B2 core, which contains the IRS 48 and IRS 37 sources. The first layer is at $2.8 \mathrm{~km} \mathrm{~s}^{-1}$, the second at $3.5 \mathrm{~km} \mathrm{~s}^{-1}$ and the third at $4.2 \mathrm{~km} \mathrm{~s}^{-1}$. Outside these two regions, spectra of $\mathrm{C}^{18} \mathrm{O}$ can be fitted with a single gaussian, almost always at $\sim 3.6 \mathrm{~km} \mathrm{~s}^{-1}$. The spatial variation in this layer seems to be minor compared with the other components and it is thus the most likely candidate for the foreground component with an extinction of $A_{\mathrm{v}} \sim 5.5 \mathrm{mag}$, as suggested by Dickman \& Herbst (1990). IRS 63, located far from the L 1688 core is at $2.8 \mathrm{~km} \mathrm{~s}^{-1}$, the same velocity as one of the layers in both Oph-B2 and Ophiuchus ridge region.

\subsection{Gas column density}

The total column density, $N_{\mathrm{H}_{2}}$, toward each source was calculated from the integrated intensity of the $\mathrm{C}^{18} \mathrm{O} 3-2$ line, $\int T_{\mathrm{MB}} \mathrm{d} V$, using the following formula:

$N\left(\mathrm{H}_{2}\right)=X \times 0.75 \times 10^{14} \frac{\mathrm{e}^{(16.6 / T)} \int T_{\mathrm{MB}} \mathrm{d} V}{1-\mathrm{e}^{(-33.2 / T)}}$

with $X$, the abundance of $\mathrm{H}_{2}$ with respect to $\mathrm{C}^{18} \mathrm{O}$, assumed to be $5.6 \times 10^{6}$ (Wilson \& Rood 1994) and $T$, the temperature of the cloud material, assumed to be $30 \mathrm{~K}$. The temperature is chosen to be higher than that of the surrounding cloud material, $\sim 15 \mathrm{~K}$, and reflects the warmer gas associated with the YSO where $\mathrm{CO}$ is not frozen out. This formula assumes the molecular excitation to be in LTE and isothermal. The $\mathrm{C}^{18} \mathrm{O} 3-2$ line is assumed to be completely optically thin. The resulting column densities can be found in Table 5, Col. 3. They range from $3.7 \times$ $10^{22} \mathrm{~cm}^{-2}$ for IRS 63 and the Oph F core to $31.6 \times 10^{22} \mathrm{~cm}^{-2}$ in the Oph A core. These correspond to visual extinctions $A_{\mathrm{V}}$ of 40-400 mag, assuming $N_{\mathrm{H}} / A_{\mathrm{V}}=1.8 \times 10^{21} \mathrm{~cm}^{-2} \mathrm{mag}^{-1}$ (Rachford et al. 2002) and $N_{\mathrm{H}} \approx 2 N\left(\mathrm{H}_{2}\right)$ (all hydrogen in molecular form). This range of column densities is similar to that found by Motte et al. (1998) and Stanke et al. (2006) using $1.3 \mathrm{~mm}$ continuum imaging, as well as the column densities found from the $850 \mu \mathrm{m}$ dust, see Sect. 5.4.

\subsection{Dust}

Table 5 includes the properties that can be derived from the continuum emission. The dust column density was calculated with the following equation

$N\left(\mathrm{H}_{2}\right)=\frac{S_{850}}{\Omega \mu m_{\mathrm{H}} \kappa_{850} B_{850}\left(T_{\mathrm{d}}\right)}$

where $S_{850}$ is the flux in the central beam, $\Omega$ is the main beam solid angle of $15^{\prime \prime}, \mu=2.33$ the mean molecular weight, $m_{\mathrm{H}}$ the mass of atomic hydrogen, $\kappa_{850}$ the dust opacity per gram of gas and dust at $850 \mu \mathrm{m}$ and $B_{850}(T)$ the Planck function at $850 \mu \mathrm{m}$ for a temperature $T$, assumed to be $30 \mathrm{~K}$. A $\kappa_{850}=0.01 \mathrm{~cm}^{2} \mathrm{gr}^{-1}$ is adopted from Ossenkopf \& Henning (1994) for dust with thin ice mantles (Table 1, Col. 4).

Two assumptions affect the comparison between the column densities derived from $\mathrm{C}^{18} \mathrm{O}$ and those from dust continuum. $\mathrm{C}^{18} \mathrm{O}$ can be frozen out onto the dust grains below $\sim 30 \mathrm{~K}$ at sufficiently high densities, so that $\mathrm{C}^{18} \mathrm{O}$ column densities are expected to be lower than those obtained from dust data. Second, all dust emission is assumed to originate in envelope or cloud material, whereas for several sources the disk contributes significantly to the continuum. These combined effects likely eplain the lack of correlation seen in Fig. 17 (left).

If one assumes that all emission detected at $850 \mu \mathrm{m}$ is associated with an isothermal envelope, a source mass can be determined following Eq. (4) from Shirley et al. (2000):

$$
\begin{aligned}
M_{D}= & \frac{S_{850} D^{2}}{B_{v}\left(T_{d}\right) \kappa_{v}}=3.69 \times 10^{-6} M_{\odot} \\
& \times S_{850}(\mathrm{Jy}) D^{2}(\mathrm{pc}) \times\left(\mathrm{e}^{16.9 / T_{\mathrm{d}}}-1\right) .
\end{aligned}
$$

Since the $3 \sigma$ sensitivity of the COMPLETE map is $80 \mathrm{mJy}$ within a $15^{\prime \prime}$ beam, the lower limit to the detectable mass is $0.04 M_{\odot}$. Such a low mass implies either a source that has nearly shed its envelope and is close to the pre-main sequence phase or an intrinsically very low luminosity object (Dunham et al. 2008).

Figure 17 shows the relation between the different column densities, envelope masses and the presence of dense gas as traced by $\mathrm{HCO}^{+}$. These figures clearly show that at higher envelope masses, the amount of dense gas and the total envelope mass correlate. The third figure illustrates that nearly all sources with centrally concentrated $\mathrm{HCO}^{+}$emission have larger envelope masses. Above an envelope mass of $0.1 M_{\odot}$, only a single source that is classified as confused in Sect. 6 (indicated with diamonds) is seen with $\mathrm{HCO}^{+}>2 \mathrm{~K} \mathrm{~km} \mathrm{~s}^{-1}$. This is CRBR $2422.8-$ 3423 , which is located in the line of sight with the envelope of IRS 43. The high mass found for CRBR 2422.8-3423 can be fully attributed to this envelope. 

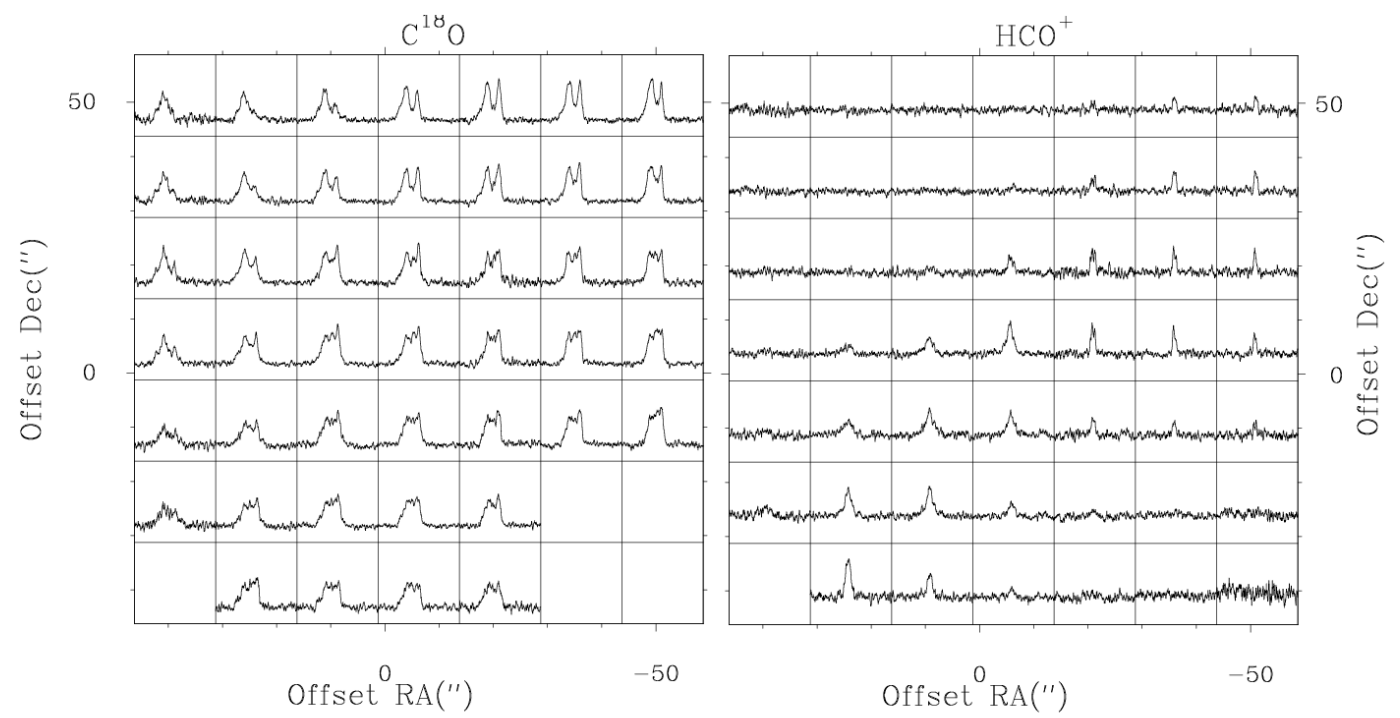

Fig. 14. The spectra of $\mathrm{C}^{18} \mathrm{O} 3-2$ and $\mathrm{HCO}^{+} 4-3$ of the LFAM 26 field. The intensity scale is -1 to $6 \mathrm{~K}$ in $T_{\mathrm{MB}}$ for $\mathrm{C}^{18} \mathrm{O}$ and -1 to $3 \mathrm{~K}$ for $\mathrm{HCO}^{+}$. OphE-MM3 is located at $\triangle \mathrm{RA}=+5^{\prime \prime}$ and $\Delta \mathrm{Dec}=-40^{\prime \prime}$ in this field and has no associated $\mathrm{HCO}^{+}$emission, except from the extended cloud.

\subsection{Effect of reddening on $\alpha_{I R}$}

Foreground material reddens the YSO and steepens the spectral slope $\alpha_{2-24 \mu \mathrm{m}}$. An estimate can be made of the foreground column density based on our $\mathrm{C}^{18} \mathrm{O}$ maps, by assuming that the average column or $A_{\mathrm{V}}$ at $45^{\prime \prime}$ offset from the source position in all directions originates entirely from this cloud. Although it is not known a priori whether this material is foreground, background, or a different YSO, we assume here that it is all foreground (see also Sect. 5.2) which provides an upper limit to the reddening. Note that the column densities at these positions were calculated with a temperature of $15 \mathrm{~K}$, a temperature associated with the surrounding cloud, instead of $30 \mathrm{~K}$, a temperature associated with protostellar envelopes. For typical dust opacities and the $A_{\mathrm{V}}=5.5 \mathrm{mag}$ layer as suggested by Dickman \& Herbst (1990), $\alpha_{2-24 \mu \mathrm{m}}$ is only increased by 0.18 (see also Crapsi et al. 2008). However, for higher $A_{\mathrm{V}}$ of $\sim 40 \mathrm{mag}$, as commonly found in the Ophiuchus ridge, this correction can be as high as 1.1, enough to change the classification of a Class II T Tauri star with disk to an embedded Class I source. Table 5 includes the (maximum) change in $\alpha_{2-24} \mu \mathrm{m}$ due to foreground reddening for each source derived by this method. Chapman et al. (2008) probe the $A_{K}$ in the northern part of Ophiuchus, including the northern part of the Oph-A area, where $A_{\mathrm{V}}$ values of $40 \mathrm{mag}$. and higher are found. In the Oph-A, Oph-B2 and Ridge regions, $A_{\mathrm{V}}$ of this magnitude were found from $\mathrm{C}^{18} \mathrm{O}$ column densities, reinforcing the conclusion that a strong extinction affects these regions on scales of only a few arcminutes, a resolution often not reached by extinction studies (Cambrésy 1999). Although these numbers have large uncertainties, they do illustrate the point that this potential extreme foreground reddening can lead to incorrect classifications for a significant number of sources in Ophiuchus.

An alternative is to use the velocity resolved $\mathrm{C}^{18} \mathrm{O}$ spectrum at the source position but subtract the layer that includes the source. For example, LFAM $26\left(\Delta \alpha_{\mathrm{IR}}=4.4\right)$, resides in a dense ridge (see Sect. 5.2). Subtracting the gaussian profile of this layer at $\sim 4.4 \mathrm{~km} \mathrm{~s}^{-1}$ gives an integrated intensity of $2.9 \mathrm{~K} \mathrm{~km} \mathrm{~s}^{-1}$, which corresponds to a column density of $10.7 \times 10^{22} \mathrm{~cm}^{-2}$, an $A_{\mathrm{V}}$ of $\sim 60 \mathrm{mag}$ and $\Delta \alpha_{\mathrm{IR}}=3.2$. If one assumes that the layers are arranged in distance in order of increasing $V_{\mathrm{LSR}}$, only the front layer at $3.5 \mathrm{~km} \mathrm{~s}^{-1}$ will redden and a column density of

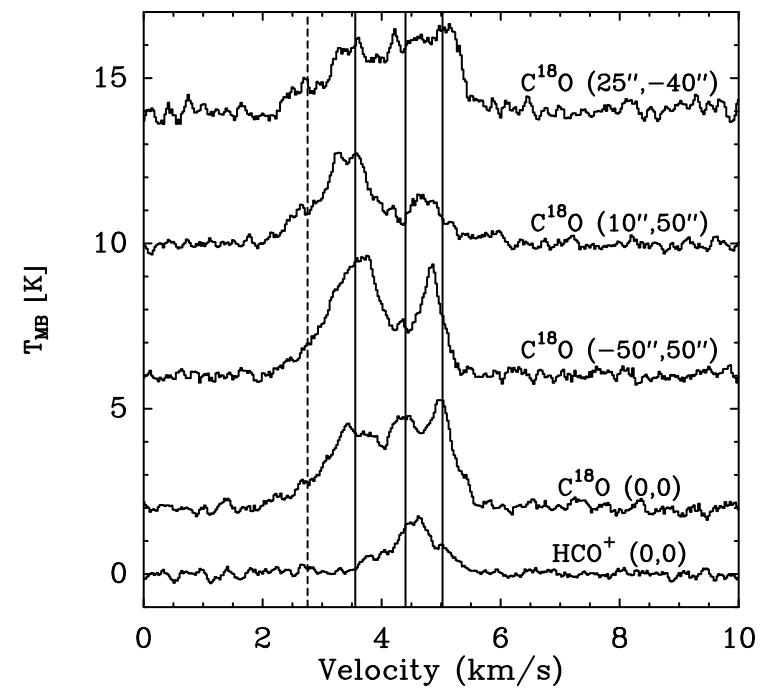

Fig. 15. Individual spectra at offset positions from LFAM 26 for $\mathrm{HCO}^{+}$ $\left(0^{\prime \prime}, 0^{\prime \prime}\right)$ and $\mathrm{C}^{18} \mathrm{O}(0,0),(-50,50),(10,50)$ and $(25,-40)$. The three main components at $3.5,4.4$ and $5.0 \mathrm{~km} \mathrm{~s}^{-1}$ are shown with solid lines. The component at $2.7 \mathrm{~km} \mathrm{~s}^{-1}$ identified by Boogert et al. (2002) is shown with a dashed line and is generally weaker or absent. The main component of the $\mathrm{HCO}^{+}$emission is located between the components at 4.2 and $4.9 \mathrm{~km} \mathrm{~s}^{-1}$ and peaks at $4.6 \mathrm{~km} \mathrm{~s}^{-1}$.

$6.6 \times 10^{22} \mathrm{~cm}^{-2}$, an $A_{\mathrm{V}}$ of $\sim 40$ and $\Delta \alpha_{\mathrm{IR}}=1.9$ are found. Thus, $\Delta \alpha_{\mathrm{IR}}$ is likely to be in between the maximum value listed in Table 5 and $\Delta \alpha_{\text {IR }} / 2$, although exceptional cases, such as OphEMM3, an edge-on disk in front of the cloud, do exist.

\subsection{SEDs: $L_{B o l}$ and $T_{B o l}$}

The IR and submillimeter fluxes have also been used to calculate the bolometric luminosity, $L_{\mathrm{bol}}$, and temperature, $T_{\mathrm{bol}}$, of each source. These numbers were calculated using the prismodial or the midpoint methods, with the inclusion of the 2MASS, IRAC, MIPS-24, MIPS-70, SHARC-II, SCUBA and $1.3 \mathrm{~mm}$ fluxes. Only fluxes with a $S / N>5$ were included. For a more thorough 
Table 6. The number and properties of the (foreground) layers for the fields of LFAM 26, IRS 44 and WL 19 in the Ophiuchus ridge and IRS 37 and IRS 4, located near Oph-B cores, see Fig. 7.

\begin{tabular}{llllll}
\hline \hline Field & Position & Nr. comp & $V_{\mathrm{LSR}}\left(\mathrm{km} \mathrm{s}^{-1}\right)$ & $\mathrm{C}^{18} \mathrm{O} \int T_{\mathrm{MB}} \mathrm{d} V\left(\mathrm{~K} \mathrm{~km} \mathrm{~s}^{-1}\right)$ & $\Delta V\left(\mathrm{~km} \mathrm{~s}^{-1}\right)$ \\
\hline LFAM 26 & 0,0 & 3 & $3.5,4.4,5.0$ & $1.8,0.9,1.1$ & $0.8,0.7,0.8$ \\
& $-20,+35$ & 4 & $2.7,3.5,4.4,5.0$ & $0.6,1.5,0.8,1.1$ & $0.9,0.7,0.7,0.7$ \\
\multirow{2}{*}{ IRS 44 } & $+35,-22$ & 3 & $3.5,4.4,5.0$ & $1.3,0.4,0.5,1.2,0.7,0.5$ & \\
& $0,0^{a}$ & 3 & $2.6,3.5,4.5$ & $0.5,2.3,2.3$ & $1.9,1.1,0.8$ \\
& $+20,+20$ (IRS 46) & 2 & $3.5,4.3$ & $1.9,1.3$ & $0.6,0.5$ \\
IRS 48 & $-50,20$ & 2 & $3.3,4.0$ & $1.1,2.5$ & $0.4,1.1$ \\
& 0,0 & 3 & $2.8,3.5,4.1$ & $0.6,1.7,2.6$ & $0.6,0.4,0.5$ \\
& $-36,+36$ & 3 & $2.9,3.5,4.1$ & $0.8,2.1,4.3$ & $0.4,0.4,0.5$ \\
WL 19 & $+38,6$ & 3 & $2.8,3.5,4.1$ & $0.4,1.1,3.5$ & $0.3,0.5,0.5$ \\
& 0,0 & 2 & $3.3,4.6$ & $2.7,4.7$ & $0.8,0.8$ \\
& $-6,36$ & 2 & $3.4,4.6$ & $2.8,5.6,1.1,0.8$ & \\
& $-20,-38$ & 2 & $3.2,4.2$ & $1.9,3.0$ & $0.7,1.0$ \\
IRS 37 & $-50,50$ & 4 & $2.6,3.5,4.3,5.1$ & $1.4,3.3,3.5,3.2$ & $0.5,0.6,0.7,0.7$ \\
& 0,0 & 2 & $3.0,4.4$ & $2.3,5.6$ & $0.7,1.1$ \\
& $+23,+7$ (WL3) & 2 & $3.2,4.4$ & $2.7,5.4$ & $0.8,1.0$ \\
& $-36,21$ & 3 & $3.0,3.6,4.2$ & $2.4,0.8,2.8$ & $0.9,0.2,0.8$ \\
\hline
\end{tabular}

${ }^{a}$ An outflow is detected at 0,0 in $\mathrm{HCO}^{+} 4-3$ which is probably the component seen at $2.6 \mathrm{~km} \mathrm{~s}^{-1}$.

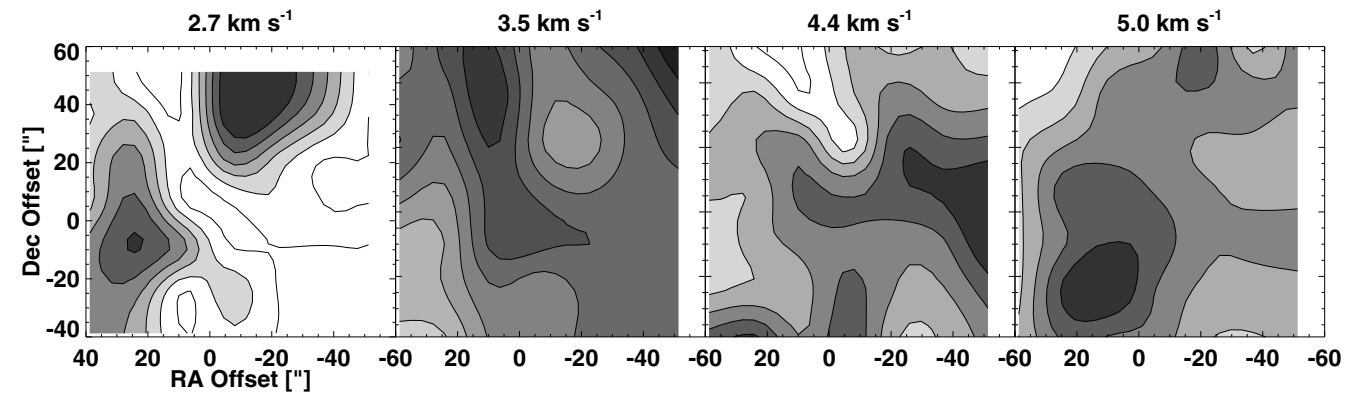

Fig. 16. The spatial distribution of the integrated gaussians fitted to the $\mathrm{C}^{18} \mathrm{O} 3-2$ spectra for each of the four layers seen in the field of LFAM 26.
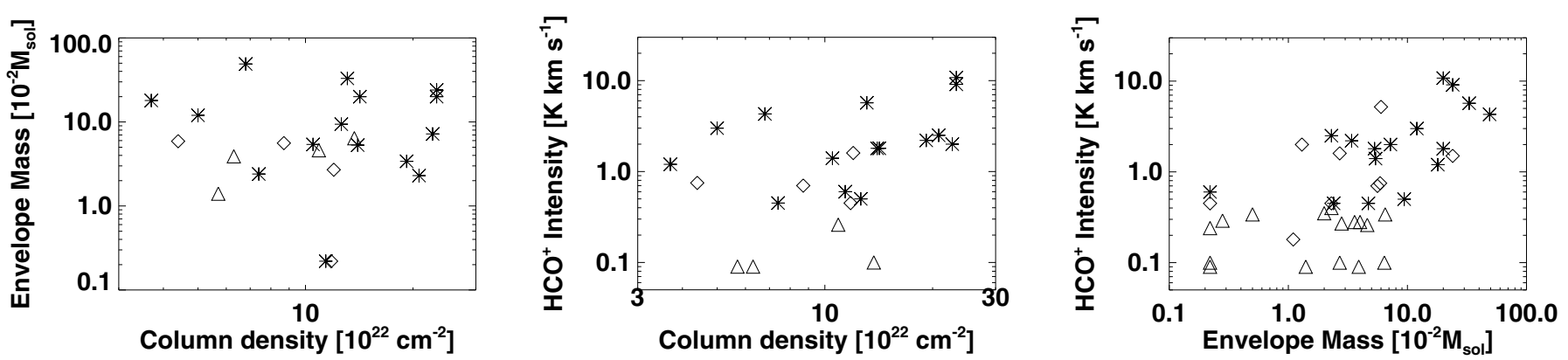

Fig. 17. Left: the gas column density (in $10^{22} \mathrm{~cm}^{-2}$ ) derived from $\mathrm{C}^{18} \mathrm{O} 3-2 \mathrm{vs.}$ the envelope mass (in $10^{-2} M_{\odot}$ ), derived from the SCUBA maps; middle: the column density vs. the integrated intensity of $\mathrm{HCO}^{+} 4-3$ in $\mathrm{K} \mathrm{km} \mathrm{s}^{-1}$ and right: the integrated intensity of $\mathrm{HCO}^{+} 4-3$ vs. the envelope mass. In all figures, sources with a centrally condensed $\mathrm{HCO}^{+}$emission are shown with stars, sources with an upper limit on $\mathrm{HCO}^{+} 4-3$ with triangles, and sources with $\mathrm{HCO}^{+}$emission not peaking on the source with diamonds.

discussion on the usage of these two methods, see Dunham et al. (in prep.) and Enoch et al. (2008).

Table 7 compares the values found for $T_{\text {bol }}$ and $L_{\text {bol }}$ in our work with those of Evans et al. (2009) for several sources. The differences stem from the fact that Evans et al. (2009) use CSO-Bolocam 1.1 millimeter data in a $30^{\prime \prime}$ beam, together with 2MASS, IRAC, and MIPS (24, 70 and $160 \mu \mathrm{m})$ fluxes. No SHARC-II or SCUBA fluxes were included, nor were sources with no detection at $1.1 \mathrm{~mm}$. As can be seen from the results for Elias 29 and GSS 30 IRS1, the inclusion of Bolocam and MIPS-160 produces a higher $L_{\mathrm{bol}}$ of up to a factor of 6 . In addition, $T_{\text {bol }}$ is consistently lower for the brighter sources. This can be largely attributed to the contributions from the surrounding cloud, which add significantly to the MIPS-160 and Bolocam fluxes. Although cloud emission is present in the IRAM-30 m $1.3 \mathrm{~mm}$ and SCUBA- $850 \mu \mathrm{m}$ observations used in our studies, the higher resolution of $15^{\prime \prime}$ limits such contributions. The exclusion of MIPS-160 in our work causes the $L_{\text {bol }}$ to be 
Table 7. Comparison between $L_{\mathrm{bol}}$ and $T_{\mathrm{bol}}$ as calculated in Evans et al. (2009) (indicated with Evans) and this work (indicated with van K.).

\begin{tabular}{lllll}
\hline \hline Source & $\begin{array}{l}L_{\text {bol }} \\
\text { Evans } \\
\left(L_{\odot}\right)\end{array}$ & $\begin{array}{l}L_{\text {bol }} \\
\text { van K. } \\
\left(L_{\odot}\right)\end{array}$ & $\begin{array}{l}T_{\text {bol }} \\
\text { Evans } \\
(\mathrm{K})\end{array}$ & $\begin{array}{l}T_{\text {bol }} \\
\text { van K. } \\
(\mathrm{K})\end{array}$ \\
\hline GSS 30 & 8.7 & 3.3 & 150 & 123 \\
CRBR 2339.1 & $<0.065$ & 0.055 & $<370$ & 426 \\
WL 2 & 0.12 & 0.09 & 428 & 573 \\
LFAM 26 & 0.15 & 0.044 & 110 & 238 \\
WL 17 & 0.6 & 0.67 & 310 & 323 \\
Elias 29 & 17.9 & 2.5 & 257 & 424 \\
IRS 42 & 1.2 & 1.4 & 600 & 540 \\
CRBR 2422.8 & 0.19 & 0.44 & $<300$ & 157 \\
IRS 43 & 3.8 & 1.0 & 160 & 134 \\
IRS 44 & 15 & 1.1 & 110 & 140 \\
\hline
\end{tabular}

underestimated and the $T_{\text {bol }}$ to be overestimated, due to the lack of a point at far-IR wavelengths near the peak of the SED. Isolated, unresolved sources such as IRS 42 show little to no differences between the two studies, confirming the influence of the environment. Future high resolution $\left(<10^{\prime \prime}\right)$ observations with the PACS instrument on Herschel covering the 60 to $600 \mu \mathrm{m}$ window will be able to fully constrain the far-IR emission and SED.

\subsection{Ice and silicate absorption}

In Fig. 12, the IRS spectra for 28 of the sources in our sample are plotted. 17 sources show ice absorption bands at $15.2 \mu \mathrm{m}$ due to $\mathrm{CO}_{2}$ ice (see Table 8), and 11 do not (WL 3, LFAM 26, VSSG 1, CRBR 2317.3, CRBR 2315.8, IRS 48, CRBR 2339.1, SR 21, Haro 1-4, IRS 46 and SR 9). A few of the known edge-on disks, such as CRBR 2422.8-3423.2, show ice absorptions.

Crapsi et al. (2008) modelled the spectral features at $3 \mu \mathrm{m}$ $\left(\mathrm{H}_{2} \mathrm{O}\right.$ ice), $10 \mu \mathrm{m}$ (silicate) and $15.2 \mu \mathrm{m}\left(\mathrm{CO}_{2}\right.$ ice) for a large grid of models, including embedded sources and $\mathrm{T}$ Tauri disks, both seen at a wide variety of angles. The ice absorptions at 3 and $15.2 \mu \mathrm{m}$ were found to be more prevalent for embedded sources. However, edge-on disks can also show such absorptions in almost equal strength. The relation of the $3 \mu \mathrm{m}$ ice absorptions with the envelope mass of both embedded and disk sources is given in Fig. 4 of Crapsi et al. (2008) and a similar relation was found for the $15.2 \mu \mathrm{m}$ band. Cold foreground clouds can also contribute significantly.

Using the determination of the column densities from the $\mathrm{C}^{18} \mathrm{O}$ and SCUBA maps in Sects. 5.3 and 5.4, Eqs. (3) and (4), the ice absorption can be compared to the amount of reddening as indicated by $\Delta \alpha_{\mathrm{IR}}$. Although ice absorptions are more commonly found in sources with a higher reddening, it is concluded that the YSOs must be characterized on a source by source basis to locate the origin of the ices. The disk source CRBR 2422.8 has deep ice absorptions (Pontoppidan et al. 2005), most likely originating the large column of foreground material $(\Delta \alpha=0.9)$. However, LFAM 26, which has an even higher reddening $(\Delta \alpha=$ 1.7 ), shows no sign of ice absorption. Of course, it is possible LFAM 26 is located in front of the cloud and as such the presence or absence of ice absorption could serve as a useful diagnostic of the geometry of the YSO-cloud system. The silicate feature around $10 \mu \mathrm{m}$ is detected for fewer sources than the 15.2 $\mathrm{CO}_{2}$ ice absorption. Although LFAM 26, WL17, Elias 29, IRS 42, WL 3, IRS 54, CRBR 2315.8-1700, CRBR 2339.12032 and CRBR 2422.8-3423 all have silicate absorption, many other sources were not observed at the wavelengths of the silicate feature.

\section{Classification}

\subsection{Physical classification}

A new classification based on physical parameters has gradually been introduced (Whitney et al. 2003b,a; Robitaille et al. 2006) but is not yet as commonly in use as the traditional Class system. This new classification identifies the evolutionary stage using the physical characteristics as opposed to the observed characteristics. The different evolutionary stages are determined from the ratios between $M_{\text {disk }}, M_{\text {envelope }}$ and $M_{\text {star }}$. The total circumstellar mass, $M_{\text {circum }}$, is defined as $M_{\text {disk }}+M_{\text {envelope }}$. The evolutionary stages of this classification are:

- stage 0 , deeply embedded sources with $M_{\text {disk }} / M_{\text {envelope }} \ll 1$ and $M_{\text {circum }} / M_{\text {star }} \sim 1$;

- stage 1 , embedded sources with $0.1<M_{\text {disk }} / M_{\text {envelope }}<2$ and $M_{\text {circum }}<M_{\text {star }}$;

- stage 2, classical T Tauri stars with gas-rich disks $\left(M_{\text {envelope }}=0\right.$ and $\left.M_{\text {disk }} / M_{\text {star }} \ll 1\right)$;

- stage 3, pre-main sequence stars with tenuous disks.

Stage 0 sources are equivalent to Class 0 sources and can be identified by their submm characteristics as originally put forward by André et al. (1993). Stage 2 and stage 3 sources, corresponding to the classical $\mathrm{T}$ Tauri stars with disks and pre-main sequence stars with (gas-poor) tenuous disks, are identified using the IR excess combined with optical/near-IR spectroscopic properties. The amount of IR excess and the wavelength where this excess starts are often used to distinguish stage 2 and 3 sources.

The Stage 1 sources are most problematic to uniquely identify based on observational characteristics. Both Whitney et al. (2003a) and Crapsi et al. (2008) show that the traditional identification methods of using the IR spectral slope $\alpha_{\mathrm{IR}}$ and $T_{\mathrm{bol}}$ are insufficient to distinguish the edge-on disks from embedded sources, as well as missing evolved, face-on embedded YSOs that are (mis)classified as Class II. One of the main parameters to unambiguously identify Stage 1 sources is the $M_{\text {disk }} / M_{\text {envelope }}$ ratio as determined from millimeter continuum observations in a large and small ( $\left.\sim 2^{\prime \prime}\right)$ beam (Crapsi et al. 2008). However, the interferometric observations needed to unambiguously constrain the disk masses are still time-consuming for large samples of sources.

\subsection{Identifying embedded stage 1 sources with molecular emission}

To uniquely identify embedded sources and distinguish them from edge-on disks, prestellar cores and possible background AGB stars and galaxies without having to resort to interferometers, we propose to use the single-dish molecular emission. Embedded sources will be bright and centrally peaked in $\mathrm{HCO}^{+} 4-3$ and $\mathrm{C}^{18} \mathrm{O} 3-2$. The $\mathrm{HCO}^{+}$line maps will detect all but the most tenuous envelopes. The concentration parameter shows that $\mathrm{HCO}^{+} 4-3$ is much more accurate in identifying protostellar envelopes than continuum dust emission. The $\mathrm{HCO}^{+}$maps also spatially resolve larger envelopes (e.g., the VLA 1623 and GSS 30 envelopes). The nearby environment, including foreground layers, is in turn characterized by the $\mathrm{C}^{18} \mathrm{O} 3-2$ (see Sects. 4 and 5).

To distinguish embedded sources from isolated edge-on disks, a good limit on the $\mathrm{HCO}^{+} 4-3$ emission is $T_{\mathrm{MB}}>0.4 \mathrm{~K}$. 
Thi et al. (2004) observed four well-studied gas-rich disk sources in a large range of molecular transitions, including $\mathrm{HCO}^{+} 4-3$, and found $T_{\mathrm{MB}} \sim 0.1 \mathrm{~K}$, four times below our adopted limit. Although disks around embedded sources could be somewhat larger and more massive, it is assumed that the contribution from even the largest embedded disks does not exceed our adopted limit. This value is naturally small because of the severe dilution of emission originating in a disk within the $15^{\prime \prime}$ JCMT beam. For example, a $20 \mathrm{~K}$ optically thick line from a $2^{\prime \prime}$ disk would be diluted to $0.35 \mathrm{~K}$. Indeed, for two embedded sources for which interferometric $\mathrm{HCO}^{+}$data exist (IRS 63 and Elias 29, Lommen et al. 2008), the $\mathrm{HCO}^{+}$contribution of the disk does not exceed this limit (see Sect. 6.3). The following definitions were therefore used to classify the sample:

\section{- Stage 1}

- extended $\mathrm{HCO}^{+}$emission, peaking on source, with $C_{\mathrm{HCO}^{+}}>0.7$ and $\int T_{\mathrm{MB}} \mathrm{d} V>0.4 \mathrm{~K} \mathrm{~km} \mathrm{~s}^{-1}$ and/or;

- an $\mathrm{HCO}^{+}$profile that is not extended, but $\int T_{\mathrm{MB}} \mathrm{d} V>$ $0.4 \mathrm{~K} \mathrm{~km} \mathrm{~s}^{-1}$, with SCUBA both extended and $C_{850}>$ 0.7 ;

- ice absorptions are usually prominent.

- Stage 2

- absence of $\mathrm{HCO}^{+}$, SCUBA and $\mathrm{C}^{18} \mathrm{O}$ down to the rms limits at the IR position or;

- no variation of $\mathrm{HCO}^{+}$, SCUBA and $\mathrm{C}^{18} \mathrm{O}$ on scales of $30^{\prime \prime}(C<0.4)$ or;

- no $\mathrm{C}^{18} \mathrm{O} 3-2$ or SCUBA is seen at scales larger than the central beam of $15^{\prime \prime}$ with $\mathrm{HCO}^{+} 4-3$ not extended and $<0.4 \mathrm{~K} \mathrm{~km} \mathrm{~s}^{-1}$ or;

$-\alpha_{\mathrm{IR}}<-0.5$.

- Confused

- $\mathrm{HCO}^{+}$, SCUBA or $\mathrm{C}^{18} \mathrm{O}$ peaking more than $20^{\prime \prime}$ away from the IR position.

In the rare case that an embedded source is viewed face-on (into the outflow cone, see Whitney et al. (2003b)), the above restrictions will identify such a source as stage 1 , having $\alpha_{\mathrm{IR}}<$ -0.3 , but with extended $\mathrm{HCO}^{+}$emission, peaking on source $\left(\int T_{\mathrm{MB}} \mathrm{d} V>0.4 \mathrm{~K} \mathrm{~km} \mathrm{~s}^{-1}\right)$.

Table 8 shows the results for this classification. In Appendix the sources and their classifications are discussed on a source by source basis. Sixteen Class I sources were identified as embedded Stage 1 sources with envelopes varying in mass and size. The sources GSS 30 IRS 1 and LFAM 1 appear to be embedded within an approximately spherical envelope encompassing both sources. The sources IRS 37 and WL 3 are embedded in a highly non-spherical envelope covering both sources. In addition to these 16 sources, VLA 1623 is classified as embedded, but considered a Stage 0 source due to its high sub-mm flux relative to the total luminosity. From comparison with Jørgensen et al. (2008) (see Sect. 6.5), we can conclude that all embedded sources were found down to a mass limit (envelope+disk) of $0.04 M_{\odot}$. Deep searches for even lower luminosity sources have found only two likely candidate sources within our field (Dunham et al. 2008). Deep (interferometric) sub-mm observations are needed to identify if such sources are truly embedded.

Five sources were found to be confused ( $\mathrm{C}$ in Table 8). The exact nature of these sources (obscured, disk or other) cannot be determined without interferometric observations. However, molecular emission rules out a protostellar envelope. Observations of $\mathrm{C}^{18} \mathrm{O}$ show that cloud material is located in the line of sight towards these sources. They will be further discussed in Sect. 6.4.
Twenty-three sources were found to be Stage 2 sources ( 2 in in Table 8). Of this sample, sources with $\alpha_{\mathrm{IR}}>0$ and with no correction from extinction (see Table 5) are likely to be edge-on or close to edge-on disks. Sources that do have a potential correction to $\alpha_{\mathrm{IR}}$, such as CRBR 2422.8 or IRS 48, do not have to be edge-on. All sources that were initially known to be disk sources are identified as Stage 2 sources using this method. If one considers that disk sources with $\alpha_{I R}>-0.3$, when corrected for the maximum $\Delta \alpha_{\mathrm{IR}}$ ), are edge-on, only 7 out of 23 Stage 2 sources of our sample are expected to be edge-on. The total amount of Class II sources found in Ophiuchus by c2d is 176, and although this includes L 1689, the bulk of these disks exist close to or are part of the L 1688 region (Evans et al. 2009). Thus, our number of edge-on disks appears to be only a small fraction of the total disk sample.

No face-on embedded sources were found that have $\alpha_{\mathrm{IR}}<$ -0.3 , but do show a $\mathrm{HCO}^{+}$spectrum that is both peaking on source and stronger than $0.4 \mathrm{~K} \mathrm{~km} \mathrm{~s}^{-1}$. This very low fraction is not surprising, since such sources have strong restrictions on the line of sight.

Using the results from Spitzer (Evans et al. 2007; Jørgensen et al. 2006; Evans et al. 2009; Allen et al.; in prep.) a comparison can be made between the classification using $\alpha_{\mathrm{IR}}$ and the new method. It is found that $\sim 50 \%$ (11 out of 22 ) of the sources classified as Class I are Stage 2 disks and not embedded. Note that this includes the known disks OphE MM3 and CRBR 2422.8. However, of the Flat spectrum sources, $\sim 50 \%$ (6 out of 13) appear to be embedded, while the other half is either confused or classified as a Stage 2 disk. In the end, the sample of embedded sources found by c2d changes from 22 out of 45 to 17 out of 45 .

\subsection{Late stage 1 sources}

Of the sixteen embedded Stage 1 sources, four sources (WL 6, WL 17, IRS 54 and IRS 63) were found to have only marginal envelopes left, with $\mathrm{C}^{18} \mathrm{O}$ and $850 \mu \mathrm{m}$ emission suggesting that at most a few $\times 0.01 M_{\odot}$ remains in the envelope. Lommen et al. (2008) observed IRS 63 using the Sub-Millimeter Array (SMA) and found that $50 \%$ of the continuum emission originates within a disk no bigger than $200 \mathrm{AU}$ in size with a mass of $0.055 M_{\odot}$ and the remainder in a remnant envelope with a mass of $0.058 M_{\odot} . \mathrm{HCO}^{+} 3-2$ was also detected with the SMA, originating mostly in the disk. When convolved with the JCMT beam, the observed brightness is $0.28 \mathrm{~K} \mathrm{~km} \mathrm{~s}^{-1}$. The $\mathrm{HCO}^{+} 4-3$ single-dish intensity as observed with HARP-B is $0.75 \mathrm{~K} \mathrm{~km} \mathrm{~s}^{-1}, 3$ times as bright. For typical disk excitation conditions, $\mathrm{HCO}^{+} 4-3$ is expected to be equal or weaker compared to the 3-2. It can thus be concluded from the much higher observed intensity in the 4-3 line, that at least half of the 4-3 emission within the JCMT beam likely originates in the protostellar envelope and would not be seen by the SMA.

In all four sources, little to no extended $\mathrm{C}^{18} \mathrm{O}$ is seen. The distributions and strengths in the $\mathrm{HCO}^{+}$and $\mathrm{C}^{18} \mathrm{O}$ lines of the other three sources are similar to that of IRS 63, but the SCUBA image of IRS 63 is a factor of 4 brighter. For IRS 54, the envelope is spatially resolved in $\mathrm{HCO}^{+}, \mathrm{C}^{18} \mathrm{O}$ and SCUBA.

Although rare, it is concluded that these sources are Stage 1 sources in transition to a Stage 2 source (marked with 1(T) in Table 8). They have accreted or dispersed almost their entire envelope. Continuum emission in the sub-mm is likely to contain a large contribution from the protostellar disk. 
Table 8. The classification of the source sample with the recipe outlined in the text.

\begin{tabular}{|c|c|c|}
\hline Source & Code $^{a}$ & Stage \\
\hline \multicolumn{3}{|c|}{ Embedded sources } \\
\hline GSS 30 & $\mathrm{~S}_{\mathrm{HC} 8} \mathrm{E}_{\mathrm{H} 8} \mathrm{P}_{\mathrm{H} 8} \mathrm{I}$ & 1 \\
\hline LFAM 1 & $\mathrm{~S}_{\mathrm{H} 8} \mathrm{~N}_{\mathrm{C}} \mathrm{E}_{\mathrm{H} 8} \mathrm{P}_{\mathrm{H}} \mathrm{O}_{8}$ & 1 \\
\hline VLA 1623 & $\mathrm{~S}_{\mathrm{HC} 8} \mathrm{E}_{\mathrm{H} 8} \mathrm{P}_{\mathrm{H} 8}$ & 0 \\
\hline WL 12 & $\mathrm{~S}_{\mathrm{HC} 8} \mathrm{E}_{\mathrm{HC} 8} \mathrm{P}_{\mathrm{HC} 8} \mathrm{I}$ & 1 \\
\hline LFAM 26 & $\mathrm{~S}_{\mathrm{HC} 8} \mathrm{E}_{\mathrm{HC} 8} \mathrm{P}_{\mathrm{H} 8} \mathrm{O}_{\mathrm{C}}$ & 1 \\
\hline WL 17 & $\mathrm{~S}_{\mathrm{H} 8} \mathrm{~N}_{\mathrm{C}} \mathrm{P}_{\mathrm{H} 8} \mathrm{I}$ & $1(\mathrm{~T})$ \\
\hline Elias 29 & $\mathrm{~S}_{\mathrm{HC} 8} \mathrm{E}_{\mathrm{H} 8} \mathrm{P}_{\mathrm{H} 8} \mathrm{I}$ & 1 \\
\hline IRS 37 & $\mathrm{~S}_{\mathrm{HC}} \mathrm{W}_{8} \mathrm{E}_{\mathrm{HC} 8} \mathrm{P}_{\mathrm{HC} 8} \mathrm{I}$ & 1 \\
\hline WL 3 & $\mathrm{~S}_{\mathrm{HC}} \mathrm{W}_{8} \mathrm{E}_{\mathrm{HC} 8} \mathrm{P}_{\mathrm{H}}$ & 1 \\
\hline WL 6 & $\mathrm{~S}_{\mathrm{HC}} \mathrm{N}_{8} \mathrm{I}$ & $1(\mathrm{~T})$ \\
\hline IRS 43 & $\mathrm{~S}_{\mathrm{HC} 8} \mathrm{E}_{\mathrm{H} 8} \mathrm{P}_{\mathrm{H} 8} \mathrm{I}$ & 1 \\
\hline IRS 44 & $\mathrm{~S}_{\mathrm{HC} 8} \mathrm{E}_{\mathrm{HC} 8} \mathrm{P}_{\mathrm{HC} 8}$ & 1 \\
\hline Elias 32 & $\mathrm{~S}_{\mathrm{HC} 8} \mathrm{E}_{\mathrm{H} 8} \mathrm{P}_{\mathrm{H} 8} \mathrm{I}$ & 1 \\
\hline Elias 33 & $\mathrm{~S}_{\mathrm{HC} 8} \mathrm{E}_{\mathrm{H} 8} \mathrm{P}_{8} \mathrm{I}$ & 1 \\
\hline IRS 54 & $\mathrm{~S}_{\mathrm{HC}} \mathrm{W}_{8} \mathrm{E}_{\mathrm{HC} 8} \mathrm{P}_{\mathrm{HC} 8} \mathrm{I}$ & $1(\mathrm{~T})$ \\
\hline IRAS 16285-2355 & $\mathrm{S}_{\mathrm{H} 8} \mathrm{~W}_{\mathrm{C}} \mathrm{E}_{\mathrm{HC} 8} \mathrm{P}_{\mathrm{HC} 8}$ & \\
\hline IRS 63 & $\mathrm{~S}_{\mathrm{H} 8} \mathrm{~W}_{\mathrm{C}} \mathrm{E}_{\mathrm{HC} 8} \mathrm{P}_{\mathrm{HC} 8} \mathrm{I}$ & $1(\mathrm{~T})$ \\
\hline \multicolumn{3}{|c|}{ Confused Sources } \\
\hline CRBR 2324.1-1619 & $\mathrm{S}_{\mathrm{HC} 8} \mathrm{E}_{\mathrm{HC} 8} \mathrm{O}_{\mathrm{HC} 8}$ & $\mathrm{C}$ \\
\hline GY51 & $\mathrm{S}_{8} \mathrm{~N}_{\mathrm{HC}} \mathrm{E}_{8} \mathrm{O}_{8}$ & $\mathrm{C}$ \\
\hline WL19 & $\mathrm{S}_{\mathrm{C} 8} \mathrm{~N}_{\mathrm{H}} \mathrm{E}_{\mathrm{HC} 8} \mathrm{O}_{\mathrm{HC} 8} \mathrm{I}$ & $\mathrm{C}$ \\
\hline IRS 42 & $\mathrm{~S}_{\mathrm{HC} 8} \mathrm{E}_{\mathrm{H} 8} \mathrm{O}_{\mathrm{H} 8} \mathrm{I}$ & $\mathrm{C}$ \\
\hline GY 256 & $\mathrm{~S}_{\mathrm{HC}} \mathrm{N}_{8}$ & $\mathrm{C}$ \\
\hline \multicolumn{3}{|c|}{ Disks } \\
\hline SSTc2d J162527.6-243648 & $\mathrm{N}_{\mathrm{HC} 8}$ & 2 \\
\hline GSS 26 & $\mathrm{~S}_{8} \mathrm{~N}_{\mathrm{HC}} \mathrm{P}_{8}$ & 2 \\
\hline CRBR 2315.8-1700 & $\mathrm{S}_{\mathrm{H} 8} \mathrm{~N}_{\mathrm{C}} \mathrm{E}_{\mathrm{H}} \mathrm{P}_{8} \mathrm{O}_{\mathrm{H}}$ & 2 \\
\hline CRBR 2317.3-1925 & $\mathrm{S}_{\mathrm{H}} \mathrm{W}_{8} \mathrm{~N}_{\mathrm{C}}$ & 2 \\
\hline VSSG 1 & $\mathrm{~W}_{8} \mathrm{~N}_{\mathrm{HC}} \mathrm{P}_{8}$ & 2 \\
\hline CRBR 2339.1-2032 & $\mathrm{S}_{\mathrm{H}} \mathrm{W}_{8} \mathrm{~N}_{\mathrm{C}} \mathrm{P}_{8}$ & 2 \\
\hline WL 2 & $\mathrm{~W}_{\mathrm{H} 8} \mathrm{~N}_{\mathrm{C}} \mathrm{E}_{\mathrm{H} 8} \mathrm{O}_{\mathrm{H} 8}$ & 2 \\
\hline GY 224 & $\mathrm{~W}_{8} \mathrm{~N}_{\mathrm{HC}} \mathrm{P}_{8} \mathrm{I}$ & 2 \\
\hline WL 20S & $\mathrm{S}_{\mathrm{C} 8} \mathrm{~N}_{\mathrm{H}} \mathrm{P}_{8} \mathrm{I}$ & 2 \\
\hline IRS 48 & $\mathrm{~W}_{\mathrm{C} 8} \mathrm{~N}_{\mathrm{H}} \mathrm{E}_{\mathrm{C}} \mathrm{P}_{8}$ & 2 \\
\hline GY 312 & $\mathrm{~N}_{\mathrm{HC} 8}$ & 2 \\
\hline IRS 51 & $\mathrm{~S}_{\mathrm{H} 8} \mathrm{~W}_{\mathrm{C}} \mathrm{E}_{\mathrm{HC} 8} \mathrm{P}_{8} \mathrm{O}_{\mathrm{HC}} \mathrm{I}$ & 2 \\
\hline SSTc2d J162741.6-244645 & $\mathrm{W}_{8} \mathrm{~N}_{\mathrm{HC}}$ & 2 \\
\hline SSTc2d J162748.2-244225 & $\mathrm{N}_{\mathrm{HC} 8}$ & 2 \\
\hline SSTc2d J162857.9-244055 & $\mathrm{S}_{8} \mathrm{~N}_{\mathrm{HC}}$ & 2 \\
\hline Haro 1-4 & $\mathrm{N}_{\mathrm{HC} 8}$ & 2 \\
\hline DoAR 25 & $\mathrm{~S}_{8} \mathrm{~N}_{\mathrm{HC}} \mathrm{P}_{8}$ & 2 \\
\hline OphE MM3 & $\mathrm{S}_{\mathrm{HC}} \mathrm{W}_{8} \mathrm{E}_{\mathrm{HC} 8} \mathrm{O}_{\mathrm{H} 8}$ & 2 \\
\hline SR 21 & $\mathrm{~W}_{8} \mathrm{~N}_{\mathrm{HC}} \mathrm{P}_{8}$ & 2 \\
\hline CRBR 2422.8-3423 & $\mathrm{S}_{\mathrm{H} 8} \mathrm{~N}_{\mathrm{C}} \mathrm{E}_{8} \mathrm{O}_{8} \mathrm{I}$ & 2 \\
\hline IRS 46 & $\mathrm{~S}_{8} \mathrm{~W}_{\mathrm{C}} \mathrm{N}_{\mathrm{H}} \mathrm{E}_{\mathrm{HC} 8} \mathrm{O}_{\mathrm{HC} 8}$ & 2 \\
\hline SR 9 & $\mathrm{~N}_{\mathrm{HC} 8}$ & 2 \\
\hline 2Mass 16282 & $\mathrm{~W}_{8} \mathrm{~N}_{\mathrm{HC}}$ & 2 \\
\hline
\end{tabular}

${ }^{a}$ The coding in column 2 is as follows: $\mathrm{S}, \mathrm{W}$ and $\mathrm{N}$ determine if a line or continuum is Strong, Weak or Not detected/observed. E, P and O determine if a source is Extended, Peaking or Offset peaking. The subscripts $\mathrm{H}, \mathrm{C}$ and 8 refer to the $\mathrm{HCO}^{+}, \mathrm{C}^{18} \mathrm{O}$ and $850 \mu \mathrm{m}$. I is detected $\mathrm{CO}_{2}$ ice absorption. Column 3 lists the classification. 1 is a stage $1 \mathrm{em}-$ bedded YSO; 2 is a disk and $\mathrm{C}$ is Confused.

\subsection{Confused sources}

For the five confused sources, CRBR 2324.1-1619, GY 51, WL 19, IRS 42 and GY 256, the exact nature cannot be determined using $\mathrm{HCO}^{+}$, except that such sources are ruled out as embedded YSOs. None of them have the characteristic peak of $\mathrm{HCO}^{+}$that most Stage 1 embedded YSOs have. However, at least two out of the three tracers, $\mathrm{HCO}^{+}, \mathrm{C}^{18} \mathrm{O}$ and SCUBA, are detected so a few possibilities remain. The first is an (edge-on)
Table 9. Comparison between the classification of YSOs using $\alpha_{\text {IR }}$ by Evans et al. (2009) and our method using molecular emission.

\begin{tabular}{lllll}
\hline \hline & Class I & Flat Spectrum & Class II & Total \\
\hline Stage 1 & 11 & 6 & 0 & 17 \\
Confused & 1 & 3 & 1 & 5 \\
Stage 2 & 10 & 4 & 9 & 23 \\
\hline Total & 22 & 13 & 10 & 45 \\
\hline
\end{tabular}

${ }^{a}$ Note that although it is possible for Flat Spectrum sources to be embedded, confused sources are ruled out to be embedded.

disk, in front of cloud material. This would be similar to the case of OphE-MM3, which was confirmed to be an edge-on disk in front of a dense core (Brandner et al. 2000). Without the near-IR imaging, OphE-MM3 would have been classified as confused. A second possibility is that they are background sources. The most likely options are then a $\mathrm{T}$ Tauri star with disk behind the cloud (possibly edge-on), or an AGB star. Background main sequence stars are identified within the c2d delivery document (Evans et al. 2007) and are thus highly unlikely to be included in our sample. Chances of background AGB stars or galaxies being aligned with the cloud are small, but according to Jørgensen et al. (2008), a single background source can be expected. A final possibility is that the sources are very late Stage 1 embedded YSOs, very close to the Stage 2 phase. An upper limit of only $0.02 M_{\odot}$ is found for their envelope mass.

\subsection{Comparison to other methods}

Figure 18 shows the effectiveness of the classification of sources using the method above, as compared to methods using $\alpha_{\mathrm{IR}}$ and $T_{\text {bol }}$. A key parameter is the strength of the $\mathrm{HCO}^{+}$integrated intensity. The following limits were adopted between embedded and disk sources: $\alpha_{\mathrm{IR}}>0, T_{\text {bol }}<650 \mathrm{~K}$ and $\mathrm{HCO}^{+}>$ $0.4 \mathrm{~K} \mathrm{~km} \mathrm{~s}^{-1}$.

The advantages of the method using molecular emission are immediately apparent. Although the classical methods of using $T_{\text {bol }}$ and $\alpha_{\text {IR }}$ are able to identify embedded sources, both methods also incorrectly identify a number of Stage 2 disks as embedded, especially for $L_{\mathrm{bol}}<1 L_{\odot}$. These are the edge-on disks. If one uses the limits of the integrated intensity for $\mathrm{HCO}^{+}$, isolated disk sources are easily identified. With the additional restriction of having a peak within the $\mathrm{HCO}^{+}$map, confused sources or sources in front of the cloud can be readily identified.

The use of ice absorption, as studied by Crapsi et al. (2008), is limited by similar constraints. Most embedded sources, with the exception of LFAM 26 and WL 3, show $\mathrm{CO}_{2}$ ice absorption at $15.2 \mu \mathrm{m}$. However, 4 disk sources IRS 51, WL 19, WL 20S and CRBR 2422.8-3423 also show strong ice absorptions. These ice absorptions are caused both by the foreground layers as discussed in Sect. 5.2 as well as the material in the disk itself, if viewed edge-on (as is the case for CRBR 2422.8). Thus, the presence of ice absorption cannot be used to unambiguously identify embedded sources from other sources. Even if the origin of the ice absorption can be attributed to a protostellar envelope, its strength does not seem to be an indication of evolution, e.g. IRS 63, a small envelope, has a deeper ice absorption than the large envelope of GSS 30.

Jørgensen et al. (2008) published a list of "candidate" embedded YSOs based on two criteria. First the colors of sources using the IRAC and MIPS results ([3.6]-[4.5] and [8]-[24]). Second, the proximity of MIPS sources to SCUBA cores. For these cores, the concentration is an important parameter. 

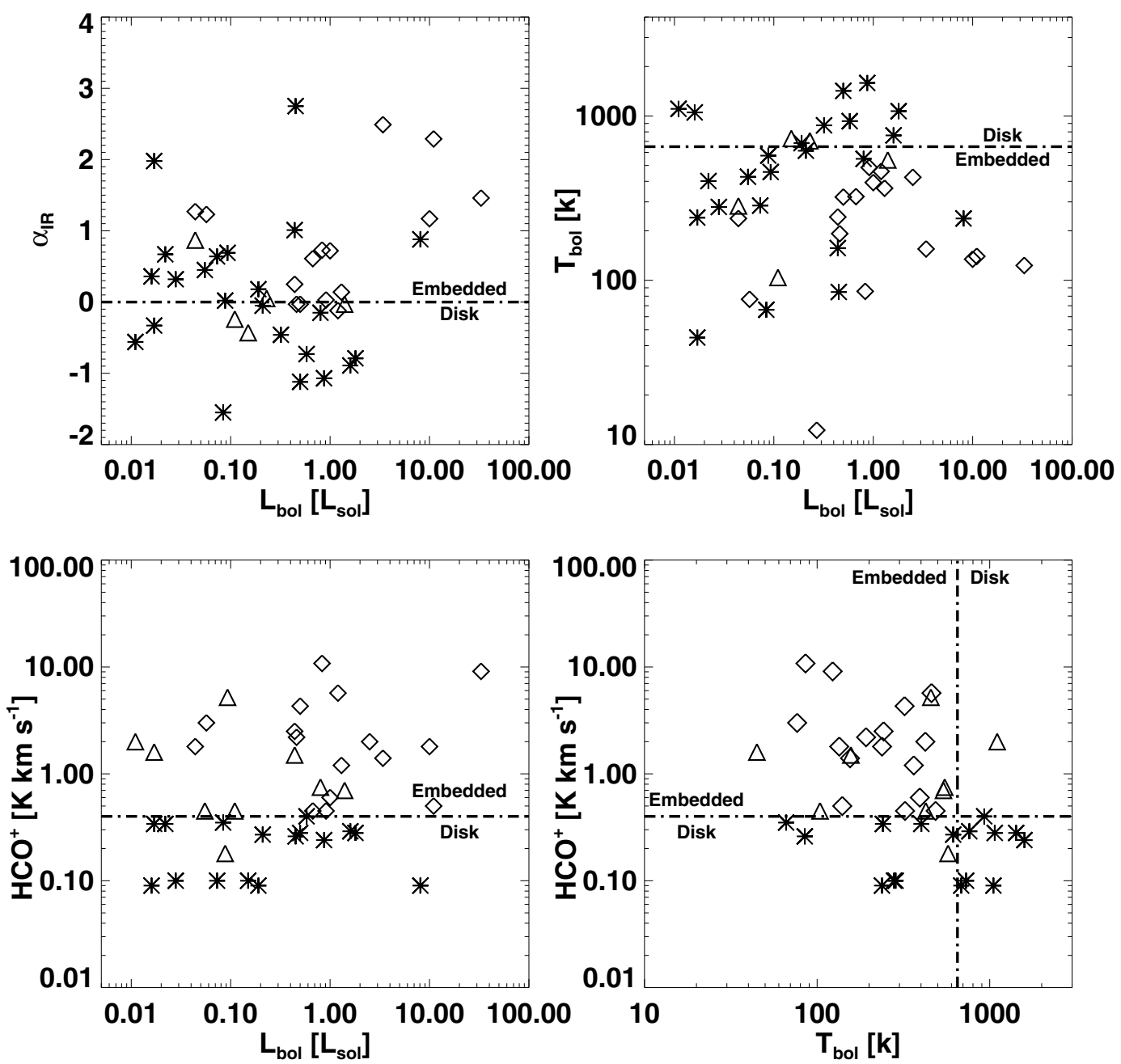

Fig. 18. Classification of sources using the spectral slope (upper left), bolometric temperature (upper right), $\mathrm{HCO}^{+}$intensity (lower left) and $\mathrm{HCO}^{+}$intensity combined with the bolometric temperature (lower right). In the upper two plots, embedded sources are shown with a diamond, disks are shown with a star, and confused sources with a triangle. In the lower two plots, diamonds are again embedded sources, stars represent sources with an upper limit on the $\mathrm{HCO}^{+}$and triangles are sources with no $\mathrm{HCO}^{+}$peak associated with the source. Note that the confused sources and disk sources are both represented with triangles and stars. The traditional limits used for classification, $\alpha_{\mathrm{IR}}>0$ and $T_{\mathrm{bol}}<650 \mathrm{~K}$ as well as our criterion, $\mathrm{HCO}^{+}>0.4 \mathrm{~K} \mathrm{~km} \mathrm{~s}^{-1}$, are shown with dashed lines.

Although most candidate embedded objects are identified by both criteria, several sources are included in their list based on only a single one. Comparison between their list (Table 1 in Jørgensen et al. 2008 limited to L 1688, further referenced as JJ1) and the list in this paper yields the following results:

- Four embedded YSOs with SCUBA fluxes below the cutoff adopted by JJ1 $\left(<0.15 \mathrm{Jy}^{\text {beam }}{ }^{-1}\right.$, the very low envelope masses) are absent from the JJ1 list. Two of these sources (WL6 and IRS 54) are classified by us as late Stage 1 sources with little to no envelope left. The other two embedded YSOs not included in JJ1 are IRS 37 and WL 3.

- Four sources are included in the JJ1 list that have been classified as Stage 2 disks by our method. These are GSS 26, CRBR 2315.8, CRBR 2339.1-2032 and IRS 51. All four sources have associated SCUBA cores and MIPS detections. See the Appendix for the classification reasoning of each of these sources.

\section{Conclusions}

A sample of young stellar objects in L 1688 was analyzed using gas mapping obtained with the new HARP-B heterodyne array receiver in the $\mathrm{HCO}^{+} 4-3$ and $\mathrm{C}^{18} \mathrm{O} 3-2$ lines. Complementary dust maps were obtained from the COMPLETE project, as observed by JCMT-SCUBA, and with SHARC-II on the CSO. The original sample consisted of 45 sources, mostly classified as embedded YSOs or flat-spectrum sources using their spectral slope $\alpha_{\mathrm{IR}}$ in previous work. Of this sample a few sources were recently discovered to be edge-on disks. As a control sample, 4 known disk sources in L 1688 were included. The observations were supplemented by single-pixel observations from APEX, Spitzer-IRS spectroscopy and continuum photometry ranging from $1 \mu \mathrm{m}$ to $1.3 \mathrm{~mm}$, using a variety of space-based and groundbased observatories.

The main conclusions are:

- The concentration of the dense gas, as traced by the $\mathrm{HCO}^{+}$4-3 line mapping, provides an excellent tool to characterize the dense gas in the inner regions of protostellar envelopes. Material in the cold outer envelopes, (edge-on) disks, prestellar cores or cloud material does not emit strongly in $\mathrm{HCO}^{+} 4-3$.

- Most envelopes in L 1688 have low masses, ranging from 0.05 to $0.5 M_{\odot}$. The main accretion phase onto the star has 
already taken place. The only exception is the Stage 0 source VLA 1623 which contains nearly $1 M_{\odot}$.

- A new classification tool based on molecular emission to uniquely identify embedded sources is proposed. Of the total sample, 17 sources were found to be embedded, 5 sources were confused and 23 sources were identified as (edge-on) disks. Due to the foreground layers found through the $\mathrm{C}^{18} \mathrm{O}$, it is concluded that a significant fraction of the sources previously classified as Class I or Flat Spectrum are disks and have been strongly reddened. Combined with the analysis in Jørgensen et al. (2008) all embedded sources in L 1688 were included and characterized down to a mass limit of $0.04 M_{\odot}$ (envelope+disk).

- Four embedded YSOs are found that are in transition from the Stage 1 embedded phase to the Stage 2 T Tauri phase. These sources, IRS 63, IRS 54, WL 6 and WL 17, have little envelope left $\left(M_{\mathrm{env}} \sim 0.05 M_{\odot}\right)$.

- Five sources are so confused by cloud material that identifying them as $\mathrm{T}$ Tauri disks or background sources is not possible with our data. The (lack of) variation and concentration of the molecular emission rules them out as embedded YSOs, however.

- All sources previously identified as disks using traditional methods such as $T_{\text {bol }}$ and $\alpha_{\mathrm{IR}}$ are indeed recovered as disks as are all edge-on disk sources, identified by near-IR mapping (e.g. IRS 46, CRBR 2422.8-3423 and OphE-MM3).

- Spectral line mapping of $\mathrm{C}^{18} \mathrm{O}$ reveals that foreground layers are predominantly present in the Ophiuchus ridge and the Oph-B core. Outside these regions, foreground layers are absent. These foreground layers are responsible for heavy reddening with typical $A_{\mathrm{V}}>20$, confusing classification schemes based on IR colors.

The YSOs in the Ophiuchus clouds show a wide variety of characteristics, ranging from the rare Stage 0 deeply embedded source VLA 1623 and Stage 1 embedded YSOs such as Elias 29, IRS 43 and WL 12 to the embedded YSOs with wimpy envelopes in transition to the T Tauri stage like IRS 63. The results above imply that the molecular emission maps uniquely characterize the envelopes associated with embedded YSOs, while simultaneously identifying disks erroneously classified as embedded YSOs. Such characterization is needed before statistical studies can be used to estimate time scales and star formation efficiencies within L 1688.

One characteristic that has yet to be determined is the ratio of the disk to envelope mass. Interferometric studies with advanced facilities such as the SMA, CARMA or the PdB interferometer will allow the disks to be characterized and, through comparison with continuum single dish studies, the envelope (e.g. Jørgensen et al. 2005; Lommen et al. 2008). Such detailed information will be necessary as a starting point for the interpretation of future observations with Herschel and ALMA of the embedded YSOs in Ophiuchus and other clouds. A central role is envisioned for molecular line data.

Acknowledgements. TvK and astrochemistry at Leiden Observatory are supported by a Spinoza prize and by NWO grant 614.041.004. Remo Tilanus and Jan Wouterloot are thanked for the extensive support on the observing and reduction of HARP-B data and Arno Kockx for carrying out a large part of the HARP-B observations. The support from the APEX and MPIfR staff on the APEX2a data is greatly appreciated. The assistance on the usage of the COMPLETE data from Doug Johnstone and Helen Kirk is recognized. The authors are grateful to the c2d team, especially Mike Dunham (general), Luisa Rebull (MIPS) and Fred Lahuis (IRS), for their help on the Spitzer data and useful discussion.

\section{Appendix: Detailed source description}

GSS 30 and LFAM 1. These sources are both Stage 1 sources with a separation of $15^{\prime \prime}$, but share a common circumbinary envelope on scales up to $40^{\prime \prime}$. Strong $\mathrm{HCO}^{+}$is seen, peaking in between both IR positions. Although outflowing gas was detected, the bulk of the emission is believed to be quiescent.

VLA 1623. $\mathrm{HCO}^{+}$is found to be extended on scales of $30^{\prime \prime}$, larger than that found for most sources. The lack of any emission at IR-wavelengths confirms this to be a Stage 0 source.

WL 12. This source is a good example of a small embedded Stage 1 YSO. It is embedded in a small ridge of material, extending from a north-east to west direction, as can be clearly seen in both dust and $\mathrm{C}^{18} \mathrm{O}$ mapping. This ridge contributes only little to the $\mathrm{HCO}^{+}$emission, which is concentrated at the source position. It is spatially resolved and peaks to $1.4 \mathrm{~K}$. This is much too brigth to be associated with a disk.

LFAM 26. This Stage 1 is located at the tip of the Ophiuchus ridge that extends from LFAM 26 down to IRS 51. See Sect. 5.2 for a discussion on the environment of LFAM 26. The onset of this ridge is clearly seen, extending to the south-east direction, with much less material present in the north-west direction. Both $\mathrm{HCO}^{+}$and $850 \mu \mathrm{m}$ peak at the source position and are bright. Even if the emission originating in the cloud is subtracted from the $\mathrm{HCO}^{+}$emission at the source position, it is too bright to be associated with a disk. This classification is confirmed by the very high $\alpha_{\mathrm{IR}}$ of 1.27 .

WL 17. This is a Stage 1 source, but evolving towards a Stage 2, which we classified "late" Stage 1. The emission of $\mathrm{HCO}^{+}$is too bright to be associated with a disk. Since little to no extended emission is seen at scales of $40^{\prime \prime}$, the source is probably unobscured by cloud material.

Elias 29. This is a well-studied Stage 1 source (Boogert et al. 2002; Lommen et al. 2008). The analysis of $\mathrm{HCO}^{+}$and continuum, both from SCUBA and SHARC-II, confirm this source to be embedded.

IRS 37 and WL 3. The Stage 1 sources IRS 37 and WL 3, separated by $20^{\prime \prime}$ share a common envelope, with both $\mathrm{C}^{18} \mathrm{O}$ and $\mathrm{HCO}^{+}$peaking on both source positions. This envelope is highly non-spherical. The proximity of the two sources is probably responsible for this. Most of the mass is located near IRS 37, although the amount of $\mathrm{HCO}^{+}$emission and concentration of the cores are equal.

WL 6 and GY 256. The weak $\mathrm{HCO}^{+}$seen for these two sources with a separation of $12^{\prime \prime}$ is brighter than that assumed to originate within a disk. In the dust maps, no emission is seen. WL 6 is probably a more evolved Stage 1 source with little envelope mass left. It is possible that GY 256 is a second component in a binary due to the rising spectra between 2 and $24 \mu \mathrm{m}$. However, since the flux from GY 256 at 6 and $8 \mu \mathrm{m}$ is less than at 4.5, it is more likely that GY 256 is a Stage 2 source. GY 256 is classified as confused. 
IRS 43. This is a Stage 1 source, with a well-defined envelope that is bright in $\mathrm{HCO}^{+}$and $\mathrm{C}^{18} \mathrm{O}$. Both the SCUBA and $\mathrm{HCO}^{+}$maps show a slightly elongated envelope with a possible second component about $30^{\prime \prime}$ to the east. This component is relatively much weaker in $\mathrm{HCO}^{+}$than in the dust. However, no IR source is detected at that position. At the position of IRS 43, dense outflowing gas is prominently detected in $\mathrm{HCO}^{+} 4-3$.

IRS 44 and IRS 46. The Stage 2 source IRS 46 was studied by Lahuis et al. (2006) in detail using IRS spectroscopy. Supplementary data from the JCMT confirmed that this source was a disk, reddened by a nearby envelope. The outflowing gas around the Stage 1 source IRS 44 is responsible for the reddening of IRS 46, as seen in Fig. 3.

Elias 32 and 33. The Oph-B2 region dominates the environment around these two Stage 1 sources. At the position of Elias $33, \mathrm{HCO}^{+}$and $850 \mu \mathrm{m}$ continuum clearly peaks. Elias 32 is located in the middle of the Oph-B2 region. A more pronounced SCUBA and a less pronounced $\mathrm{HCO}^{+}$peak are seen within $10^{\prime \prime}$ of Elias 32 and $\alpha_{\mathrm{IR}}$ is in both cases near 0.0 .

IRS 54. Extended emission was found for SCUBA, $\mathrm{HCO}^{+}$and $\mathrm{C}^{18} \mathrm{O}$. In all three cases, the emission is weak. IRS 54 is located outside of the main clouds. Classified as a late Stage 1, this source has accreted most of its envelope mass.

IRAS 16285-2355. This is the only non-late stage 1 source that is located outside of the Ophiuchus ridge, Oph-B2 or Oph-A region. All emission in $\mathrm{HCO}^{+}, \mathrm{C}^{18} \mathrm{O}$ and $850 \mu \mathrm{m}$ continuum is associated with the source in a spherical envelope.

IRS 63. This stage 1 source consists of a large disk with little envelope material. The observed $\mathrm{HCO}^{+}$intensity of $1.2 \mathrm{~K} \mathrm{~km} \mathrm{~s}^{-1}$ confirms that not all envelope material has accreted onto the disk, as suggested by Lommen et al. (2008).

CRBR 2324.1-1619. Due to the location of this source, within $2^{\prime}$ of both VLA 1623 and LFAM 1/GSS 30, and located in between these bright sources, large amounts of material associated with the Oph A core are present, greatly confusing this source. However, the $\mathrm{HCO}^{+}, \mathrm{C}^{18} \mathrm{O}$ or SCUBA emission do not peak at the source position. The spectrum is sharply rising with $\alpha_{\mathrm{IR}}=0.87$. However, this is mainly due to the very high flux seen at $24 \mu \mathrm{m}$.

GY 51. This source was not observed in $\mathrm{HCO}^{+}$. The SCUBA map shows no peak at the position, but bright extended emission in the direction of the Oph-A core. The source is classified as confused, but due to the $\alpha_{\mathrm{IR}}$ of 0.05 , this source is likely a background disk, heavily extincted by the Oph-A cloud material.

WL 19. In the continuum and molecular emission maps, a large amount of material is found, centered $20^{\prime \prime}$ north of WL 19 . This material, probably associated with a pre-stellar core, is responsible for obscuring WL 19. Since the $\alpha_{\mathrm{IR}}=-0.43$ this source is not embedded.
IRS 42. Most of the emission from IRS 42, in both $850 \mu \mathrm{m}$ and $\mathrm{HCO}^{+}$originates within the Ophiuchus ridge, near the core of IRS 43 and the location of CRBR 2422.8-3423.2. This source is classified as confused. However, with a $\alpha_{\mathrm{IR}}=-0.03$ it is more likely that this source is a disk, probably edge-on. The less likely possibility is that this source is a back-ground source.

SSTc2d J162527.6-243648. This source, located far from the main L 1688 regions, is a disk. There is no detected SCUBA or $\mathrm{HCO}^{+}$emission. The rising IR spectrum of 0.36 , combined with the bolometric temperature of $1051 \mathrm{~K}$, is a strong indication that this source is an edge-on Stage 2 source.

GSS 26. Extended SCUBA emission is seen near the source but is likely mostly associated with nearby cloud material. If this cloud emission is subtracted, a small unresolved core remains. The falling $\alpha_{\mathrm{IR}}$ of -0.46 is the main reason that this source was classified as Stage 2.

CRBR 2315.8-1700. Strong $\mathrm{HCO}^{+}$emission and a strongly rising spectrum $\left(\alpha_{\mathrm{IR}}=0.69\right)$ are seen for this source. The $\mathrm{HCO}^{+}$map however suggests that this source is reddened by the large amount of material present in Oph A. An unresolved source is seen in the $850 \mu \mathrm{m}$ data, but that core is not apparent in the $\mathrm{HCO}^{+}$map. No variation was found in the $\mathrm{HCO}^{+} 4-3$ at a level of $0.2 \mathrm{~K} \mathrm{~km} \mathrm{~s}^{-1}$. Thus, this source is likely an disk, in line of sight of the cloud material.

CRBR 2317.3-1925. CRBR 2317.3-1925 was observed with APEX-2a in $\mathrm{HCO}^{+}$and was found to be reasonably strong with a peak temperature of $1.5 \mathrm{~K}$. However, the source does not have a rising spectrum and the SCUBA core seen at the source position is very weak. In addition, the SCUBA emission is extended to the south-east. This source is classified as Stage 2.

VSSG 1. Similar to CRBR 2317.3-1925, VSSG 1 does not have a rising spectrum. Unlike, CRBR 2317.3-1925, however, no $\mathrm{HCO}^{+}$was found with APEX-2a. $850 \mu \mathrm{m}$ emission is detected, but unresolved. The very high $T_{\text {bol }}$, caused by sharply falling spectra long-ward of $24 \mu \mathrm{m}$, confirms this source to be a Stage 2 source, most likely edge-on.

CRBR 2339.1-2032. This Stage 2 source has unresolved emission at $850 \mu \mathrm{m}$. Although $\mathrm{HCO}^{+}$was detected, the contribution is weak, only just above our cut-off for Stage 1, and does not seem associated with the source but with a patch of cloud material about 30" to the north-east of CRBR 2339.1-2032.

WL 2. No $\mathrm{HCO}^{+}$is detected associated with the source. In the dust, a small enhancement is seen at the source position, but it is not brighter than $0.15 \mathrm{Jy} \mathrm{beam}^{-1}$. All $\mathrm{HCO}^{+}$seen in the JCMT map is associated with a small core located between Oph-A and Oph-C. Combined with $\alpha_{\mathrm{IR}}=0.02$ and $T_{\text {bol }}=$ $573 \mathrm{~K}$, the source is classified as a disk, probably edge-on as well as extincted by the cloud material.

GY 224. This source, located south of the Oph ridge, shows no rising spectrum and no $\mathrm{HCO}^{+}$. Since no 850 mum emission 
was found down to the noise limits of the COMPLETE map, this source is concluded to be a Stage 2 .

WL 20S. The emission seen in the SCUBA map does not peak at the position of WL 20S. In the SHARC-II map, a very weak $\left(0.05 \mathrm{Jy} \mathrm{beam}^{-1}\right)$ and unresolved peak is detected. In addition, no $\mathrm{HCO}^{+}$was detected down to the limit adopted for disks. Its location on the Ophiuchus ridge confuses the environment of this source. The very high $\alpha_{\mathrm{IR}}=2.7$ suggests that this source must be an edge-on disk, even if located behind the Ophiuchus ridge.

IRS 48. The disk source IRS 48 is isolated, away from the main cores in Ophiuchus. A weak, unresolved peak is seen in the SCUBA. The absence of any $\mathrm{C}^{18} \mathrm{O}$ and $\mathrm{HCO}^{+}$confirms the classification to be Stage 2.

GY 312. GY 312 is not detected in the dust or gas mapping. In additon, no contribution from large-scale material is seen. Its clearly rising IR spectrum identifies this source as an edge-on Stage 2 source.

IRS 51. IRS 51 is located at the south end of the Ophiuchus ridge. The ridge is clearly seen in SCUBA as well as in $\mathrm{C}^{18} \mathrm{O}$ and $\mathrm{HCO}^{+}$maps around IRS 51. Although a clear peak is seen at IRS 51 in the dust map, no peak was seen in the molecular line mapping at the position of IRS 51. It is concluded that IRS 51 is a Stage 2 source with strong disk emission, reddened by the Ophiuchus ridge.

SSTc2d J162741.6-244645. This stage 2 source, located south of the Ophiuchus ridge, was not detected in either $\mathrm{HCO}^{+}$or SCUBA. The rising spectrum suggests that this source must be edge-on since no cloud material is nearby to redden the source.

SSTc2d J162748.2-244225. For this source, located east of the southern tip of the Ophiuchus ridge, no SCUBA or $\mathrm{HCO}^{+}$emission was found. The very high $\alpha_{\mathrm{IR}}$ of 1.98 suggests that this source must be an edge-on disk.

SSTc2d J162857.9-244055. This source is located between the L 1688 and L 1689 regions. The combination of a steeply rising spectrum and an absence of $\mathrm{HCO}^{+}$and $850 \mu \mathrm{m}$ continuum indicates that this source is a edge-on Stage 2 source.

Haro 1-4. Located north of the main Ophiuchus clouds, this source has no $\mathrm{HCO}^{+}$or $850 \mu \mathrm{m}$ associated with it and clearly is a disk.

DoAr 25. Although a bright peak is seen in the $850 \mu \mathrm{m}$ map, the $\alpha_{\mathrm{IR}}$ of -1.12 clearly confirms this to be a disk source.

OphE MM3. This source, located within the Ophiuchus ridge, was first discovered in the mm-regime by Motte et al. (1998). Both the $850 \mu \mathrm{m}$ continuum and $\mathrm{HCO}^{+}$show little material associated with an envelope at this source. Near-IR observations (Brandner et al. 2000) confirm it to be an edge-on disk.
SR 21. This source has a very high bolometric temperature of $1070 \mathrm{~K}$. Although a clear signal is seen in the $850 \mu \mathrm{m}$ data, no emission is seen in $\mathrm{HCO}^{+}$. It has been confirmed as a cold disk with a large inner hole by Brown et al. (2007).

CRBR 2422.8-3423. CRBR 2422.8-3423 is located along the Ophiuchus ridge. From near-IR imaging (Brandner et al. 2000; Pontoppidan et al. 2005) it is known that CRBR 2422.8-3423 is an edge-on disk and located in line of sight of the IRS 43 envelope. Even at far-IR wavelengths, emission from the Ophiuchus ridge rather than the source dominates. $\mathrm{HCO}^{+}$mapping confirms this.

SR 9. This source is not detected at $850 \mu \mathrm{m}$. Combined with $\alpha_{\mathrm{IR}}<-1$, it is concluded that SR 9 is a Stage 2 source.

2MASS 16282. Although 2MASS 16282 has a very low bolometric temperature of $66 \mathrm{~K}$, the $\alpha_{\mathrm{IR}}<-1$ identifies this source as a disk.

\section{References}

Adams, F. C., Lada, C. J., \& Shu, F. H. 1987, ApJ, 312, 788

Alexander, R. D., Casali, M. M., André, P., Persi, P., \& Eiroa, C. 2003, A\&A, 401,613

André, P., \& Montmerle, T. 1994, ApJ, 420, 837

André, P., Ward-Thompson, D., \& Barsony, M. 1993, ApJ, 406, 122

Andrews, S. M., \& Williams, J. P. 2007, ApJ, 671, 1800

Barsony, M., Kenyon, S. J., Lada, E. A., \& Teuben, P. J. 1997, ApJS, 112, 109

Barsony, M., Ressler, M. E., \& Marsh, K. A. 2005, ApJ, 630, 381

Blake, G. A., van Dishoek, E. F., Jansen, D. J., Groesbeck, T. D., \& Mundy, L. G. 1994, ApJ, 428, 680

Blake, G. A., Sandell, G., van Dishoeck, E. F., et al. 1995, ApJ, 441, 689

Bontemps, S., André, P., Kaas, A. A., et al. 2001, A\&A, 372, 173

Boogert, A. C. A., Tielens, A. G. G. M., Ceccarelli, C., et al. 2000, A\&A, 360, 683

Boogert, A. C. A., Hogerheijde, M. R., Ceccarelli, C., et al. 2002, ApJ, 570, 708

Boogert, A., Pontoppidan, K., Knez, C., et al. 2008, ArXiv e-prints, 801

Brandner, W., Sheppard, S., Zinnecker, H., et al. 2000, A\&A, 364, L13

Brown, J. M., Blake, G. A., Dullemond, C. P., et al. 2007, ApJ, 664, L107

Cambrésy, L. 1999, A\&A, 345, 965

Chapman, N., Mundy, L., Lai, S.-P., \& Evans, N. 2008, ApJ, in press

Comeron, F., Rieke, G. H., Burrows, A., \& Rieke, M. J. 1993, ApJ, 416, 185

Crapsi, A., van Dishoeck, E. F., Hogerheijde, M. R., Pontoppidan, K. M., \& Dullemond, C. P. 2008, ArXiv e-prints, 801

Di Francesco, J., Johnstone, D., Kirk, H., MacKenzie, T., \& Ledwosinska, E. 2008, ApJS, 175, 277

Dickman, R. L., \& Herbst, W. 1990, ApJ, 357, 531

Dowell, C. D., Allen, C. A., Babu, R. S., et al. 2003, SPIE Conf., 4855, 73

Dunham, M. M., Crapsi, A., Evans, II, N. J., et al. 2008, ArXiv e-prints, 806

Elias, J. H. 1978, ApJ, 224, 453

Enoch, M. L., Evans, N. J., Sargent, A. I., \& Glenn, J. 2008, ApJ, submitted

Evans, N. J. 1999, ARA\&A, 37, 311

Evans, N. J., Allen, L. E., Blake, G. A., et al. 2003, PASP, 115, 965

Evans, N. J., Harvey, P. M., Dunham, M. M., et al. 2007, c2d delivery document

Evans, II, N. J., Dunham, M. M., Jørgensen, J. K., et al. 2009, ApJ, in press

Greene, T. P., \& Meyer, M. R. 1995, ApJ, 450, 233

Greene, T. P., Wilking, B. A., Andre, P., Young, E. T., \& Lada, C. J. 1994, ApJ, 434, 614

Hogerheijde, M. R., van Dishoeck, E. F., Blake, G. A., \& van Langevelde, H. J. 1997, ApJ, 489, 293

Jensen, E. L. N., Mathieu, R. D., \& Fuller, G. A. 1996, ApJ, 458, 312

Johnstone, D., Wilson, C. D., Moriarty-Schieven, G., et al. 2000, ApJ, 545, 327

Johnstone, D., Fich, M., Mitchell, G. F., \& Moriarty-Schieven, G. 2001, ApJ, 559,307

Jørgensen, J. K. 2004, A\&A, 424, 589

Jørgensen, J. K., Schöier, F. L., \& van Dishoeck, E. F. 2002, A\&A, 389, 908 Jørgensen, J. K., Schöier, F. L., \& van Dishoeck, E. F. 2005, A\&A, 437, 501 
Jørgensen, J. K., Johnstone, D., van Dishoeck, E. F., \& Doty, S. D. 2006, A\&A, 449, 609

Jørgensen, J. K., Bourke, T. L., Myers, P. C., et al. 2007, ApJ, 659, 479

Jørgensen, J. K., Johnstone, D., Kirk, H., et al. 2008, ApJ, in press

Kirk, H., Johnstone, D., \& Di Francesco, J. 2006, ApJ, 646, 1009

Knude, J., \& Høg, E. 1998, A\&A, 338, 897

Lada, C. J., \& Wilking, B. A. 1984, ApJ, 287, 610

Lahuis, F., van Dishoeck, E. F., Boogert, A. C. A., et al. 2006, ApJ, 636, L145

Liseau, R., White, G. J., Larsson, B., et al. 1999, A\&A, 344, 342

Loinard, L., Torres, R. M., Mioduszewski, A. J., \& Rodríguez, L. F. 2008, ApJ, 675, L29

Lommen, D., Jørgensen, J. K., van Dishoeck, E. F., \& Crapsi, A. 2008, A\&A, 481,141

Looney, L. W., Mundy, L. G., \& Welch, W. J. 2000, ApJ, 529, 477

Loren, R. B. 1989, ApJ, 338, 902

Loren, R. B., Wootten, A., \& Wilking, B. A. 1990, ApJ, 365, 269

Luhman, K. L., \& Rieke, G. H. 1999, ApJ, 525, 440

Maret, S., Ceccarelli, C., Caux, E., et al. 2004, A\&A, 416, 577

Maret, S., Ceccarelli, C., Tielens, A. G. G. M., et al. 2005, A\&A, 442, 527

Mezger, P. G., Sievers, A., Zylka, R., et al. 1992, A\&A, 265, 743

Motte, F., Andre, P., \& Neri, R. 1998, A\&A, 336, 150

Myers, P. C., \& Ladd, E. F. 1993, ApJ, 413, L47

Nuernberger, D., Brandner, W., Yorke, H. W., \& Zinnecker, H. 1998, A\&A, 330, 549

Ossenkopf, V., \& Henning, T. 1994, A\&A, 291, 943

Padgett, D. L., Rebull, L. M., Stapelfeldt, K. R., et al. 2008, ApJ, 672, 1013

Pontoppidan, K. M., Dullemond, C. P., van Dishoeck, E. F., et al. 2005, ApJ, 622,463

Pontoppidan, K. M., Fraser, H. J., Dartois, E., et al. 2003, A\&A, 408, 981

Rachford, B. L., Snow, T. P., Tumlinson, J., et al. 2002, ApJ, 577, 221
Ridge, N. A., Di Francesco, J., Kirk, H., et al. 2006, AJ, 131, 2921

Robitaille, T. P., Whitney, B. A., Indebetouw, R., Wood, K., \& Denzmore, P. 2006, ApJS, 167, 256

Robitaille, T. P., Whitney, B. A., Indebetouw, R., \& Wood, K. 2007, ApJS, 169, 328

Schöier, F. L., Jørgensen, J. K., van Dishoeck, E. F., \& Blake, G. A. 2002, A\&A, 390,1001

Schöier, F. L., Jørgensen, J. K., van Dishoeck, E. F., \& Blake, G. A. 2004, A\&A, 418, 185

Shirley, Y. L., Evans, N. J., Rawlings, J. M. C., \& Gregersen, E. M. 2000, ApJS, 131,249

Smith, H., Hills, R. E., Withington, S., et al. 2003, Presented at the Society of Photo-Optical Instrumentation Engineers (SPIE) Conference, 4855, 338

Stanke, T., Smith, M. D., Gredel, R., \& Khanzadyan, T. 2006, A\&A, 447, 609

Thi, W.-F., van Zadelhoff, G.-J., \& van Dishoeck, E. F. 2004, A\&A, 425, 955

van der Tak, F. F. S., Black, J. H., Schöier, F. L., Jansen, D. J., \& van Dishoeck, E. F. 2007, A\&A, 468, 627

Walawender, J., Bally, J., Kirk, H., \& Johnstone, D. 2005, AJ, 130, 1795

Whitney, B. A., Wood, K., Bjorkman, J. E., \& Cohen, M. 2003a, ApJ, 598, 1079

Whitney, B. A., Wood, K., Bjorkman, J. E., \& Wolff, M. J. 2003b, ApJ, 591, 1049

Wilking, B. A., \& Lada, C. J. 1983, ApJ, 274, 698

Wilking, B. A., Lada, C. J., \& Young, E. T. 1989, ApJ, 340, 823

Wilson, T. L., \& Rood, R. 1994, ARA\&A, 32, 191

Wootten, A. 1989, ApJ, 337, 858

Young, C. H., Shirley, Y. L., Evans, II, N. J., \& Rawlings, J. M. C. 2003, ApJS, 145,111

Young, C. H., Jørgensen, J. K., Shirley, Y. L., et al. 2004, ApJS, 154, 396

Young, K. E., Enoch, M. L., Evans, II, N. J., et al. 2006, ApJ, 644, 326 